\title{
Massless relativistic wave equations and quantum field theory
}

\author{
FERnANDo Lledó \\ Institute for Pure and Applied Mathematics, \\ RWTH-Aachen, Templergraben 55, \\ D-52056 Aachen, Germany. \\ lledo@iram.rwth-aachen.de
}

January 18, 2010

Dedicated to Rudolf Haag on his 80th birthday.

\begin{abstract}
We give a simple and direct construction of a massless quantum field with arbitrary discrete helicity that satisfies Wightman axioms and the corresponding relativistic wave equation in the distributional sense. We underline the mathematical differences to massive models. The construction is based on the notion of massless free net (cf. Section 3) and the detailed analysis of covariant and massless canonical (Wigner) representations of the Poincaré group. A characteristic feature of massless models with nontrivial helicity is the fact that the fibre degrees of freedom of the covariant and canonical representations do not coincide. We use massless relativistic wave equations as constraint equations reducing the fibre degrees of freedom of the covariant representation. They are characterised by invariant (and in contrast with the massive case non reducing) one-dimensional projections. The definition of one-particle Hilbert space structure that specifies the quantum field uses distinguished elements of the intertwiner space between $\mathcal{E}(2)$ (the two-fold cover of the 2-dimensional Euclidean group) and $\overline{\mathcal{E}(2)}$.

We conclude with a brief comparison between the free nets constructed in Section 3 and a recent alternative construction that uses the notion of modular localisation.
\end{abstract}

\section{Introduction}

The transformation character of a quantum field involves typically two different types of representations of the corresponding spacetime symmetry group. The first one is a so-called covariant representation which acts reducibly on the test function space of the quantum field. The second one is a unitary and irreducible representation, which is called canonical (and in certain cases also Wigner representation), and which acts on the one-particle Hilbert space associated to the quantum field (see e.g. 75. Section 2]). In the context of non-scalar free quantum field theory on Minkowski space, one chooses as test function space $\mathcal{H}$-valued Schwartz functions, where $\mathcal{H}$ is a fixed finite-dimensional Hilbert space with $\operatorname{dim} \mathcal{H} \geq 2$. Moreover, in order to describe massless models with discrete helicity, the corresponding space carrying the Wigner representations is a $\mathbb{C}$-valued $L^{2}$ space over the positive light cone. (In contrast with it, massive theories use $\mathcal{H}$-valued $L^{2}$ functions over the positive mass shell.) The fact that there is a difference between the dimensions of the image Hilbert spaces (fibres) of the preceding two function spaces ( $\mathcal{H}$ vs. $\mathbb{C}$ valued functions), forces one to introduce some additional set of constraints in the construction 
of massless models with non-trivial helicity. These constraints guarantee for example that one can embed the test functions into the space carrying the corresponding massless Wigner representation. Physically they express the fact that for massless particles the helicity is parallel to the momentum in all Lorentz frames. In previous papers we have shown that this reduction in the fibre space can be performed in two different ways (see Remark 2.11 below). In this paper we will develop a third point of view in order to explain the reduction, where massless relativistic wave equations will play an essential role. Indeed, one may consider massless relativistic wave equations as constraint equations that reduce the fibre degrees of freedom.

Free massless quantum fields with discrete helicity were constructed by Weinberg in [72]. The necessary reduction of degrees of freedom mentioned above has been done in this reference as follows: to define a massless quantum field of helicity $j, 2 j \in \mathbb{Z}$, one usually constructs first a $2 j+1$-component free quantum field. This initial step is a clear reminiscence of massive theories and in fact the unnecessary components are ruled out afterwards by imposing on the quantum field itself a first order constraint equation. This construction procedure has been reproduced almost unchanged several times in the literature (cf. [74, 40, 73]). In the present paper we propose an alternative and direct construction of a free massless quantum field with arbitrary discrete helicity which satisfies the corresponding massless relativistic wave equation in a distributional sense. We will underline the mathematical structures characteristic to massless theories that appear in the group theoretical as well as in the quantum field theoretical context. The construction is naturally suggested by a detailed mathematical analysis of the covariant and canonical representations and, in fact, the reduction of the degrees of freedom can be encoded in suitable one-dimensional invariant projections. In this way the covariant transformation character of the quantum fields becomes completely transparent. They will also satisfy the corresponding Wightman axioms.

The main aspects of this paper may be summarised in the following three items:

(i) We analyse from a mathematical point of view the role of classical massless relativistic wave equations in the context of induced representations of the Poincaré group. We show that these equations are characterised by certain invariant (but not reducing) one-dimensional projections. This analysis extends a systematic study of Niederer and O'Rafeartaigh (cf. 54]) concerning massive relativistic wave equations. We will point out the differences w.r.t. the massive case (see Section 2).

(ii) We will give an alternative construction of massless free nets using as the essential element an embedding $\mathfrak{I}$ from the test function space to the space of solution of the corresponding massless relativistic wave equation. This embedding intertwines the covariant and canonical representations mentioned above and therefore we can partly interpret the free net construction in the group theoretical context (see Section 31).

(iii) We can finally reinterpret the previous embedding $\mathfrak{I}$ as a one-particle Hilbert space structure and this allows to give a new construction procedure for massless quantum fields with nontrivial helicity. These fields will satisfy directly the relativistic wave equation in the distributional sense as well as the Wightman axioms. We will finally mention some further properties of these fields like e.g. the conformal covariance (see Section 4).

In order to describe induced representations we will consider in the following section the elegant fibre bundle language. In particular the crucial covariant and canonical representations of the Poincaré group can be described as two different special cases in this framework. (In this context we can even describe the representations of the conformal group that restrict to the massless Wigner representations with discrete helicity (cf. 48])). From a mathematical point of view, the reason for the need of reducing the fibre degrees of freedom mentioned at the beginning of the introduction lies in the following facts: on the one hand, the canonical or Wigner 
representations describing massless particles with discrete helicity are induced from non-faithful, one-dimensional representations of the corresponding little group $\mathcal{E}(2)$ (the two-fold cover of the 2-dimensional Euclidean group). On the other hand, the covariant representations are induced from at least two-dimensional irreducible representation of the little group $\mathrm{SL}(2, \mathbb{C})$ which do not restrict (for non-scalar models) to the inducing representation of the canonical representation when considering $\mathcal{E}(2)$ as a subgroup of $\mathrm{SL}(2, \mathbb{C})$. In its turn the use of non-faithful representations is due to the fact that the massless little group $\mathcal{E}(2)$ is non-compact, solvable and has a semi-direct product structure. (Recall that, in contrast with the previous attributes, the massive little group $\mathrm{SU}(2)$ satisfies the complementary properties of being compact and simple.) In the context of massless canonical representations massless relativistic wave equations will naturally appear as constraints performing the mentioned reduction and indeed we may associate with them invariant (but in contrast to massive equations non reducing) projections. It becomes clear that massless relativistic wave equations have a different character than massive ones and are in a certain sense unavoidable for nonscalar models, even if one does not consider discrete transformations of Minkowski space.

We will also use in the present paper spinor fields. They can be roughly seen as being "square roots" of null vector fields (cf. [70]) and massless relativistic wave equations can be simply and systematically expressed as differential equations involving these type of fields (cf. 55, 56]). The spinorial language is also particularly well adapted to the general group theoretical framework mentioned before. In the following section we will in addition work out explicitly the Weyl equation as well as Maxwell's equations (in terms of the field strength, $\mathfrak{F}$-eq. for short). These two concrete examples describing models with helicity $\frac{1}{2}$ and 1 will be the base for the construction of massless fermionic and bosonic free nets/fields with nontrivial helicity. For completeness we will include in our group theoretical context the discussion of the classical Maxwell Equations in terms of the vector potential field ( $\mathfrak{A}$-eq. for short). For a detailed treatment of quantum electromagnetism in terms of the vector potential (including constraints) we refer to [32. An alternative and systematic analysis of relativistic particles in the context of geometric quantisation is given in [23, 24]. There are other approaches to classical massless relativistic wave equations with different degrees of mathematical rigor, e.g. [76, Chapter II], 69, Chapter 9] or [44, 29, 52, 49, 7, 26, 25.

Concerning item (ii) mentioned previously, we define and prove in Section 3 the main properties of a massless free net. Recall that free nets, as considered in [12, 47] (see also [14]), are the result of a direct and natural way of constructing nets of abstract $\mathrm{C}^{*}$-algebras indexed by open and bounded regions in Minkowski space. They also satisfy the axioms of local quantum physics. The construction is based on group theoretical arguments (in particular on the covariant and canonical representations of the Poincaré group mentioned before) and standard theory of CARand CCR-algebras [5, 51. No representation of the $\mathrm{C}^{*}$-algebra is used and no quantum fields are initially needed. This agrees with the point of view in local quantum physics that the abstract algebraic structure should be a primary definition of the theory and the corresponding Hilbert space representation a secondary [21, Section 4]. Nevertheless, the free nets will afterwards canonically suggest the construction procedure for massless quantum fields. Therefore the massless free net construction presented here justifies the picture that free nets are the counterpart of free fields at the abstract $\mathrm{C}^{*}$-algebraic level. In addition, the notion of free nets (cf. Part (ii) of Definition 3.3) contains some aspects of Segal's concept of quantisation for bosonic systems (cf. 61, p. 750], [8, p. 106]). Through the requirements on the embedding $\mathfrak{I}$ we incorporate to this program the axioms of local quantum physics. Note, however, that since Haag-Kastler axioms are stated in terms of abstract $\mathrm{C}^{*}$-algebras, we do not initially require (in contrast with Segal's approach) that the abstract CCR-algebra is represented in any Hilbert space nor it is necessary to specify any regular state. This point of view is relevant when constraints are present (cf. [31]). Furthermore, the construction presented in Section 3, is in a certain sense complementary to the construction 
considered in 48, but still will produce isomorphic nets. Instead of using semidefinite sesquilinear forms and the related factor spaces as in [48] we will use in the present paper explicitly the space of solutions of the corresponding massless relativistic wave equation and therefore we need to introduce a different embedding $\mathfrak{I}$ for characterising the net. Further in [48] we focused on the covariance of the massless free net under the Poincaré and conformal group. Here we prove the rest of the properties satisfied by the free net (e.g. additivity, causality etc.).

The previous construction of a free net and in particular the group theoretical interpretation of the embedding $\mathfrak{I}$ particularly pays off in the new construction procedure of massless quantum fields as well as in the verification of the corresponding axioms. Indeed, for the construction procedure of the massless quantum field (cf. item (iii) above) we need to reinterpret $\mathfrak{I}$ as a one-particle Hilbert space structure. In this context we show the continuity of $\mathfrak{I}$ w.r.t. the corresponding Schwartz space and Hilbert space topologies. Moreover, these fields will directly satisfy

the (constraint) massless relativistic wave equations. E.g. in the helicity $\frac{1}{2}$ case the quantum field satisfies in the distributional sense

$$
\partial^{C C^{\prime}} \phi\left(f_{C}\right)=0
$$

where $f$ is the corresponding vector-valued Schwartz function. The construction presented here is considerably simpler than what is usually done in QFT. In order to define the fields we will use inducing representations of the type $D^{\left(\frac{n}{2}, 0\right)}$ and $D^{\left(0, \frac{n}{2}\right)}$ since these are enough to construct massless quantum fields with helicity $\pm \frac{n}{2}$ (see e.g. [53]).

We conclude this paper commenting on the relations of the free net construction in Section 3 with the construction given in [20] which is based on the notion of modular localisation (see also [27]).

\section{Representations of the Poincaré group and relativistic wave equations}

In the present section we will summarise some results concerning the theory of induced representations in the context of fibre bundles. For details and further generalisations we refer to [6. 64, 65] and [71, Section 5.1]. We will specify these structures in the example of the Poincaré group to introduce so-called covariant and massive/massless canonical representations. In this group theoretical context we will consider massive and massless relativistic wave equations and analyse their different character. For a study of the conformal group (in 4-dimensions) in this frame and for a proof of the extension of the massless canonical representations with discrete helicity to a corresponding representation of the conformal group see [48] and references cited therein.

Let $\mathcal{G}$ be a Lie group that acts differentiably and transitively on a $\mathrm{C}^{\infty}$-manifold $M$. For $u_{0} \in M$ denote by $\mathcal{K}_{0}:=\left\{g \in \mathcal{G} \mid g u_{0}=u_{0}\right\}$ the corresponding isotropy group w.r.t. this action. Then, from [38, Theorem II.3.2 and Proposition II.4.3] we have that $g \mathcal{K}_{0} \mapsto g u_{0}$ characterises the diffeomorphism

$$
\mathcal{G} / \mathcal{K}_{0} \cong \mathbb{D}:=\left\{g u_{0} \mid g \in \mathcal{G}\right\} .
$$

In this context we may consider the following principal $\mathcal{K}_{0}$-bundle,

$$
\mathcal{B}_{1}:=\left(\mathcal{G}, \operatorname{pr}_{1}, \mathbb{D}\right) \text {. }
$$

$\operatorname{pr}_{1}: \mathcal{G} \rightarrow \mathbb{D}$ denotes the canonical projection onto the base space $\mathbb{D}$. Given a representation $\tau: \mathcal{K}_{0} \rightarrow \mathrm{GL}(\mathcal{H})$ on the finite-dimensional Hilbert space $\mathcal{H}$, one can construct the associated vector bundle

$$
\mathcal{B}_{2}(\tau):=\left(\mathcal{G} \times_{\mathcal{K}_{0}} \mathcal{H}, \mathrm{pr}_{2}, \mathbb{D}\right)
$$


(see [42, Section I.5] for further details). The action of $\mathcal{G}$ on $M$ specifies the following further actions on $\mathbb{D}$ and on $\mathcal{G} \times_{\mathcal{K}_{0}} \mathcal{H}$ : for $g, g_{0} \in \mathcal{G}, v \in \mathcal{H}$, put

$$
\left.\begin{array}{rlrl}
\mathcal{G} \times \mathbb{D} & \longrightarrow \mathbb{D}, & g_{0} \operatorname{pr}_{1}(g):=\operatorname{pr}_{1}\left(g_{0} g\right) \\
\mathcal{G} \times\left(\mathcal{G} \times \mathcal{K}_{0} \mathcal{H}\right) & \longrightarrow \mathcal{G} \times_{\mathcal{K}_{0}} \mathcal{H}, & g_{0}[g, v]:=\left[g_{0} g, v\right]
\end{array}\right\}
$$

where $[g, v]=\left[g k^{-1}, \tau(k) v\right], k \in \mathcal{K}_{0}$, denotes the equivalence class characterising a point in the total space of the associated bundle. Finally we define the (from $\tau$ ) induced representation of $\mathcal{G}$ on the vector space of sections of the vector bundle $\mathcal{B}_{2}$, which we denote by $\Gamma\left(\mathcal{G} \times_{\mathcal{K}_{0}} \mathcal{H}\right)$ : let $\psi$ be such a section and for $g \in \mathcal{G}, p \in \mathbb{D}$ :

$$
(T(g) \psi)(p):=g \psi\left(g^{-1} p\right) .
$$

2.1 Remark We will now present a way of rewriting the preceding induced representation in (for physicists more usual) terms of vector-valued functions. Indeed, choose a fixed (not necessarily continuous) section $s: \mathbb{D} \rightarrow \mathcal{G}$ of the principal $\mathcal{K}_{0}$-bundle $\mathcal{B}_{1}$. Now for $\psi \in \Gamma\left(\mathcal{G} \times \mathcal{K}_{0} \mathcal{H}\right)$ we put $\psi(p)=[s(p), \varphi(p)], p \in \mathbb{D}$, for a suitable function $\varphi: \mathbb{D} \rightarrow \mathcal{H}$ and we may rewrite the induced representation as

$$
(T(g) \varphi)(p)=\tau\left(s(p)^{-1} g s\left(g^{-1} p\right)\right) \varphi\left(g^{-1} p\right),
$$

where it can be easily seen that $s(p)^{-1} g s\left(g^{-1} p\right) \in \mathcal{K}_{0}$.

Note that till now we have not completely specified the induced representation $T$. In fact, we have to fix the structure of the representation space $\Gamma\left(\mathcal{G} \times{ }_{\mathcal{K}_{0}} \mathcal{H}\right.$ ) (or of the set of $\mathcal{H}$-valued functions). This must be done separately in the three concrete situations considered below: covariant representations as well as massive and massless canonical representations. In these cases we have to specify completely the structure of the corresponding representation spaces. We will also give regularity conditions on the fixed section $s$ of the principal bundle $\mathcal{B}_{1}$ considered before. Typically we will work with fixed Borel or continuous sections of the corresponding principal bundles.

\subsection{The Poincaré group:}

We will apply next the general scheme of induced representations presented above to specify the so-called covariant and canonical representations of the Poincaré group. These will play a fundamental role in the definition of the free net in the next section. For additional results concerning induced representations and for the physical interpretation of the canonical (or Wigner) representations we refer to [9, 10, 50, 64, 77] as well as [45, Section 2.1] and 68, Chapters 2 and $3]$.

Covariant representations: In the general analysis considered above let $\mathcal{G}:=\mathrm{SL}(2, \mathbb{C}) \ltimes \mathbb{R}^{4}=$ $\widetilde{\mathcal{P}_{+}^{\uparrow}}$ be the universal covering of the proper orthocronous component of the Poincaré group. It acts on $M:=\mathbb{R}^{4}$ in the usual way $(A, a) x:=\Lambda_{A} x+a,(A, a) \in \mathrm{SL}(2, \mathbb{C}) \ltimes \mathbb{R}^{4}, x \in \mathbb{R}^{4}$, where $\Lambda_{A}$ is the Lorentz transformation associated to $\pm A \in \mathrm{SL}(2, \mathbb{C})$ which describes the action of $\mathrm{SL}(2, \mathbb{C})$ on $\mathbb{R}^{4}$ in the semi-direct product. Putting now $u_{0}:=0$ gives $\mathcal{K}_{0}=\mathrm{SL}(2, \mathbb{C}) \ltimes\{0\}$, $\mathcal{G} /(\mathrm{SL}(2, \mathbb{C}) \ltimes\{0\}) \cong \mathbb{R}^{4}$, and the principal $\mathrm{SL}(2, \mathbb{C})$-bundle is in this case $\mathcal{B}_{1}:=\left(\mathcal{G}, \mathrm{pr}_{1}, \mathbb{R}^{4}\right)$. As inducing representation we use the finite-dimensional irreducible representations of $\mathrm{SL}(2, \mathbb{C})$ acting on the spinor space $\mathcal{H}^{\left(\frac{j}{2}, \frac{k}{2}\right)}:=\operatorname{Sym}\left(\stackrel{j}{\otimes} \mathbb{C}^{2}\right) \otimes \operatorname{Sym}\left(\stackrel{k}{\otimes} \mathbb{C}^{2}\right)$ (cf. [66] $)$ : i.e. $\tau^{(\text {cov })}(A, 0):=$ $D^{\left(\frac{j}{2}, \frac{k}{2}\right)}(A)=(\stackrel{j}{\otimes} A) \otimes(\stackrel{k}{\otimes} \bar{A}),(A, 0) \in \mathrm{SL}(2, \mathbb{C}) \ltimes\{0\}$. From this we have (if no confusion arises we will omit in the following the index $\left(\frac{j}{2}, \frac{k}{2}\right)$ in $D(\cdot)$ and in $\left.\mathcal{H}\right)$,

$$
\mathcal{B}_{2}\left(\tau^{(\mathrm{cov})}\right):=\left(\mathcal{G} \times \times_{\mathrm{SL}(2, \mathbb{C})} \mathcal{H}, \mathrm{pr}_{2}, \mathbb{R}^{4}\right)
$$


Recalling Remark 2.1] we specify a global continuous section $s$ of $\mathcal{B}_{1}$ (i.e. $\mathcal{B}_{1}$ is a trivial bundle):

$$
s: \mathbb{R}^{4} \longrightarrow \mathcal{G}, \quad s(x):=(\mathbb{1}, x) \in \mathrm{SL}(2, \mathbb{C}) \ltimes \mathbb{R}^{4}=\mathcal{G} .
$$

Note that since $\tau^{\text {(cov) }}$ is not a unitary representation and since we want to relate the following socalled covariant representation with the irreducible and unitary canonical ones presented below, it is enough to define $T$ on the space of $\mathcal{H}$-valued Schwartz functions $\mathcal{S}\left(\mathbb{R}^{4}, \mathcal{H}\right)$

$$
(T(g) f)(x):=D(A) f\left(\Lambda_{A}^{-1}(x-a)\right), \quad f \in \mathcal{S}\left(\mathbb{R}^{4}, \mathcal{H}\right),
$$

where we have used that $s(x)^{-1}(A, a) s\left((A, a)^{-1} x\right)=(A, 0),(A, a) \in \mathcal{G}$. $T$ is an algebraically reducible representation even if the inducing representation $\tau^{(\mathrm{cov})}$ is irreducible.

2.2 Remark We will show later that the covariant representation above is related with the covariant transformation character of quantum fields. Thus a further reason for considering this representation space is the fact that in the heuristic picture we want to smear quantum fields with test functions in $\mathcal{S}\left(\mathbb{R}^{4}, \mathcal{H}\right)$.

Canonical representations: Next we will consider unitary and irreducible canonical representations of $\mathcal{P}_{+}^{\uparrow}$ and, in particular, we will specify the massive and those massless ones with discrete helicity. We will also state the mathematical differences between these two types of representations. We will mainly apply here Mackey's theory of induced representations of regular semi-direct products, where each subgroup is locally compact and one of them abelian [50, 64, 10. (However, see also Remark 2.18 (b)).

First note that in the general context of the beginning of this section if $\tau$ is a unitary representation of $\mathcal{K}_{0}$ on $\mathcal{H}$, then $\Gamma\left(\mathcal{G} \times_{\mathcal{K}_{0}} \mathcal{H}\right)$ turns naturally into a Hilbert space. Indeed, the fibres $\operatorname{pr}_{2}^{-1}(p), p \in \mathbb{D}$, inherit a unique (modulo unitary equivalence) Hilbert space structure from $\mathcal{H}$. Assume further that $\mathbb{D}$ allows a $\mathcal{G}$-invariant measure $\mu$. (The following construction goes also through with little modifications if we only require the existence on $\mathbb{D}$ of a quasi-invariant measure w.r.t. $\mathcal{G}$.) Then $\Gamma\left(\mathcal{G} \times \mathcal{K}_{0} \mathcal{H}\right)$ is the Hilbert space of all Borel sections $\psi$ of $\mathcal{B}_{2}(\tau)$ that satisfy,

$$
\langle\psi, \psi\rangle=\int_{\mathbb{D}}\langle\psi(p), \psi(p)\rangle_{p} \mu(\mathrm{d} p)<\infty,
$$

where $\langle\cdot, \cdot\rangle_{p}$ denotes the scalar product on the Hilbert space $\operatorname{pr}_{2}^{-1}(p), p \in \mathbb{D}$, and the induced representation given in Eq. (44) is unitary on it.

Put now $\mathcal{G}:=\operatorname{SL}(2, \mathbb{C}) \ltimes \mathbb{R}^{4}$ which acts on $\widehat{\mathbb{R}}^{4}$ by means of the dual action canonically given by the semi-direct product structure of $\widetilde{\mathcal{P}_{+}^{\uparrow}}$. It is defined by

$$
\widetilde{\gamma}: \operatorname{SL}(2, \mathbb{C}) \ltimes \mathbb{R}^{4} \rightarrow \text { Aut } \widehat{\mathbb{R}}^{4} \text { and }\left(\widetilde{\gamma}_{(A, a)} \chi\right)(x):=\chi\left(\Lambda_{A}^{-1}(x)\right),
$$

where $\chi \in \widehat{\mathbb{R}}^{4}, A \in \mathrm{SL}(2, \mathbb{C}), a, x \in \mathbb{R}^{4}$. For a fixed character $\chi \in \widehat{\mathbb{R}}^{4}$ consider $M$ as the orbit generated by the previous action and the corresponding isotropy subgroup is given by

$$
\mathcal{G}_{\chi}:=\left\{(A, a) \in \mathrm{SL}(2, \mathbb{C}) \ltimes \mathbb{R}^{4} \mid \widetilde{\gamma}_{(A, a)} \chi=\chi\right\} \quad \text { and recall that } \widetilde{\mathcal{P}_{+}^{\uparrow}} / \mathcal{G}_{\chi} \cong \mathbb{D} .
$$

We have now the principal $\mathcal{G}_{\chi}$-bundle and the associated bundle given respectively by

$$
\mathcal{B}_{1}:=\left(\widetilde{\mathcal{P}_{+}^{\uparrow}}, \operatorname{pr}_{1}, \mathbb{D}\right) \text { and } \mathcal{B}_{2}\left(\tau^{(\mathrm{can})}\right):=\left(\widetilde{\mathcal{P}_{+}^{\uparrow}} \times_{\mathcal{G}_{\chi}} \mathcal{H}, \mathrm{pr}_{2}, \mathbb{D}\right),
$$

where $\tau^{(\mathrm{can})}$ is a unitary representation of $\mathcal{G}_{\chi}$ on $\mathcal{H}$. If $\tau^{(\mathrm{can})}$ is irreducible, then the corresponding induced representation, which is called the canonical representation, is irreducible. Even more, every irreducible representation of $\mathcal{G}$ is obtained (modulo unitary equivalence) in this way. Recall also that the canonical representation is unitary iff $\tau^{\text {(can) }}$ is unitary. 
2.3 Remark In the present context relativistic wave equations appear if one considers reducible representations of the isotropy group $\mathcal{G}_{\chi}$. On the subspace of solutions of these equations (which can be consequently characterised by projections in $\mathcal{H}$ ) the reducible induced representation will turn irreducible. We will study relativistic wave equations in the following for massive and massless representations. We will show that these have a fundamentally different character as a consequence of the complementary properties of the respective little groups. (Here we use the name little group to denote the subgroup of $\mathrm{SL}(2, \mathbb{C})$ appearing in the isotropy group.) Indeed, the representations of the massive little group considered will be unitary and fully decomposable, while the corresponding representations of the massless little group will not satisfy these properties. Massive relativistic wave equations will be characterised by reducing projections, while the massless ones are associated to invariant (but not reducing) projections.

\subsection{Massive canonical representations}

Choose a character $\chi_{\breve{p}}$, with $\breve{p}:=(m, 0,0,0), m>0$, i.e. $\chi_{\breve{p}}(a)=e^{-i \breve{p} a}$, where $a \in \mathbb{R}^{4}$ and $\breve{p} a$ means the Minkowski scalar product. In this case we have $\mathcal{G}_{\chi_{\breve{p}}}=\mathrm{SU}(2) \ltimes \mathbb{R}^{4}$. As unitary representation of the isotropy subgroup on $\mathcal{H}^{\left(\frac{n}{2}, 0\right)}$ we take

$$
\tau^{(\mathrm{can})}(U, a):=e^{-i \breve{p} a} D^{\left(\frac{n}{2}, 0\right)}(U), \quad(U, a) \in \mathcal{G}_{\chi_{\breve{p}}} .
$$

We can now consider (omitting for simplicity the index $\left(\frac{n}{2}, 0\right)$ ) the bundles,

$$
\mathcal{B}_{1}^{(\mathrm{can})}:=\left(\widetilde{\mathcal{P}_{+}^{\uparrow}}, \operatorname{pr}_{1}, \mathfrak{C}_{m}^{+}\right) \quad \text { and } \quad \mathcal{B}_{2}\left(\tau^{(\mathrm{can})}\right):=\left(\widetilde{\mathcal{P}_{+}^{\uparrow}} \times_{\mathcal{G}_{\chi_{\breve{p}}}} \mathcal{H}, \operatorname{pr}_{2}, \mathfrak{C}_{m}^{+}\right)
$$

where we have used the diffeomorphism $\widetilde{\mathcal{P}_{+}^{\uparrow}} / \mathcal{G}_{\chi_{\breve{p}}} \cong \mathfrak{C}_{m}^{+}:=\left\{p \in \mathbb{R}^{4} \mid p^{2}=m^{2}, p^{0}>0\right\}$ between the factor space and the positive mass shell $\mathfrak{C}_{m}^{+} . \mu(\mathrm{d} p)$ denotes the corresponding invariant measure on $\mathfrak{C}_{m}^{+}$.

The principal $\mathcal{G}_{\chi_{\breve{p}}}$-bundle $\mathcal{B}_{1}^{(\mathrm{can})}$ is trivial [18]. Indeed, a global continuous section $s$ is

$$
s: \mathfrak{C}_{m}^{+} \longrightarrow \widetilde{\mathcal{P}_{+}^{\uparrow}}, \quad s(p):=\left(H_{p}, 0\right) \in \mathrm{SL}(2, \mathbb{C}) \ltimes \mathbb{R}^{4}=\widetilde{\mathcal{P}_{+}^{\uparrow}},
$$

where $H_{p}:=\frac{1}{\sqrt{2 m\left(m+p_{0}\right)}}(m+P), P=\sum_{\mu} p_{\mu} \sigma_{\mu}$ [12, Section A.1] and $\sigma_{\mu}, \mu=0,1,2,3$, are the unit and the Pauli matrices. The assignment $p \rightarrow P$ defines a vector space isomorphism between $\mathbb{R}^{4}$ and the set of self-adjoint elements in $\operatorname{Mat}_{2}(\mathbb{C})$.

So, once the section $s$ is fixed and using $s(p)^{-1}(A, a) s(q)=\left(H_{p}^{-1} A H_{q}, \Lambda_{H_{p}^{-1}} a\right) \in \mathcal{G}_{\breve{p}}$, as well as $q=\Lambda_{A}^{-1} p \in \mathfrak{C}_{m}^{+}$, we have on $\mathrm{L}^{2}\left(\mathfrak{C}_{m}^{+}, \mathcal{H}, \mu(\mathrm{d} p)\right)$ the massive canonical representation (cf. Eq. (므),

$$
\left(V^{\text {(can) }}(g) \varphi\right)(p)=e^{-i p a} D\left(H_{p}^{-1} A H_{q}\right) \varphi(q),
$$

where $g=(A, a) \in \mathrm{SL}(2, \mathbb{C}) \ltimes \mathbb{R}^{4}$ and $\varphi \in \mathrm{L}^{2}\left(\mathfrak{C}_{m}^{+}, \mathcal{H}, \mu(\mathrm{d} p)\right)$. The element $H_{p}^{-1} A H_{q} \in \mathrm{SU}(2)$ is called the Wigner rotation (e.g. [74, Section 2.3]). $V^{(\mathrm{can})}$ is unitary w.r.t. usual $\mathrm{L}^{2}$-scalar product,

$$
\left\langle\varphi_{1}, \varphi_{2}\right\rangle:=\int_{\mathfrak{C}_{m}^{+}}\left\langle\varphi_{1}(p), \varphi_{2}(p)\right\rangle_{\mathcal{H}} \mu(\mathrm{d} p) .
$$

2.4 Remark The representation $\left(\mathrm{L}^{2}\left(\mathfrak{C}_{m}^{+}, \mathcal{H}, \mu(\mathrm{d} p)\right), V^{\text {(can) }},\langle\cdot, \cdot\rangle\right)$ is equivalent to the representation $\left(h_{m}, V_{1},\langle\cdot, \cdot\rangle_{\beta}\right)$ used in [12, Section A.1], where we define first for $\varphi, \psi$ a pair of $\mathcal{H}$-valued functions the sesquilinear form

$$
\langle\varphi, \psi\rangle_{\beta}:=\int_{\mathfrak{C}_{m}^{+}}\langle\varphi(p), \beta(p) \psi(p)\rangle_{\mathcal{H}} \mu(\mathrm{d} p),
$$


with

$$
\begin{aligned}
\beta(p) & :=D^{\left(\frac{n}{2}, 0\right)}\left(P^{\dagger}\right)=\stackrel{n}{\otimes} P^{\dagger} \\
P^{\dagger} & :=\frac{1}{m}\left(p_{0} \sigma_{0}-\sum_{i=1}^{3} p_{i} \sigma_{i}\right)=\left(H_{p}^{-1}\right)^{*} H_{p}^{-1}
\end{aligned}
$$

Then we put

$$
h_{m}:=\left\{\varphi: \mathfrak{C}_{m}^{+} \longrightarrow \mathcal{H} \mid \varphi \text { is Borel and }\langle\varphi, \varphi\rangle_{\beta}<\infty\right\} .
$$

Finally, the representation

$$
\left(V_{1}(g) \varphi\right)(p):=e^{-i p a} D^{\left(\frac{n}{2}, 0\right)}(A) \varphi(q)
$$

for $g=(A, a) \in \widetilde{\mathcal{P}_{+}^{\uparrow}}=\operatorname{SL}(2, \mathbb{C}) \ltimes \mathbb{R}^{4}, \varphi \in h_{m}$ and $q:=\Lambda_{A}^{-1} p$, is unitary w.r.t. $\langle\cdot, \cdot\rangle_{\beta}$. Roughly, we have removed the $H_{p}$-matrices from the definition of $V_{1}$ at the price of introducing a positive operator-valued function on $\mathfrak{C}_{m}^{+}, p \mapsto \beta(p)$, in the definition of the corresponding scalar product. This equivalent definition of canonical representation has been very useful in order to construct massive free nets [12. We will also adapt this idea to the massless case.

The equivalence of the representations mentioned above is given explicitly by the following isometry $\Psi: \mathrm{L}^{2}\left(\mathfrak{C}_{m}^{+}, \mathcal{H}, \mu(\mathrm{d} p)\right) \rightarrow h_{m}$,

$$
(\Psi \varphi)(p):=D\left(H_{p}\right) \varphi(p)
$$

and it is immediately checked that $\Psi V^{(\text {can })}(g)=V_{1}(g) \Psi, g \in \widetilde{\mathcal{P}_{+}^{\uparrow}}$.

2.5 Remark It is now easy to relate the algebraically reducible representation $T^{(\text {cov) }}$ in (7) with the canonical representation $V^{(\mathrm{can})}$ given in Eq. (11). Indeed, recalling the definitions introduced in Remark 2.4, we consider the embedding

$$
\mathfrak{I}: \mathcal{S}\left(\mathbb{R}^{4}, \mathcal{H}\right) \longrightarrow h_{m}
$$

defined as Fourier transformation, $\hat{f}(p)=\int_{\mathbb{R}^{4}} e^{-i p x} f(x) d^{4} x$, and restriction to $\mathfrak{C}_{m}^{+}$(recall 58 , Section IX.9]). Then the equation

$$
\mathfrak{I} T^{\text {(cov) }}(g)=V_{1}(g) \mathfrak{I}, \quad g \in \widetilde{\mathcal{P}_{+}^{\uparrow}},
$$

holds. From the preceding Remark we already know that $V_{1}$ and $V^{\text {(can) }}$ are equivalent.

\subsection{Massive relativistic wave equations}

As already mentioned in Remark 2.3] relativistic wave equations appear when one considers reducible representations of the little group $\mathrm{SU}(2)$. They serve to reduce the corresponding induced representation and therefore it is natural to associate with relativistic wave equations corresponding projections on $\mathcal{H}$. We will also illustrate the preceding results with two examples that have nontrivial spin, namely the Dirac and the Proca Equations.

Niederer and O'Rafeartaigh present Eq. (20.8) in [54 as "... the most general covariant wave equation corresponding to a given (nonzero) mass and spin ..." (see Definition 2.7 below). It is useful to recognise that in [54] and [10. Chapter 21] the spaces $\left(h_{m},\langle\cdot, \cdot\rangle_{\beta}\right)$ of Remark 2.4 are essentially used. In our context we can equivalently write the mentioned equation also for the space $\left(\mathrm{L}^{2}, V^{(\mathrm{can})}\right)$. Using Eq. (16) the following result is a straightforward consequence of the mentioned equivalence. 
2.6 Lemma Let $\pi$ be a reducing orthoprojection w.r.t. $\tau^{(\mathrm{can})}$, i.e. $\pi \tau^{(\mathrm{can})}(g)=\tau^{(\mathrm{can})}(g) \pi, g \in$ $\mathrm{SU}(2) \ltimes \mathbb{R}^{4}$. Then the following equations are equivalent:

(i) $\pi \widetilde{\varphi}(p)=\widetilde{\varphi}(p)$, for $\widetilde{\varphi} \in \mathrm{L}^{2}\left(\mathfrak{C}_{m}^{+}, \mathcal{H}, \mu(\mathrm{d} p)\right)$.

(ii) $\pi(p) \varphi(p)=\varphi(p)$, where $\pi(p):=D\left(H_{p}\right) \pi D\left(H_{p}\right)^{-1}$ and $\varphi(p):=D\left(H_{p}\right) \widetilde{\varphi}(p) \in h_{m}, p \in \mathfrak{C}_{m}^{+}$.

Following Niederer and O'Rafeartaigh we introduce the notion of massive relativistic wave equation (see [54, $\S 20$ and $\S 21]$ for further details and motiviation).

2.7 Definition Let $\pi$ be a reducing orthoprojection w.r.t. $\tau^{(\mathrm{can})} \uparrow\left(\mathrm{SU}(2) \ltimes \mathbb{R}^{4}\right)$. Then we call

$$
\pi(p) \varphi(p)=\varphi(p), \quad \varphi \in h_{m}, p \in \mathfrak{C}_{m}^{+},
$$

a massive relativistic wave equation associated with $\pi$ (cf. Lemma [2.6 (ii)).

2.8 Remark - The equation in the previous definition coincides with [54, Eq. (20.8)] or [10, Eq. (17) of Section 21.1]. Specifying $\tau^{(\mathrm{can})}$ and $\pi$ in this context one obtains the conventional massive relativistic wave equations written in momentum space. For convenience of the reader we mention the examples of Dirac's and Proca's equation. More examples of massive relativistic wave equations and the corresponding projections are summarised in [54, Table 2].

- Note further, that the subspace of $h_{m}$ characterised by the equation in Lemma 2.6 (ii) is $V_{1}$-invariant. Use for example the relation:

$$
D(A)^{-1} \pi(p) D(A)=\pi\left(\Lambda_{A}^{-1} p\right) .
$$

Thus we have seen that the reducing subspaces of $\mathcal{H}$ are in correspondence with relativistic wave equations. Recall that by compactness the unitary representation $D^{\left(\frac{j}{2}, \frac{k}{2}\right)}\lceil\mathrm{SU}(2)$, $(j, k) \neq(0,0)$, is fully decomposable, i.e. it can be decomposed as a direct sum of irreducible subrepresentations.

The Dirac Equation [64, Section 9]: Take $\mathcal{H}:=\mathbb{C}^{4}$ and $\tau(A):=\left(\begin{array}{cc}A & 0 \\ 0 & \left(A^{*}\right)^{-1}\end{array}\right) \in \operatorname{SL}(4, \mathbb{C})$ for $A \in \mathrm{SL}(2, \mathbb{C})$. As reducible inducing representation we use $\tau(U, a):=e^{-i \breve{p} a} \tau(U), \quad(U, a) \in$ $\mathrm{SU}(2) \ltimes \mathbb{R}^{4}$. Now, consider the orthoprojection

$$
\pi^{\text {(Dirac) }}:=\frac{1}{2}\left(\begin{array}{llll}
1 & 0 & 1 & 0 \\
0 & 1 & 0 & 1 \\
1 & 0 & 1 & 0 \\
0 & 1 & 0 & 1
\end{array}\right),
$$

which satisfies $\pi^{\text {(Dirac) }} \tau(U)=\tau(U) \pi^{\text {(Dirac) }}, U \in \mathrm{SU}(2)$, and using the isometry $\Psi$ as well as the notation $\varphi(p):=(\Psi \widetilde{\varphi})(p)=\tau\left(H_{p}\right) \widetilde{\varphi}(p)$ we can reformulate the preceding lemma in the present context as:

2.9 Proposition With the preceding notation we have that $\varphi$ satisfies the Dirac Equation iff $\widetilde{\varphi}$ satisfies $\pi^{\text {(Dirac) }} \widetilde{\varphi}(p)=\widetilde{\varphi}(p), p \in \mathfrak{C}_{m}^{+}$.

Proof: First of all we note that from Lemma 2.6. $\pi^{(\text {Dirac })} \widetilde{\varphi}(p)=\widetilde{\varphi}(p)$ iff $\pi^{(\text {Dirac })}(p) \varphi(p)=\varphi(p)$, where $\pi^{\text {(Dirac) }}(p):=\tau\left(H_{p}\right) \pi^{\text {(Dirac) }} \tau\left(H_{p}\right)^{-1}$. We calculate

$$
\pi^{\text {(Dirac) }}(p)=\frac{1}{2 m}\left(\begin{array}{cc}
m \mathbb{1} & P \\
\widehat{P} & m \mathbb{1}
\end{array}\right),
$$


where $P:=p_{0} \sigma_{0}+\sum_{i=1}^{3} p_{i} \sigma_{i}$ and $\widehat{P}:=p_{0} \sigma_{0}-\sum_{i=1}^{3} p_{i} \sigma_{i}$, so that $P \widehat{P}=p^{2} \mathbb{1}=m^{2} \mathbb{1}$. Denoting $\gamma(p):=$ $\left(\begin{array}{ll}0 & P \\ \widehat{P} & 0\end{array}\right)$ we obtain $\pi^{(\text {Dirac })}(p)=\frac{1}{2 m}(m \mathbb{1}+\gamma(p))$ and thus $\pi^{(\text {Dirac })}(p) \varphi(p)=\varphi(p)$ iff $\gamma(p) \varphi(p)=$ $m \varphi(p)$, which is the Dirac equation in momentum space notation.

The Proca Equation [54]: In the present case we put $\mathcal{H}:=\mathbb{C}^{2} \otimes \mathbb{C}^{2} \cong \mathbb{C}^{4}$ and as inducing representation we use

$$
\tau(U, a):=e^{-i \breve{p} a} D^{\left(\frac{1}{2}, \frac{1}{2}\right)}(U)=e^{-i \breve{p} a} U \otimes \bar{U}, \quad(U, a) \in \mathrm{SU}(2) \ltimes \mathbb{R}^{4} .
$$

As an orthoprojection we take,

$$
\pi^{\text {(Proca) }}:=\frac{1}{2}\left(\begin{array}{rccc}
1 & 0 & 0 & -1 \\
0 & 2 & 0 & 0 \\
0 & 0 & 2 & 0 \\
-1 & 0 & 0 & 1
\end{array}\right),
$$

which satisfies $\pi^{\text {(Proca) }} \tau(U)=\tau(U) \pi^{\text {(Proca) }}, U \in \mathrm{SU}(2)$. Further details, e.g. the explicit relation between the canonical and covariant descriptions in this particular case can be found in [17.

\subsection{Massless canonical representations}

To specify massless representations with discrete helicity we choose a character $\chi_{\breve{p}}, \breve{p}:=$ $(1,0,0,1) \in \mathcal{C}_{+}\left(\right.$the mantle of the forward light cone), i.e. $\chi_{\breve{p}}(a)=e^{-i \breve{p} a}, a \in \mathbb{R}^{4}$ and $\breve{p} a$ means the Minkowski scalar product. A straightforward computation shows that the isotropy subgroup is given by $\mathcal{G}_{\chi_{\breve{p}}}=\mathcal{E}(2) \ltimes \mathbb{R}^{4}$, where the two-fold cover of the 2-dimensional Euclidean group is

$$
\mathcal{E}(2):=\left\{\left(\begin{array}{cc}
e^{\frac{i}{2} \theta} & e^{-\frac{i}{2} \theta} z \\
0 & e^{-\frac{i}{2} \theta}
\end{array}\right) \in \operatorname{SL}(2, \mathbb{C}) \mid \theta \in[0,4 \pi), z \in \mathbb{C}\right\} .
$$

The little group $\mathcal{E}(2)$ is noncompact and since its commutator subgroup is already abelian it follows that $\mathcal{E}(2)$ is solvable. Further, it has again the structure of a semi-direct product. (In contrast with these facts we have that the massive little group $\mathrm{SU}(2)$ is compact and simple.) Since $\mathcal{E}(2)$ is a connected and solvable Lie group we know from Lie's Theorem (cf. [10]) that the only finite-dimensional irreducible representations are 1-dimensional, i.e. $\mathcal{H}:=\mathbb{C}$. Therefore in order to induce irreducible and unitary representations of the whole group that describe discrete helicity values we define

$$
\tau^{(\mathrm{can})}(L, a):=e^{-i \bar{p} a}\left(e^{\frac{i}{2} \theta}\right)^{n},
$$

where $(L, a) \in \mathcal{E}(2) \ltimes \mathbb{R}^{4}=\mathcal{G}_{\chi_{\breve{p}}}, n \in \mathbb{N}$. Note that this representation is not faithful. Indeed, the normal subgroup $\left\{\left(\begin{array}{ll}1 & z \\ 0 & 1\end{array}\right) \mid z \in \mathbb{C}\right\}$ is trivially represented (see also [72, Section II]). Some authors associate this subgroup to certain gauge degrees of freedom of the system (e.g. 36, 41, 63]). We consider next the bundles,

$$
\mathcal{B}_{1}^{(\text {can })}:=\left(\widetilde{\mathcal{P}_{+}^{\uparrow}}, \operatorname{pr}_{1}, \mathcal{C}_{+}\right) \text {and } \mathcal{B}_{2}\left(\tau^{(\text {can })}\right):=\left(\widetilde{\mathcal{P}_{+}^{\uparrow}} \times_{\mathcal{G}_{\breve{p}}} \mathbb{C}, \operatorname{pr}_{2}, \mathcal{C}_{+}\right)
$$

where we have used the diffeomorphism $\widetilde{\mathcal{P}_{+}^{\uparrow}} / \mathcal{G}_{\chi_{\breve{p}}} \cong \mathcal{C}_{+}:=\left\{p \in \mathbb{R}^{4} \mid p^{2}=0, p^{0}>0\right\}$ between the factor space and the mantle of the forward light cone. We denote by $\mu_{0}(\mathrm{~d} p)$ the corresponding invariant measure on $\mathcal{C}_{+}$. 
In contrast with the massive case the bundle $\mathcal{B}_{1}^{\text {(can) }}$ has no global continuous section. The reason for this lies in the following topological obstruction: if $\mathcal{B}_{1}^{(\mathrm{can})}$ had a global continuous section (hence would be a trivial bundle), then the $n$-th homotopy groups $\Pi_{n}(\cdot), n \in \mathbb{N}$, of the total space would be equal to the direct sum of the homotopy groups of the isotropy subgroup and of the base manifold. We can check in particular for the second homotopy group that on the one hand

$$
\Pi_{2}(\mathcal{G})=\Pi_{2}\left(S^{3}\right)=0
$$

and on the other hand

$$
\Pi_{2}\left(\mathcal{G}_{\chi_{\breve{p}}}\right) \oplus \Pi_{2}\left(\mathcal{C}_{+}\right)=\Pi_{2}\left(S^{1}\right) \oplus \Pi_{2}\left(S^{2}\right)=\mathbb{Z}
$$

(see [18] for further details).

Nevertheless, we can specify a Borel section considering a continuous one in a chart that does not include the set $\left\{p \in \mathcal{C}_{+} \mid p_{3}=-p_{0}\right\}$ (which is of measure zero w.r.t. $\mu_{0}(\mathrm{~d} p)$ ). Putting $\mathcal{C}_{+}^{\circ}:=\mathcal{C}_{+} \backslash\left\{p \in \mathcal{C}_{+} \mid p_{3}=-p_{0}\right\}$ a (local) continuous section is given explicitly by

$$
s: \mathcal{C}_{+}^{\circ} \longrightarrow \widetilde{\mathcal{P}_{+}^{\uparrow}}, \quad s(p):=\left(H_{p}, 0\right) \in \mathrm{SL}(2, \mathbb{C}) \ltimes \mathbb{R}^{4}=\widetilde{\mathcal{P}_{+}^{\uparrow}},
$$

where

$$
H_{p}:=\frac{1}{\sqrt{2 p_{0}\left(p_{0}+p_{3}\right)}}\left(\begin{array}{cc}
-\sqrt{p_{0}}\left(p_{0}+p_{3}\right) & \frac{p_{1}-i p_{2}}{\sqrt{p_{0}}} \\
-\sqrt{p_{0}}\left(p_{1}+i p_{2}\right) & -\frac{p_{0}+p_{3}}{\sqrt{p_{0}}}
\end{array}\right) .
$$

Recall that the $H_{p}$-matrices satisfy the equation

$$
H_{p}\left(\begin{array}{ll}
2 & 0 \\
0 & 0
\end{array}\right) H_{p}^{*}=P, \quad \text { where } \quad P=\left(\begin{array}{cc}
p_{0}+p_{3} & p_{1}-i p_{2} \\
p_{1}+i p_{2} & p_{0}-p_{3}
\end{array}\right)=p_{0} \sigma_{0}+\sum_{i=1}^{3} p_{i} \sigma_{i} .
$$

We use here, as in the massive case, the vector space isomorphism between $\mathbb{R}^{4}$ and the set of self-adjoint elements in $\operatorname{Mat}_{2}(\mathbb{C})$.

2.10 Remark The representation spaces of the canonical representations are typically $L^{2}$-spaces and therefore it is enough to consider fixed Borel sections as above. The fact that we are allowed to choose a continuous section of the corresponding bundle $\mathcal{B}_{1}$ in the massive case is an other pleasant and characteristic feature of these models.

From the point of view of quantum fields (to be defined in Section 4) what is crucial is the fact that the singularity of (20) does not affect the continuity (w.r.t. to the Schwartz and $L^{2}$-topologies) of the embedding that intertwines the covariant and the massless canonical representations. Indeed, in Theorem 4.2 we give a detailed proof of the mentioned continuity for the embedding associated to the Weyl case and that uses explicitly the section defined in Eq. (20). This ensures that the 2-point distributions defined by the corresponding massless quantum fields are tempered.

If we consider the section in Eq. (20) fixed, then we have on $\mathrm{L}^{2}\left(\mathcal{C}_{+}, \mathbb{C}, \mu_{0}(\mathrm{~d} p)\right)$ the canonical massless representations (cf. Eq. (5))

$$
\left(U_{ \pm}(g) \varphi\right)(p)=e^{-i p a}\left(e^{ \pm \frac{i}{2} \theta(A, p)}\right)^{n} \varphi(q)
$$

where $g=(A, a) \in \mathrm{SL}(2, \mathbb{C}) \ltimes \mathbb{R}^{4}, n \in \mathbb{N}, q:=\Lambda_{A}^{-1} p$ and for $A=\left(\begin{array}{ll}a & b \\ c & d\end{array}\right) \in \operatorname{SL}(2, \mathbb{C})$ we compute

$$
e^{-\frac{i}{2} \theta(A, p)}:=\left(H_{p}^{-1} A H_{q}\right)_{22}=\frac{-b\left(p_{1}+i p_{2}\right)+d\left(p_{0}+p_{3}\right)}{\left|-b\left(p_{1}+i p_{2}\right)+d\left(p_{0}+p_{3}\right)\right|} .
$$


$U_{ \pm}$are unitary w.r.t. usual $\mathrm{L}^{2}$-scalar product, satisfy the spectrum condition and the helicity of the model carrying one of these representations is $\pm \frac{n}{2}$.

2.11 Remark In contrast with the massive case, it is now clear that in order to relate the covariant representation with the canonical massless representation it will not be enough to consider the Fourier transformation of suitable test functions and its restriction to $\mathcal{C}_{+}$(cf. Remark 2.5). Indeed, the fibres of $\mathcal{B}_{2}\left(\tau^{(\mathrm{can})}\right)$ are 1-dimensional, while the fibres of $\mathcal{B}_{2}\left(\tau^{(\mathrm{cov})}\right)$ are at least 2-dimensional if one chooses a nontrivial inducing representation $\tau^{\text {(cov) }}$. In other words, if the models describe nontrivial helicity, then some further restriction must be performed on the fibres in order to reduce the covariant representation to the unitary and irreducible canonical one (for a more detailed analysis of this reduction see Subsection [2.6). There are at least three ways to perform the mentioned reduction:

(i) One possibility of restricting the dimension of the fibres is to work on the space of solutions of the massless relativistic wave equations (cf. Remark 2.3). We will follow this alternative in the following subsections and present some explicit examples.

(ii) A second possibility is to use certain semidefinite sesquilinear forms and the reduction is done by means of the factor spaces that can be naturally constructed from the degeneracy subspaces of the sesquilinear form. This possibility is studied in [12, Part B] and [48] (cf. also Remark 2.13).

(iii) Finally one can perform the mentioned reduction for the bosonic models at the $\mathrm{C}^{*}$-level by the so-called $T$-procedure of Grundling and Hurst [30] (see [46, 47] for details). Imposing 'quantum constraints' on the $\mathrm{C}^{*}$-algebra level will show to be equivalent to consider the space of solutions of massless relativistic wave equations as reference space of the corresponding CCR-algebra.

In the next section the embedding $\mathfrak{I}$ intertwining the covariant and massless canonical representations (including the corresponding fibre reduction) will be the fundamental entity. In fact, $\mathfrak{I}$ specifies completely the corresponding net of $\mathrm{C}^{*}$-algebras satisfying Haag-Kastler's axioms. Each possibility to carry out the reduction described in (i)-(iii) above, requires its own embedding. Nontheless the corresponding nets of local $\mathrm{C}^{*}$-algebras turn out to be equivalent (cf. Remark 3.24 (i)).

\subsection{Massless relativistic wave equations}

In the present subsection we will extend Niederer and O'Rafeartaigh's systematic analysis of massive relativistic wave equations to the massless case (recall e.g. Definition 2.7). We will point out the fundamental differences between these two case.

We begin showing that, in contrast with the massive case (cf. Subsection 2.3), and due to the mathematical nature of the massless little group one has to give up the central notion of reducing projection for the inducing representation. In fact, we will show that, in the context of massless canonical representations, the useful objects are the invariant (but not reducing) projections of the corresponding inducing representations: Motivated by the form of the covariant representation in Eq. (7) (the one we want to reduce) we will consider as inducing representations of the isotropy subgroup

$$
\tau(L, a):=e^{-i \breve{p} a} D^{\left(\frac{j}{2}, \frac{k}{2}\right)}(L), \quad(L, a) \in \mathcal{E}(2) \ltimes \mathbb{R}^{4},
$$

which act on the Hilbert space $\mathcal{H}^{\left(\frac{j}{2}, \frac{k}{2}\right)}$ of dimension $(j+1)(k+1)$. (In the rest of this subsection we will denote again $D^{\left(\frac{j}{2}, \frac{k}{2}\right)}(\cdot)$ simply by $D(\cdot)$ etc.) These representations are nonunitary 
and reducible ${ }^{1}$ for any $(j, k) \neq(0,0)$. They are also not fully decomposable [62, [10, p. 607] in contrast to the massive case, i.e. they can not be decomposed as direct sum of irreducible subrepresentations. Therefore, no nontrivial reducing projection $\pi$ will exist ${ }^{2}$ in this context (we will compute explicitly some intertwiner spaces in the following section).

Therefore by the general theory, the corresponding induced representation of $\widetilde{\mathcal{P}_{+}^{\uparrow}}$, which is given for $g=(A, a) \in \mathrm{SL}(2, \mathbb{C}) \ltimes \mathbb{R}^{4}, q:=\Lambda_{A}^{-1} p \in \mathcal{C}_{+}^{\circ}, \varphi \in \mathrm{L}^{2}\left(\mathcal{C}_{+}, \mathcal{H}, \mu_{0}(\mathrm{~d} p)\right)$ by

$$
(\widetilde{V}(g) \varphi)(p)=e^{-i p a} D\left(H_{p}^{-1} A H_{q}\right) \varphi(q), \quad H_{p}^{-1} A H_{q} \in \mathcal{E}(2),
$$

will also be nonunitary and reducible.

2.12 Remark Before studying in detail the following examples of massless relativistic wave equations we will introduce the massless analogue to the useful space $\left(h_{m},\langle\cdot, \cdot\rangle_{\beta}\right)$ presented in the context of massive representations in Remark 2.4 for $\varphi, \psi$ a pair of $\mathcal{H}$-valued functions the sesquilinear form,

$$
\langle\varphi, \psi\rangle_{+}:=\int_{\mathcal{C}_{+}}\left\langle\varphi(p), \widetilde{\beta_{+}}(p) \psi(p)\right\rangle_{\mathcal{H}} \mu_{0}(\mathrm{~d} p),
$$

where $\widetilde{\beta_{+}}(p):=D\left(H_{p}^{-1}\right)^{*} D\left(H_{p}^{-1}\right)$ and $H_{p}, p \in \mathcal{C}_{+}^{\circ}$, is defined in Eq. (21). Then we put

$$
h_{0}:=\left\{\varphi: \mathcal{C}_{+} \longrightarrow \mathcal{H} \mid \varphi \text { is Borel and }\langle\varphi, \varphi\rangle_{+}<\infty\right\} .
$$

Finally, the representation

$$
(V(g) \varphi)(p):=e^{-i p a} D(A) \varphi(q),
$$

for $g=(A, a) \in \widetilde{\mathcal{P}_{+}^{\uparrow}}=\operatorname{SL}(2, \mathbb{C}) \ltimes \mathbb{R}^{4}, \varphi \in h_{0}$ and $q:=\Lambda_{A}^{-1} p \in \mathcal{C}_{+}^{\circ}$, is equivalent to the representation $\widetilde{V}$ defined in (24), i.e. there exists an isometry, $\Psi: \mathrm{L}^{2}\left(\mathcal{C}_{+}, \mathcal{H}, \mu_{0}(\mathrm{~d} p)\right) \longrightarrow h_{0}$, given by

$$
(\Psi \varphi)(p):=D\left(H_{p}\right) \varphi(p)
$$

such that, $\Psi \widetilde{V}(g)=V(g) \Psi, g \in \widetilde{\mathcal{P}_{+}^{\uparrow}}$. The representation $V$ is also reducible and nonunitary w.r.t. $\langle\cdot, \cdot\rangle_{+}$.

2.13 Remark If we require the representation $V$ to leave the sesquilinear form $\langle\cdot, \cdot\rangle_{+}$invariant, then we are forced (cf. [9]) to redefine the operator-valued function $\widetilde{\beta_{+}}$above as

$$
\beta_{+}(p):=D\left(\overline{H_{p}^{-1}}\right)^{*} D\left(\left(\begin{array}{ll}
0 & 0 \\
0 & 1
\end{array}\right)\right) D\left(\overline{H_{p}^{-1}}\right)=: D\left(\overline{P^{\dagger}}\right), \quad \text { with } P^{\dagger}=\frac{1}{2}\left(p_{0} \sigma_{0}-\sum_{i=1}^{3} p_{i} \sigma_{i}\right),
$$

which is only a semidefinite operator on $\mathcal{H}$ for each $p \in \mathcal{C}_{+}^{\circ}$, (compare with the massive case in Remark [2.4). This option is taken in [48] (cf. also [12, Part B]), and has as a consequence that the necessary reduction that must be performed to compare the covariant with the canonical representation (cf. Remark 2.11) is done by means of the factor spaces that can be naturally constructed from the degeneracy subspace of the sesquilinear form $\langle\cdot, \cdot\rangle_{\beta_{+}}$. This redefinition of the $\beta$-functions is related with the fact of imposing massless relativistic wave equations, which is what we will examine below.

\footnotetext{
${ }^{1}$ Wigner already observes in [78 p. 670] using a different formal approach that the massless wave equations cannot be obtained in general from the massive ones by putting $m=0$. He also mentions that the irreducible and invariant linear manifold of states corresponding to the massive representations turns reducible for $m=0$ and considering the value of the spin bigger than $\frac{1}{2}$.

${ }^{2}$ This is possibly a reason why in [54 Sections 22-25] the authors do not follow the elegant approach used to describe the massive wave equations, when they study the massless case.
} 
Since in the present context no nontrivial reducing projections exist we will need to introduce the following family of invariant projections:

2.14 Definition From the set of all projections in $\mathcal{H}$, select those orthoprojections $\pi$ that are invariant w.r.t. $D\left\lceil\mathcal{E}(2)\right.$ and satisfy the equation ${ }^{3}$

$$
\pi D(L)^{*} D(L) \pi=\pi, \quad L \in \mathcal{E}(2) .
$$

2.15 Theorem Let $\pi$ be as in the preceding definition and put

$$
\widetilde{h}:=\left\{\varphi \in \mathrm{L}^{2}\left(\mathcal{C}_{+}, \mathcal{H}, \mu_{0}(\mathrm{~d} p)\right) \mid \pi \widetilde{\varphi}(p)=\widetilde{\varphi}(p)\right\} .
$$

Then $\widetilde{h}$ is a closed $\widetilde{V}$-invariant subspace of $\mathrm{L}^{2}\left(\mathcal{C}_{+}, \mathcal{H}, \mu_{0}(\mathrm{~d} p)\right)$ and $\widetilde{V}(g) \Gamma \widetilde{h}, g \in \widetilde{\mathcal{P}_{+}^{\uparrow}}$, is unitary. Further if $D(\mathcal{E}(2))$ is irreducible on $\pi \mathcal{H}$, then $\widetilde{V} \Gamma \widetilde{h}$ is also irreducible.

Proof: It is obvious that $\widetilde{h}$ is a closed subspace and since for $g=(A, a) \in \widetilde{\mathcal{P}_{+}^{\uparrow}}$ we have $H_{p}^{-1} A H_{q} \in$ $\mathcal{E}(2), q:=\Lambda_{A}^{-1} p$, the invariance follows for $\varphi \in \widetilde{h}$ from

$$
\begin{aligned}
\pi(\tilde{V}(g) \varphi)(p) & =e^{-i p a} \pi D\left(H_{p}^{-1} A H_{q}\right) \varphi(q)=e^{-i p a} \pi D\left(H_{p}^{-1} A H_{q}\right) \pi \varphi(q) \\
& =e^{-i p a} D\left(H_{p}^{-1} A H_{q}\right) \pi \varphi(q)=(\widetilde{V}(g) \varphi)(p) .
\end{aligned}
$$

Further, for $\varphi, \psi \in \widetilde{h}$ we also have

$$
\begin{aligned}
\langle\widetilde{V}(g) \varphi, \widetilde{V}(g) \psi\rangle & =\int_{\mathcal{C}_{+}}\left\langle D\left(H_{p}^{-1} A H_{q}\right) \varphi(q), D\left(H_{p}^{-1} A H_{q}\right) \psi(q)\right\rangle_{\mathcal{H}} \mu_{0}(\mathrm{~d} p) \\
& =\int_{\mathcal{C}_{+}}\left\langle\varphi(q), \pi D\left(H_{p}^{-1} A H_{q}\right)^{*} D\left(H_{p}^{-1} A H_{q}\right) \pi \psi(q)\right\rangle_{\mathcal{H}} \mu_{0}(\mathrm{~d} p) \\
& =\langle\varphi, \psi\rangle .
\end{aligned}
$$

Here we have used Eq. (28) and the invariance of $\mu_{0}$. The irreducibility statement follows from the general theory of induced representations stated before in this section.

The condition $\pi \widetilde{\varphi}(p)=\widetilde{\varphi}(p)$ used before can be rewritten in terms of the equivalent space $\left(h_{0}, V,\langle\cdot, \cdot\rangle_{+}\right)$of Remark 2.12. This will give the massless relativistic wave equations written in its usual form.

2.16 Lemma Let $\pi$ be an invariant, orthoprojection w.r.t. $\tau$, i.e. $\pi \tau(g) \pi=\tau(g) \pi$ for all $g \in$ $\mathcal{E}(2) \ltimes \mathbb{R}^{4}$. Then the following equations are equivalent:

(i) $\pi \widetilde{\varphi}(p)=\widetilde{\varphi}(p)$, for $\widetilde{\varphi} \in \mathrm{L}^{2}\left(\mathcal{C}_{+}, \mathcal{H}, \mu_{0}(\mathrm{~d} p)\right)$.

(ii) $\pi(p) \varphi(p)=\varphi(p)$, where $\pi(p):=D\left(H_{p}\right) \pi D\left(H_{p}\right)^{-1}$ and $\varphi(p):=D\left(H_{p}\right) \widetilde{\varphi}(p) \in h_{0}, p \in \mathcal{C}_{+}^{\circ}$.

2.17 Definition Let $\pi$ be an invariant orthoprojection w.r.t. $\tau^{(\mathrm{can})} \uparrow\left(\mathcal{E}(2) \ltimes \mathbb{R}^{4}\right)$. Then we call

$$
\pi(p) \varphi(p)=\varphi(p), \quad \varphi \in h_{0}, p \in \mathcal{C}_{+}^{\circ},
$$

a massless relativistic wave equation associated with $\pi$ (cf. Lemma [2.16 (ii)).

\footnotetext{
${ }^{3}$ Note that this condition is trivially satisfied by the reducing projections chosen in the massive case.
} 
The equation in (ii) is the generalisation to the massless case of Eq. (ii) in Lemma 2.6 (cf. also [54, Eq. (20.8)] or [10, Eq. (17) of Section 21.1]). In the following we will consider it in different particular cases and show in Corollary 2.25 that it includes the general form of massless relativistic wave equations.

2.18 Remark (a) As announced in the begining of this subsection the equation introduced in Definition 2.17 extends neatly to the massless case, the work of Niederer and O'Rafeartaigh concerning massive relativistic wave equation [54]. The mentioned equation contains as special cases the conventional massless relativistic wave equations written in momentum space. (See the following Weyl and Maxwell cases below, Corollary 2.25 and the table in Subsection 2.10)

The existence of the corresponding projections $\pi$ in the massive and massless cases is guaranteed by the fact that $\mathrm{SU}(2)$ resp. $\mathcal{E}(2)$ are compact resp. solvable Lie groups. Indeed, any finite-dimensional unitary representation $\tau^{(\text {can })} \uparrow\left(\mathrm{SU}(2) \ltimes \mathbb{R}^{4}\right)$ can be decomposed as a direct sum of irreducible ones (cf. [39, Theorem 27.30]). Moreover, since $\mathcal{E}(2)$ is solvable and connected, Lie's Theorem [10, p. 200] guarantees the existence of one-dimensional orthoprojections invariant w.r.t. $\tau^{\text {(can) }} \uparrow\left(\mathcal{E}(2) \ltimes \mathbb{R}^{4}\right)$.

(b) Notice that in certain steps in this subsection we have make use of nonunitary representations (see e.g. Eqs. (24) or (26)). Therefore these representations lie outside of Mackey's theory of induced representations. Nevertheless in these cases we do not use any result of this theory. The justification of this procedure (and the importance of the massless relativistic wave equations) comes from Theorem 2.15. In fact, here we turn back to the description of unitary and irreducible representations and these must be unitarily equivalent to the ones considered by Wigner. The equivalence is given explicitly in the Weyl and Maxwell cases in Propositions 2.21] and 2.24. (For the existence of projections satisfying Eq. (28) recall the previous item.)

(c) Note finally the fundamentally different role that massless wave equations play (in contrast to the massive ones) when reducing the covariant representation. Indeed, massive relativistic wave equations appear when we consider reducible representations of $\mathrm{SU}(2)$ and therefore will not be present if we choose e.g. the irreducible representations given by $D^{\left(\frac{n}{2}, 0\right)}\left\lceil\mathrm{SU}(2)\right.$. On the contrary $D^{\left(\frac{j}{2}, \frac{k}{2}\right)} \uparrow \mathcal{E}(2)$ is always reducible if nontrivial helicity is admitted and therefore the space of solutions of massless relativistic wave equations is unavoidable if we want to work in momentum space with irreducible canonical representations. Indeed, in Sections 2.7 and 2.8, the Weyl- and Maxwell's equations will naturally appear when considering $D^{\left(\frac{n}{2}, 0\right)}\lceil\mathcal{E}(2), n=1,2$.

\subsection{Conditions on the intertwining operator and fibre reduction}

It is useful at this point to complete Remark 2.11] on the fibre reduction and study in detail the intertwing operator $\mathfrak{I}$ between the covariant and massless canonical representation with nontrivial discrete helicity. By Wigner's analysis and, in particular, due to the dual action defined in Eq. (8), it is clear that $\mathfrak{I}$ must contain the Fourier transformation - This is also the case in massive models (see Remark 2.5. for scalar models see also [58, Section IX.9]). We can now decompose the intertwining operator in its constituents. (The case with helicity $\frac{1}{2}$ (hence $\mathcal{H}=\mathbb{C}^{2}$ ) is already typical.)

$$
\mathcal{S}\left(\mathbb{R}^{4}, \mathbb{C}^{2}\right) \stackrel{\widehat{\longrightarrow}}{\longrightarrow}\left(\widehat{\mathbb{R}^{4}}, \mathbb{C}^{2}\right) \stackrel{R}{\longrightarrow} C^{\infty}\left(\left(\mathbb{R}^{3} \backslash 0\right), \mathbb{C}^{2}\right) \stackrel{M}{\longrightarrow} C^{\infty}\left(\left(\mathbb{R}^{3} \backslash 0\right), \mathbb{C}\right) \subset L^{2}\left(\left(\mathbb{R}^{3} \backslash 0\right), \mathbb{C}\right),
$$

where $R$ is the resctriction operator onto $\mathcal{C}_{+},(R \widehat{f})(\mathbf{p}):=\widehat{f}(|\mathbf{p}|, \mathbf{p}), \mathbf{p} \in\left(\mathbb{R}^{3} \backslash 0\right)$, and $M$ is the operator performing the fibre reduction mentioned above. The latter operator is characteristic 
for massless models with nontrial helicity. (In order to keep argument transparent we work here with the massless representation $U$ considered in Eq. (23).) The conditions on the intertwining operator $\mathfrak{I}$, which is the composition of the preceding chain of mappings, must satisfy the following conditions:

(a) $\mathfrak{I}$ must be linear (hence $M$ must be linear).

(b) I must be continuous between the Schwartz and the Hilbert space topologies (recall Remark 2.10).

(c) I must intertwine the covariant and massless canonical representations, i.e.

$$
\mathfrak{I} T(g)=U(g) \mathfrak{I}, \quad g \in \widetilde{\mathcal{P}_{+}^{\uparrow}} .
$$

Some concrete examples of intertwining operators satisfying the above conditions in the Weyl and Maxwell cases are specified in Eqs. (60) and (62).

\subsection{The Weyl Equation}

We begin with the simplest representation of $\mathcal{E}(2)$ with dimension bigger than one (see e.g. 37, Section V.A]). For $L=\left(\begin{array}{cc}e^{\frac{i}{2} \theta} & e^{-\frac{i}{2} \theta} z \\ 0 & e^{-\frac{i}{2} \theta}\end{array}\right) \in \mathcal{E}(2)$ we have on $\mathcal{H}^{\left(\frac{1}{2}, 0\right)}:=\mathbb{C}^{2}$ the representation given by $D^{\left(\frac{1}{2}, 0\right)}(L):=L$. (Notation: In the remaining subsection we will denote the representation $D^{\left(\frac{1}{2}, 0\right)}(\cdot)$ simply by $D(\cdot)$. We will also skip the index $\left(\frac{1}{2}, 0\right)$ from the objects associated to $D(\cdot)$, e.g. the representation $V$, the scalar product $\langle\cdot, \cdot\rangle$ etc., in order to keep the notation simple.) The only nontrivial $D(\mathcal{E}(2))$-invariant subspace is $\mathbb{C}\left(\begin{array}{l}1 \\ 0\end{array}\right)$ and we choose the corresponding invariant orthoprojection $\pi:=\left(\begin{array}{ll}1 & 0 \\ 0 & 0\end{array}\right)$. In the following lemma we will establish the relation between the equation in Lemma 2.16 (ii) and the Weyl Equation.

2.19 Lemma Put $\varphi(p):=H_{p} \widetilde{\varphi}(p) \in h_{0}$, for all $\widetilde{\varphi} \in \mathrm{L}^{2}\left(\mathcal{C}_{+}, \mathbb{C}^{2}, \mu_{0}(\mathrm{~d} p)\right)$ (see Eq. (27)). Then we have that $\widetilde{\varphi}$ satisfies the equation $\pi \widetilde{\varphi}(p)=\widetilde{\varphi}(p)$ iff $\varphi$ satisfies the Weyl Equation.

Proof: 1 . Suppose that $\widetilde{\varphi} \in \mathrm{L}^{2}\left(\mathcal{C}_{+}, \mathbb{C}^{2}, \mu_{0}(\mathrm{~d} p)\right)$ satisfies $\pi \widetilde{\varphi}(p)=\widetilde{\varphi}(p), p \in \mathcal{C}_{+}$. Then there exists a scalar function $\chi \in \mathrm{L}^{2}\left(\mathcal{C}_{+}, \mathbb{C}, \mu_{0}(\mathrm{~d} p)\right)$ such that $\widetilde{\varphi}(p)=\left(\begin{array}{c}\chi(p) \\ 0\end{array}\right)$. Next we write the Weyl operator in momentum space as

$$
\mathcal{W}(p):=\left(p_{0} \sigma_{0}-\sum_{i=1}^{3} p_{i} \sigma_{i}\right)
$$

and notice that we can rewrite $\mathcal{W}(p), p \in \mathcal{C}_{+}^{\circ}$, as

$$
\mathcal{W}(p)=\left(H_{p}^{-1}\right)^{*}\left(\begin{array}{ll}
0 & 0 \\
0 & 2
\end{array}\right) H_{p}^{-1}=2 P^{\dagger} .
$$

Therefore, $\varphi(p)=H_{p}\left(\begin{array}{c}\chi(p) \\ 0\end{array}\right)$, satisfies the Weyl Equation $\mathcal{W}(p) \varphi(p)=\mathcal{W}(p) H_{p}\left(\begin{array}{c}\chi(p) \\ 0\end{array}\right)=0$. Recall also that in terms of the spinorial components the Weyl equation is usually written as

$$
\sum_{C=0}^{1}\left(P^{\dagger}\right)_{C^{\prime} C} \varphi^{C}(p)=\sum_{C=0}^{1}\left(P^{\dagger}\right)_{C^{\prime} C}\left(H_{p}\right)_{0}^{C} \widetilde{\varphi}^{0}(p)=0, \quad C^{\prime} \in\{0,1\} .
$$


2. Suppose on the other hand that $\varphi(p)=H_{p} \widetilde{\varphi}(p) \in h_{0}$, with $\widetilde{\varphi}(p)=\left(\begin{array}{l}\widetilde{\varphi}_{1}(p) \\ \widetilde{\varphi}_{2}(p)\end{array}\right)$, satisfies the Weyl Equation $\mathcal{W}(p) \varphi(p)=0$. Then $\widetilde{\varphi}_{2}=0$ and $\widetilde{\varphi}$ satisfies the equation $\pi \widetilde{\varphi}(p)=\widetilde{\varphi}(p)$.

The space of solutions of the Weyl equation is therefore given by

$$
h_{+}:=\left\{H_{p}\left(\begin{array}{c}
\chi(p) \\
0
\end{array}\right) \mid \chi \in \mathrm{L}^{2}\left(\mathcal{C}_{+}, \mathbb{C}, \mu_{0}(\mathrm{~d} p)\right)\right\},
$$

2.20 Lemma Define on the space of solutions of the Weyl Equation $h_{+}$the scalar product

$$
\left\langle\varphi_{1}, \varphi_{2}\right\rangle_{+}:=\int_{\mathcal{C}_{+}}\left\langle\varphi_{1}(p),\left(H_{p}^{-1}\right)^{*} H_{p}^{-1} \varphi_{2}(p)\right\rangle_{\mathbb{C}^{2}} \mu_{0}(\mathrm{~d} p), \quad \text { for } \quad \varphi_{i} \in h_{+}, i=1,2 .
$$

The representation given for $g=(A, a) \in \mathrm{SL}(2, \mathbb{C}) \ltimes \mathbb{R}^{4}, q:=\Lambda_{A}^{-1} p$ and $\varphi \in h_{+}$by

$$
\left(V_{1}(g) \varphi\right)(p):=e^{-i p a} A \varphi(q)
$$

is unitary w.r.t. $\langle\cdot, \cdot\rangle_{+}$and irreducible.

Proof: First note that for $\varphi_{i}(p)=H_{p}\left(\begin{array}{c}\chi_{i}(p) \\ 0\end{array}\right), \chi_{i} \in \mathrm{L}^{2}\left(\mathcal{C}_{+}, \mathbb{C}, \mu_{0}(\mathrm{~d} p)\right), i=1,2$,

$$
\left\langle\varphi_{1}, \varphi_{2}\right\rangle_{+}=\int_{\mathcal{C}_{+}} \overline{\chi_{1}(p)} \chi_{2}(p) \mu_{0}(\mathrm{~d} p)
$$

Since $\pi \mathcal{H}$ is 1-dimensional and $\pi L^{*} L \pi=\pi, L \in \mathcal{E}(2)$, Theorem 2.15 completes the proof.

Next we establish the equivalence between the representation $\left(h_{+}, V_{1},\langle\cdot, \cdot\rangle_{+}\right)$defined above and the representation $\left(\mathrm{L}^{2}\left(\mathcal{C}_{+}, \mathbb{C}, \mu_{0}(\mathrm{~d} p)\right), U_{+},\langle\cdot, \cdot\rangle_{\mathrm{L}^{2}}\right)$ : The canonical representation for $n=1$ (cf. Eq. (23) $)$ is

$$
\left(U_{+}(g) \chi\right)(p):=e^{-i p a} e^{\frac{i}{2} \theta(A, p)} \chi(q), \quad \chi \in \mathrm{L}^{2}\left(\mathcal{C}_{+}, \mathbb{C}, \mu_{0}(\mathrm{~d} p)\right)
$$

for $q:=\Lambda_{A}^{-1} p$ and $g=(A, a) \in \mathrm{SL}(2, \mathbb{C}) \ltimes \mathbb{R}^{4} . U_{+}(\cdot)$ is irreducible, strongly continuous and unitary for the usual $\mathrm{L}^{2}$-scalar product, $\langle\cdot, \cdot\rangle_{\mathrm{L}^{2}}$. With the preceding notation we have the following equivalence of representations

2.21 Proposition The mapping $\Phi_{+}: h_{+} \longrightarrow \mathrm{L}^{2}\left(\mathcal{C}_{+}, \mathbb{C}, \mu_{0}(\mathrm{~d} p)\right)$ defined by,

$$
\Phi_{+}\left(H_{(\cdot)}\left(\begin{array}{c}
\chi(\cdot) \\
0
\end{array}\right)\right)(p)=\chi(p)
$$

is an isometric isomorphism between $\left(h_{+},\langle\cdot, \cdot\rangle_{+}\right)$and $\left(\mathrm{L}^{2}\left(\mathcal{C}_{+}, \mathbb{C}, \mu_{0}(\mathrm{~d} p)\right),\langle\cdot, \cdot\rangle_{\mathrm{L}^{2}}\right)$, that commutes with the corresponding representations, i.e. $\Phi_{+} V_{1}(g)=U_{+}(g) \Phi_{+}, g \in \widehat{\mathcal{P}_{+}^{\uparrow}}$.

Proof: That the mapping $\Phi_{+}$is an isometry follows already from Eq. (32) in the proof of the preceding lemma. The intertwining property is proved by direct computation. Indeed, for $g=$ $(A, a) \in \mathrm{SL}(2, \mathbb{C}) \ltimes \mathbb{R}^{4}$ and putting $q:=\Lambda_{A}^{-1} p$, we have on the one hand 


$$
\left(U_{+}(A, a) \Phi_{+}(\varphi)\right)(p)=e^{-i p a} e^{\frac{i}{2} \theta(A, p)} \chi(q)
$$

and on the other hand

$$
\begin{aligned}
\Phi_{+}\left(V_{1}(A, a) \varphi\right)(p) & =\Phi_{+}\left(e^{-i(\cdot) a} A H_{\Lambda_{A}^{-1}(\cdot)}\left(\begin{array}{c}
\chi\left(\Lambda_{A}^{-1}(\cdot)\right) \\
0
\end{array}\right)\right)(p) \\
& =\Phi_{+}\left(H_{(\cdot)} e^{-i(\cdot) a} H_{(\cdot)}^{-1} A H_{\Lambda_{A}^{-1}(\cdot)}\left(\begin{array}{c}
\chi\left(\Lambda_{A}^{-1}(\cdot)\right) \\
0
\end{array}\right)\right)(p) \\
& =e^{-i p a} e^{\frac{i}{2} \theta(A, p)} \chi(q),
\end{aligned}
$$

where for the last equation we have used that $\left(H_{p}^{-1} A H_{q}\right)_{11}=e^{\frac{i}{2} \theta(A, p)}$.

With the preceding result we have also proved the equivalence between the representation $\left(h_{+}, V_{1},\langle\cdot, \cdot\rangle_{+}\right)$and the representation $\left(\mathfrak{H}_{-}^{\prime}, V_{4}^{\prime},\langle\cdot, \cdot\rangle_{\beta_{-}}^{\prime}\right)$ used in [12, Theorem B.2.17].

Let us finish this subsection defining the space and the representation corresponding to the opposite helicity. They will be denoted by the subindex "-" and the proofs are analogous as before. This representation space associated to the opposite helicity will be necessary in order to construct the reference space of the CAR-algebra (cf. Subsection 3.1).

On the space

$$
h_{-}:=\left\{\overline{H_{p}}\left(\begin{array}{c}
\chi(p) \\
0
\end{array}\right) \mid \chi \in \mathrm{L}^{2}\left(\mathcal{C}_{+}, \mathbb{C}, \mu_{0}(\mathrm{~d} p)\right)\right\}
$$

define for $g=(A, a) \in \mathrm{SL}(2, \mathbb{C}) \ltimes \mathbb{R}^{4}, q:=\Lambda_{A}^{-1} p$ and $\varphi \in h_{-}$the representation

$$
\left(V_{4}(g) \varphi\right)(p):=e^{-i p a} \bar{A} \varphi(q) .
$$

This representation is irreducible and unitary w.r.t. the scalar product,

$$
\left\langle\varphi_{1}, \varphi_{2}\right\rangle_{-}:=\int_{\mathcal{C}_{+}}\left\langle\varphi_{1}(p),\left({\overline{H_{p}}}^{-1}\right)^{*}{\overline{H_{p}}}^{-1} \varphi_{2}(p)\right\rangle_{\mathbb{C}^{2}} \mu_{0}(\mathrm{~d} p), \quad \text { for } \quad \varphi_{i} \in h_{-}, i=1,2 .
$$

Finally $\left(h_{-}, V_{4},\langle\cdot, \cdot\rangle_{-}\right)$is equivalent to $\left(\mathrm{L}^{2}\left(\mathcal{C}_{+}, \mathbb{C}, \mu_{0}(\mathrm{~d} p)\right), U_{-},\langle\cdot, \cdot\rangle_{\mathrm{L}^{2}}\right)$, where the latter representation is given by

$$
\left(U_{-}(g) \chi\right)(p):=e^{-i p a} e^{-\frac{i}{2} \theta(A, p)} \chi(q), \quad \chi \in \mathrm{L}^{2}\left(\mathcal{C}_{+}, \mathbb{C}, \mu_{0}(\mathrm{~d} p)\right), q:=\Lambda_{A}^{-1} p .
$$

\subsection{Maxwell Equations: $\mathfrak{F}$-Equation}

Now for any $L=\left(\begin{array}{cc}e^{\frac{i}{2} \theta} & e^{-\frac{i}{2} \theta} z \\ 0 & e^{-\frac{i}{2} \theta}\end{array}\right) \in \mathcal{E}(2)$ we have on $\mathcal{H}^{(1,0)}:=\operatorname{Sym}\left(\mathbb{C}^{2} \otimes \mathbb{C}^{2}\right)$ the representation

$$
D^{(1,0)}(L):=L \otimes L
$$

(Notation: In the remaining subsection we will denote again the representation $D^{(1,0)}(\cdot)$ simply by $D(\cdot)$ etc.). We select the 1-dimensional $D(\mathcal{E}(2)$ )-invariant subspace characterised by the orthoprojection $\pi:=\left(\begin{array}{ll}1 & 0 \\ 0 & 0\end{array}\right) \otimes\left(\begin{array}{ll}1 & 0 \\ 0 & 0\end{array}\right)$. 
2.22 Lemma Put $\varphi(p):=D\left(H_{p}\right) \widetilde{\varphi}(p) \in h_{0}, \widetilde{\varphi} \in \mathrm{L}^{2}\left(\mathcal{C}_{+}, \mathcal{H}, \mu_{0}(\mathrm{~d} p)\right)$ (see Eq. (27)). Then we have that $\widetilde{\varphi}$ satisfies the equation $\pi \widetilde{\varphi}(p)=\widetilde{\varphi}(p)$ iff $\varphi$ satisfies the spinorial form of Maxwell Equation (F-Equation for short), which in components is given by

$$
\sum_{C=0}^{1}\left(P^{\dagger}\right)_{C^{\prime} C} \varphi^{C B}(p)=0, \quad C^{\prime} \in\{0,1\}, B \in\{0,1\} .
$$

Proof: 1 . Note first that $\pi \widetilde{\varphi}(p)=\widetilde{\varphi}(p)$ iff $\widetilde{\varphi}^{01}(p)=\widetilde{\varphi}^{10}(p)=0=\widetilde{\varphi}^{11}(p)$. Therefore from Eq. (30) we get

$$
\sum_{C=0}^{1}\left(P^{\dagger}\right)_{C^{\prime} C} \varphi^{C B}(p)=\sum_{C=0}^{1}\left(P^{\dagger}\right)_{C^{\prime} C}\left(H_{p}\right)_{0}^{C}\left(H_{p}\right)_{0}^{B} \widetilde{\varphi}^{00}(p)=0, \quad C^{\prime} \in\{0,1\}, B \in\{0,1\} .
$$

2. Conversely, suppose that $\sum_{C=0}^{1}\left(P^{\dagger}\right)_{C^{\prime} C} \varphi^{C B}(p)=0$. From the form of $P^{\dagger}$ (see Eq. (29)) and since

$$
\widetilde{\varphi}^{E B}(p)=\left(H_{p}^{-1}\right)_{C}^{E}\left(H_{p}^{-1}\right)_{D}^{B} \varphi^{C D}(p)
$$

we obtain $\widetilde{\varphi}^{1 B}(p)=0, B \in\{0,1\}$, hence also $\widetilde{\varphi}^{01}(p)=0$. Therefore $\pi \widetilde{\varphi}(p)=\widetilde{\varphi}(p)$.

Recall that from a symmetric spinor field $\varphi^{C B}$ satisfying the $\mathfrak{F}$-Equation, one can construct canonically a real and antisymmetric tensor field $F_{\mu \nu}$ satisfying the source free Maxwell Equations [70. Exercise 13.3], [56, Section 5.1].

The space of solutions of the $\mathfrak{F}$-Equation is given by

$$
h_{+}:=\left\{D\left(H_{p}\right)\left(\begin{array}{l}
1 \\
0
\end{array}\right) \otimes\left(\begin{array}{l}
1 \\
0
\end{array}\right) \chi(p) \mid \chi \in \mathrm{L}^{2}\left(\mathcal{C}_{+}, \mathbb{C}, \mu_{0}(\mathrm{~d} p)\right)\right\},
$$

2.23 Lemma Define on the space of solutions of the $\mathfrak{F}$-Equation $h_{+}$the scalar product

$$
\left\langle\varphi_{1}, \varphi_{2}\right\rangle_{+}:=\int_{\mathcal{C}_{+}}\left\langle\varphi_{1}(p), D\left(H_{p}^{-1}\right)^{*} D\left(H_{p}^{-1}\right) \varphi_{2}(p)\right\rangle_{\mathbb{C}^{4}} \mu_{0}(\mathrm{~d} p), \quad \text { for } \quad \varphi_{i} \in h_{+}, i=1,2 .
$$

The representation given for $g=(A, a) \in \mathrm{SL}(2, \mathbb{C}) \ltimes \mathbb{R}^{4}, q:=\Lambda_{A}^{-1} p$ and $\varphi \in h_{+}$, by

$$
\left(V_{+}(g) \varphi\right)(p):=e^{-i p a} D(A) \varphi(q),
$$

is unitary w.r.t. $\langle\cdot, \cdot\rangle_{+}$and irreducible.

Proof: First note that for $\varphi_{i}(p)=D\left(H_{p}\right)\left(\begin{array}{l}1 \\ 0\end{array}\right) \otimes\left(\begin{array}{l}1 \\ 0\end{array}\right) \chi_{i}(p), \chi_{i} \in \mathrm{L}^{2}\left(\mathcal{C}_{+}, \mathbb{C}, \mu_{0}(\mathrm{~d} p)\right), i=1,2$,

$$
\left\langle\varphi_{1}, \varphi_{2}\right\rangle_{+}=\int_{\mathcal{C}_{+}} \overline{\chi_{1}(p)} \chi_{2}(p) \mu_{0}(\mathrm{~d} p) .
$$

Since the space $\pi \mathcal{H}$ is 1-dimensional and $\pi D(L)^{*} D(L) \pi=\pi, L \in \mathcal{E}(2)$, Theorem 2.15 completes the proof.

Next we establish the equivalence between $\left(h_{+}, V_{+},\langle\cdot, \cdot\rangle_{+}\right)$defined above and the representation $\left(\mathrm{L}^{2}\left(\mathcal{C}_{+}, \mathbb{C}, \mu_{0}(\mathrm{~d} p)\right), U_{+},\langle\cdot, \cdot\rangle_{\mathrm{L}^{2}}\right)$. Consider on the space $\mathrm{L}^{2}\left(\mathcal{C}_{+}, \mathbb{C}, \mu_{0}(\mathrm{~d} p)\right)$ the canonical representation for $n=2$ (cf. Eq. (23)),

$$
\left(U_{+}(g) \chi\right)(p):=e^{-i p a} e^{i \theta(A, p)} \chi(q), \quad \chi \in \mathrm{L}^{2}\left(\mathcal{C}_{+}, \mathbb{C}, \mu_{0}(\mathrm{~d} p)\right)
$$

for $q:=\Lambda_{A}^{-1} p$ and $g=(A, a) \in \mathrm{SL}(2, \mathbb{C}) \ltimes \mathbb{R}^{4} . U_{+}(\cdot)$ is irreducible, strongly continuous and unitary for the usual $\mathrm{L}^{2}$-scalar product $\langle\cdot, \cdot\rangle_{\mathrm{L}^{2}}$. With the preceding notation we have the following equivalence of representations 
2.24 Proposition The mapping $\Phi_{+}: h_{+} \longrightarrow \mathrm{L}^{2}\left(\mathcal{C}_{+}, \mathbb{C}, \mu_{0}(\mathrm{~d} p)\right)$ defined by,

$$
\Phi_{+}\left(D\left(H_{(\cdot)}\right)\left(\begin{array}{l}
1 \\
0
\end{array}\right) \otimes\left(\begin{array}{l}
1 \\
0
\end{array}\right) \chi(\cdot)\right)(p)=\chi(p), \quad p \in \mathcal{C}_{+}^{\circ},
$$

is an isometric isomorphism between $\left(h_{+},\langle\cdot, \cdot\rangle_{+}\right)$and $\left(\mathrm{L}^{2}\left(\mathcal{C}_{+}, \mathbb{C}, \mu_{0}(\mathrm{~d} p)\right),\langle\cdot, \cdot\rangle_{\mathrm{L}^{2}}\right)$ that commutes with the corresponding representations, i.e. $\Phi_{+} V_{+}(g)=U_{+}(g) \Phi_{+}, g \in \widetilde{\mathcal{P}_{+}^{\uparrow}}$.

Proof: That the mapping $\Phi_{+}$is an isometry follows already from Eq. (37) in the proof of the preceding lemma. The intertwining property is proved by a direct computation. Indeed, for $g=(A, a) \in \mathrm{SL}(2, \mathbb{C}) \ltimes \mathbb{R}^{4}$ and putting $q:=\Lambda_{A}^{-1} p$, we have on the one hand,

$$
\left(U_{+}(A, a) \Phi_{+}(\varphi)\right)(p)=e^{-i p a} e^{i \theta(A, p)} \chi(q)
$$

and on the other hand computing similarly as in the proof of Proposition 2.21

$$
\begin{aligned}
\Phi_{+}\left(V_{+}(A, a) \varphi\right)(p) & =\Phi_{+}\left(D\left(H_{(\cdot)}\right) e^{-i(\cdot) a} D\left(H_{(\cdot)}^{-1} A H_{\Lambda_{A}^{-1}(\cdot)}\right)\left(\begin{array}{l}
1 \\
0
\end{array}\right) \otimes\left(\begin{array}{l}
1 \\
0
\end{array}\right) \chi\left(\Lambda_{A}^{-1}(\cdot)\right)\right)(p) \\
& =e^{-i p a} e^{i \theta(A, p)} \chi(q),
\end{aligned}
$$

where for the last equation we have used that $D\left(H_{p}^{-1} A H_{q}\right)_{11}=e^{i \theta(A, p)}$.

Let us finish this subsection defining the space and the representation corresponding to the opposite helicity. They will be denoted by the subindex "--" and the proofs are similar as before. This representation space associated to the opposite helicity will be necessary in order to construct the reference space of the CCR-algebra.

On the space

$$
h_{-}:=\left\{D\left(\overline{H_{p}}\right)\left(\begin{array}{l}
1 \\
0
\end{array}\right) \otimes\left(\begin{array}{l}
1 \\
0
\end{array}\right) \chi(p) \mid \chi \in \mathrm{L}^{2}\left(\mathcal{C}_{+}, \mathbb{C}, \mu_{0}(\mathrm{~d} p)\right)\right\}
$$

define for $g=(A, a) \in \mathrm{SL}(2, \mathbb{C}) \ltimes \mathbb{R}^{4}, q:=\Lambda_{A}^{-1} p$ and $\varphi \in h_{-}$the representation

$$
\left(V_{-}(g) \varphi\right)(p):=e^{-i p a} D(\bar{A}) \varphi(q) .
$$

This representation is irreducible and unitary w.r.t. the scalar product

$$
\left\langle\varphi_{1}, \varphi_{2}\right\rangle_{-}:=\int_{\mathcal{C}_{+}}\left\langle\varphi_{1}(p), D\left({\overline{H_{p}}}^{-1}\right)^{*} D\left({\overline{H_{p}}}^{-1}\right) \varphi_{2}(p)\right\rangle_{\mathbb{C}^{4}} \mu_{0}(\mathrm{~d} p), \quad \text { for } \quad \varphi_{i} \in h_{-}, i=1,2 .
$$

Finally $\left(h_{-}, V_{-},\langle\cdot, \cdot\rangle_{-}\right)$is equivalent to $\left(\mathrm{L}^{2}\left(\mathcal{C}_{+}, \mathbb{C}, \mu_{0}(\mathrm{~d} p)\right), U_{-},\langle\cdot, \cdot\rangle_{\mathrm{L}^{2}}\right)$, where the latter representation is given by

$$
\left(U_{-}(g) \chi\right)(p):=e^{-i p a} e^{-i \theta(A, p)} \chi(q), \quad \chi \in \mathrm{L}^{2}\left(\mathcal{C}_{+}, \mathbb{C}, \mu_{0}(\mathrm{~d} p)\right), q:=\Lambda_{A}^{-1} p .
$$

Generalisation to arbitrary helicity: We collect in this paragraph the obvious generalisation of the previous analysis of the Weyl resp. Maxwell equations (which correspond to helicities $\pm \frac{1}{2}$ resp. \pm 1$)$ to the systems carrying arbitrary discrete helicity.

We begin characterising general massless relativistic wave equations corresponding to helicity $\frac{n}{2}$ (cf. [56, p. 375]). The following result contains as special cases Lemmas 2.19] and 2.22. 
2.25 Corollary Consider the $D^{\left(\frac{n}{2}, 0\right)}(\mathcal{E}(2))$-invariant subspace characterised by the one-

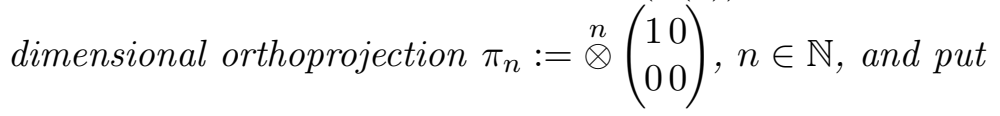

$$
\left.\varphi(p):=D^{\left(\frac{n}{2}, 0\right)}\left(H_{p}\right) \widetilde{\varphi}(p) \in h_{0}, \quad \widetilde{\varphi} \in \mathrm{L}^{2}\left(\mathcal{C}_{+}, \mathcal{H}, \mu_{0}(\mathrm{~d} p)\right) \quad \text { (cf. Eq. 27) }\right) .
$$

Then $\widetilde{\varphi}$ satisfies the equation $\pi \widetilde{\varphi}(p)=\widetilde{\varphi}(p)$ iff $\varphi$ satisfies the massless relativistic wave equation corresponding to helicity $\frac{n}{2}$. The latter equation is written in momentum space for the spinorial components as

$$
\sum_{C=0}^{1}\left(P^{\dagger}\right)_{C^{\prime} C} \psi^{C C_{1} \ldots C_{n-1}}(p)=0, \quad C_{1}, \ldots, C_{n-1} \in\{0,1\}, C^{\prime} \in\{0,1\} .
$$

2.26 Remark (i) The way of presenting relativistic wave equations written in momonetum space is justified by the group theoretical approach which is one of the basic ingredients of the present paper. However, to give a more complete picture of these equations we need to comment on them also as PDEs in position space, since they usually appear in the literature in this form. A general massless relativistic wave equations on position space is given by

$$
\sum_{C=0}^{1} \partial_{C^{\prime} C} \psi^{C C_{1} \ldots C_{n-1}}(x)=0, \quad C_{1}, \ldots, C_{n-1} \in\{0,1\}, C^{\prime} \in\{0,1\},
$$

where $\partial_{C^{\prime} C}$ is the first order differential operator on spinor fields corresponding to the usual gradient $\partial_{\mu}, \mu=0, \ldots, 3$ [70, Eq. 13.1.64]. It can be shown that Eq. (43) is equivalent to the usual wave equation $\square \psi^{C C_{1}} \ldots C_{n-1}(x)=0$, which is an hyperbolic equation, together with Eq. (43) holding only as an initial value constraint on a Cauchy surface (e.g. $x^{0}=0$ ) (for details see [70 pp. 376-377]). This fact confirms the point of view already stated in the introduction (see also Remark 2.11(i)) that massless relativistic wave equations can be seen as constraint equations restricting the fibre degrees of freedom.

The results cited above show that Eq. (43) has a well-posed initial value formulation is relevant if one wants to construct quantum fields on more general (globally hyperbolic) space-times, where the group theoretical approach is not possible due to the lack of symmetry.

(ii) We want now complete the generalisation to include the corrsponding spaces of solutions of the relativistic wave equations, the representations and the associated isometric isomorphisms. For this one needs only to replace the labels $(1,0)$ resp. $(0,1)$ by $\left(\frac{n}{2}, 0\right)$ resp. $\left(0, \frac{n}{2}\right)$, $n \geq 3$, in the present subsection. In particular we obtain in this way a characterisation of the Wigner massless Hilbert spaces with discrete helicity in terms of the space of solutions of the corresponding massless relativistic wave equation (cf. Corollary 2.25).

\subsection{Maxwell Equations: $\mathfrak{A}$-Equation}

For completeness we will include in our group theoretical context the discussion of Maxwell Equations in terms of the vector potential field (A-Equation). We will see that some techniques used in the previous subsection for treating Maxwell Equations in terms of the field strength (FEquation) will not be applicable anymore (cf. Remark 2.29). Instead we will use a Gupta-Bleuler like procedure will allow to establish an isometric isomorphism to the previous representation space. For a detailed treatment of quantum electromagnetism in terms of the vector potential (including constraints) we refer to [32]. 
For $L=\left(\begin{array}{cc}e^{\frac{i}{2} \theta} & e^{-\frac{i}{2} \theta} z \\ 0 & e^{-\frac{i}{2} \theta}\end{array}\right) \in \mathcal{E}(2)$ we have on $\mathcal{H}^{\left(\frac{1}{2}, \frac{1}{2}\right)}:=\mathbb{C}^{2} \otimes \mathbb{C}^{2} \cong \mathbb{C}^{4}$ the representation

$$
D^{\left(\frac{1}{2}, \frac{1}{2}\right)}(L):=L \otimes \bar{L} \cong\left(\begin{array}{cccc}
1 & e^{i \theta} \bar{z} & e^{-i \theta} z & |z|^{2} \\
0 & e^{i \theta} & 0 & z \\
0 & 0 & e^{-i \theta} & \bar{z} \\
0 & 0 & 0 & 1
\end{array}\right)
$$

(Notation: In the remaining subsection we will denote when no confusion arises again the representation $D^{\left(\frac{1}{2}, \frac{1}{2}\right)}(\cdot)$ simply by $\left.D(\cdot)\right)$. In order to include the nontrivial phases of the diagonal of $D(L)$ (recall that now we want to describe both helicity values \pm 1 ) one is forced to consider in this context the 3 -dimensional space characterised by the $D(\mathcal{E}(2))$-invariant orthoprojection $\pi:=\left(\begin{array}{cccc}1 & 0 & 0 & 0 \\ 0 & 1 & 0 & 0 \\ 0 & 0 & 1 & 0 \\ 0 & 0 & 0 & 0\end{array}\right)$

In the following lemma we will write the divergence equation $p_{0} \psi_{0}(p)-\sum_{i=1}^{3} p_{i} \psi_{i}(p)=0$ in an equivalent and for us more convenient form.

2.27 Lemma The vector $\varphi(p):=\left(\begin{array}{l}\varphi_{0}(p) \\ \varphi_{1}(p) \\ \varphi_{2}(p) \\ \varphi_{3}(p)\end{array}\right), p \in \mathcal{C}_{+}^{\circ}$, satisfies the equation

$$
-\left(p_{0}-p_{3}\right) \varphi_{0}(p)+\left(p_{1}+i p_{2}\right) \varphi_{1}(p)+\left(p_{1}-i p_{2}\right) \varphi_{2}(p)-\left(p_{0}+p_{3}\right) \varphi_{3}(p)=0
$$

iff the vector $\psi(p):=W_{s} \varphi(p)$, where $W_{s}:=\frac{1}{\sqrt{2}}\left(\begin{array}{rrrr}1 & 0 & 0 & 1 \\ 0 & 1 & 1 & 0 \\ 0 & i & -i & 0 \\ 1 & 0 & 0 & -1\end{array}\right)$ is a unitary matrix acting on $\mathbb{C}^{4}$, satisfies the equation $p_{0} \psi_{0}(p)-\sum_{i=1}^{3} p_{i} \psi_{i}(p)=0$.

Proof: The proof is straightforward since it uses essentially a unitary transformation acting on $\mathcal{H}$. We write it explicitly down in order to introduce some useful notation for later on. Put

$$
\eta:=W_{s}^{-1} \eta_{\text {Mink }} W_{s}=\left(\begin{array}{rrrc}
0 & 0 & 0 & -1 \\
0 & 1 & 0 & 0 \\
0 & 0 & 1 & 0 \\
-1 & 0 & 0 & 0
\end{array}\right), \quad \text { with } \quad \eta_{\text {Mink }}:=\left(\begin{array}{cccc}
1 & 0 & 0 & 0 \\
0 & -1 & 0 & 0 \\
0 & 0 & -1 & 0 \\
0 & 0 & 0 & -1
\end{array}\right) .
$$

Now for $p=\left(p_{0}, p_{1}, p_{2}, p_{3}\right) \in \mathcal{C}_{+}$and recalling that $\langle\cdot, \cdot\rangle_{\mathbb{C}^{4}}$ is antilinear in the first argument we have that the equations

$$
\left\langle p, \eta_{\mathrm{Mink}} \psi(p)\right\rangle_{\mathbb{C}^{4}}=\left\langle p, W_{s} \eta W_{s}^{-1} \psi(p)\right\rangle_{\mathbb{C}^{4}}=\left\langle W_{s}^{-1} p, \eta \varphi(p)\right\rangle_{\mathbb{C}^{4}}
$$

conclude the proof, since the last term is precisely $\frac{1}{\sqrt{2}}$ times the l.h.s. of Eq. (44).

In the following lemma we will establish the relation between the equation in Lemma 2.16 (ii) and the $\mathfrak{A}$-Equation (44).

2.28 Lemma Put $\varphi(p):=D\left(H_{p}\right) \widetilde{\varphi}(p) \in h_{0}$, for all $\widetilde{\varphi} \in \mathrm{L}^{2}\left(\mathcal{C}_{+}, \mathcal{H}, \mu_{0}(\mathrm{~d} p)\right.$ ) (see Eq. (27)). Then we have that $\widetilde{\varphi}$ satisfies the equation $\pi \widetilde{\varphi}(p)=\widetilde{\varphi}(p)$ iff $\varphi$ satisfies the $\mathfrak{A}$-Equation (44). 
Proof: 1 . Suppose that $\widetilde{\varphi}$ satisfies the equation $\pi \widetilde{\varphi}(p)=\widetilde{\varphi}(p), p \in \mathcal{C}_{+}^{\circ}$. Then there exist three scalar functions $\chi_{i} \in \mathrm{L}^{2}\left(\mathcal{C}_{+}, \mathbb{C}, \mu_{0}(\mathrm{~d} p)\right), i=0,1,2$, such that $\widetilde{\varphi}(p)=\left(\begin{array}{c}\chi_{0}(p) \\ \chi_{1}(p) \\ \chi_{2}(p) \\ 0\end{array}\right)$. But using

Eq. (21) one can explicitly check that $\varphi(p)=D\left(H_{p}\right) \widetilde{\varphi}(p)$ satisfies Eq. (44).

2. Suppose now that $\varphi$ satisfies Eq. (44). Then we can compute:

$$
\begin{aligned}
\widetilde{\varphi}_{3}(p) & =\left(D\left(H_{p}\right)^{-1} \varphi(p)\right)_{3} \\
& =\left(p_{0}-p_{3}\right) \varphi_{0}(p)-\left(p_{1}+i p_{2}\right) \varphi_{1}(p)-\left(p_{1}-i p_{2}\right) \varphi_{2}(p)+\left(p_{0}+p_{3}\right) \varphi_{3}(p) \\
& =0
\end{aligned}
$$

and therefore $\widetilde{\varphi}$ satisfies the equation $\pi \widetilde{\varphi}(p)=\widetilde{\varphi}(p), p \in \mathcal{C}_{+}^{\circ}$.

Denote by

$$
h_{+-}:=\left\{D\left(H_{p}\right)\left(\begin{array}{c}
\chi_{0}(p) \\
\chi_{1}(p) \\
\chi_{2}(p) \\
0
\end{array}\right) \mid \chi_{i} \in \mathrm{L}^{2}\left(\mathcal{C}_{+}, \mathbb{C}, \mu_{0}(\mathrm{~d} p)\right), i=0,1,2\right\}
$$

the space of solutions of the $\mathfrak{A}$-Equation and recall that from the general definition of $V$ given in Eq. (26) we have here,

$$
\left(V_{+-}(g) \varphi\right)(p):=e^{-i p a} D^{\left(\frac{1}{2}, \frac{1}{2}\right)}(A) \varphi(q),
$$

where $g=(A, a) \in \mathrm{SL}(2, \mathbb{C}) \ltimes \mathbb{R}^{4}, q:=\Lambda_{A}^{-1} p$ and $\varphi \in h_{+-}$.

2.29 Remark In analogy with the Weyl case or with the $\mathfrak{F}$-Equation case, we can try to define on $h_{+-}$the following sesquilinear form:

$$
\left\langle\varphi, \varphi^{\bullet}\right\rangle:=\int_{\mathcal{C}_{+}}\left\langle\varphi(p), D\left(H_{p}^{-1}\right)^{*} D\left(H_{p}^{-1}\right) \varphi^{\bullet}(p)\right\rangle_{\mathbb{C}^{4}} \mu_{0}(\mathrm{~d} p)=\int_{\mathcal{C}_{+}} \sum_{i=0}^{2} \overline{\chi_{i}(p)} \chi_{i}^{\bullet}(p) \mu_{0}(\mathrm{~d} p),
$$

where $\varphi(p):=D\left(H_{p}\right)\left(\begin{array}{c}\chi_{0}(p) \\ \chi_{1}(p) \\ \chi_{2}(p) \\ 0\end{array}\right), \varphi^{\bullet}(p):=D\left(H_{p}\right)\left(\begin{array}{c}\chi_{0}^{\bullet}(p) \\ \chi_{1}^{\bullet}(p) \\ \chi_{2}^{\bullet}(p) \\ 0\end{array}\right) \in h_{+-}$. This sesquilinear form is positive definite, but it will not be $V_{+_{-}}$-invariant, since in the present case we have in general that $\pi D\left(H_{p}^{-1} A H_{q}\right)^{*} D\left(H_{p}^{-1} A H_{q}\right) \pi \neq \pi$, cf. Eq. (28). Roughly speaking, we have allowed too many degrees of freedom on the fibre in order to apply the arguments used in the Weyl case or in the $\mathfrak{F}$-Equation case which are based on Theorem 2.15.

Motivated nevertheless by the Lorentz-invariance of the Minkowski scalar product we introduce the following sesquilinear form: for $\varphi, \varphi^{\bullet}$ as in the preceding lemma,

$$
\begin{aligned}
\left\langle\varphi, \varphi^{\bullet}\right\rangle_{+-} & :=\int_{\mathcal{C}_{+}}\left\langle\varphi(p), \eta \varphi^{\bullet}(p)\right\rangle_{\mathbb{C}^{4}} \mu_{0}(\mathrm{~d} p) \\
& =\int_{\mathcal{C}_{+}} \sum_{i=1}^{2} \overline{\chi_{i}(p)} \chi_{i}^{\bullet}(p) \mu_{0}(\mathrm{~d} p),
\end{aligned}
$$


where $\eta$ is given in the proof of Lemma 2.27 and can be seen as the spinorial form of the Minkowski metric. Note that since

$$
D(A)^{*} \eta D(A)=\eta
$$

the above sesquilinear form is $V_{+-}$-invariant, but now $\langle\cdot, \cdot\rangle_{+-}$is only semidefinite and the corresponding (degenerate) space of zero vectors is easily seen to be,

$$
h_{d}:=\left\{D\left(H_{p}\right)\left(\begin{array}{c}
\chi(p) \\
0 \\
0 \\
0
\end{array}\right) \mid \chi \in \mathrm{L}^{2}\left(\mathcal{C}_{+}, \mathbb{C}, \mu_{0}(\mathrm{~d} p)\right)\right\} .
$$

Since $h_{d}$ is a $V_{+-}$-invariant space denote by $V_{\mathfrak{A}}$ the natural definition of $V_{+-}$on the factor space $h_{\mathfrak{A}}:=h_{+-} / h_{d}$, which is Hilbert space w.r.t. the scalar product, $\langle\cdot, \cdot\rangle_{\mathfrak{A}}$, defined as the lift of $\langle\cdot, \cdot\rangle_{+-} \cdot{ }^{4}$ The elements of the factor space are written as $[\varphi]_{0}$, where $\varphi \in h_{+-}$and the bracket, $[\cdot]_{0}$, specify the corresponding equivalence class. The preceding situation with the appearance of factor spaces is typical when dealing with not fully decomposable representations of a Lie group. This situation is studied in general terms by Araki and our construction above using the space of solutions of the $\mathfrak{A}$-Equation is a special case of Theorem 1 in 4 .

The following statement justifies the use of the index +- in $h_{+_{-}}$, since this space carries a representation that contains the irreducible representations describing helicity +1 and -1 . In the next result we will show the equivalence of the representations $\left(V_{\mathfrak{A}}, h_{\mathfrak{A}}\right)$ and the direct sum of the canonical representations $U_{+}$and $U_{-}$given in Eqs. (39) and (42) respectively.

2.30 Proposition The mapping, $\Phi_{\mathfrak{A}}: h_{\mathfrak{A}} \longrightarrow \mathrm{L}^{2}\left(\mathcal{C}_{+}, \mathbb{C}, \mu_{0}(\mathrm{~d} p)\right) \oplus \mathrm{L}^{2}\left(\mathcal{C}_{+}, \mathbb{C}, \mu_{0}(\mathrm{~d} p)\right)$, defined by

$$
\left(\Phi_{\mathfrak{A}}\left(\left[D\left(H_{(\cdot)}\right)\left(\begin{array}{c}
\chi_{0}(\cdot) \\
\chi_{1}(\cdot) \\
\chi_{2}(\cdot) \\
0
\end{array}\right)\right]_{0}\right)\right)(p):=\chi_{1}(p) \oplus \chi_{2}(p),
$$

is an isometric isomorphism that commutes with the representations $V_{\mathfrak{A}}$ and $U_{+} \oplus U_{-}$, i.e. the equation, $\Phi_{\mathfrak{A}} V_{\mathfrak{A}}(g)=\left(U_{+}(g) \oplus U_{-}(g)\right) \Phi_{\mathfrak{A}}$ holds for all $g \in \widetilde{\mathcal{P}_{+}^{\uparrow}}$. The representation $V_{\mathfrak{A}}$ on $\left(h_{\mathfrak{A}},\langle\cdot, \cdot\rangle_{\mathfrak{A}}\right)$ is unitary, strongly continuous and reducible.

Proof: The unitarity of $V_{\mathfrak{A}}$ follows from the $V_{+-}$-invariance of the sesquilinear form $\langle\cdot, \cdot\rangle_{+-}$and from the construction of the factor space $h_{+-} / h_{d}$.

The isometry property of $\Phi_{\mathfrak{A}}$ follows already from Eq. (48) and the intertwining property can be checked by direct computation as in Proposition 2.24. Use, for instance, the relation

$$
\left(\Phi_{\mathfrak{A}}\left(\left[D\left(H_{(\cdot)}\right) e^{-i(\cdot) a} D\left(H_{(\cdot)}^{-1} A H_{\Lambda_{A}^{-1}(\cdot)}\right)\left(\begin{array}{c}
\chi_{0}\left(\Lambda_{A}^{-1} \cdot\right) \\
\chi_{1}\left(\Lambda_{A}^{-1} \cdot\right) \\
\chi_{2}\left(\Lambda_{A}^{-1} \cdot\right) \\
0
\end{array}\right)\right]_{0}\right)\right)(p)
$$

$$
=\left(U_{+}(g) \chi_{1}\right)(p) \oplus\left(U_{-}(g) \chi_{2}\right)(p) .
$$

${ }^{4}$ Notice that we can not restrict $V_{+-}$to the space $\left\{D\left(H_{p}\right)\left(\begin{array}{c}0 \\ \chi_{1}(p) \\ \chi_{2}(p) \\ 0\end{array}\right) \mid \chi_{i} \in \mathrm{L}^{2}\left(\mathcal{C}_{+}, \mathbb{C}, \mu_{0}(\mathrm{~d} p)\right), i=1,2\right\}$, because it is not $V_{+-}$-invariant. Indeed, this follows from the fact that the space $\left\{\left(\begin{array}{l}0 \\ a \\ b \\ 0\end{array}\right) \mid a, b \in \mathbb{C}\right\}$ is not $D(L)$ invariant, $L \in \mathcal{E}(2)$ (cf. [6. Section 5.B.1]). 
Recall further that, for $g=(A, a) \in \mathrm{SL}(2, \mathbb{C}) \ltimes \mathbb{R}^{4}, q:=\Lambda_{A}^{-1} p$ and $\chi \in \mathrm{L}^{2}\left(\mathcal{C}_{+}, \mathbb{C}, \mu_{0}(\mathrm{~d} p)\right)$, the canonical representations (cf. Eq. (23) in the case $n=2$ ),

$$
\left(U_{ \pm}(A, a) \chi\right)(p):=e^{-i p a} e^{ \pm i \theta(A, p)} \chi(q)
$$

are unitary, strongly continuous and irreducible. They correspond to systems with opposite helicity.

Finally, we will prove a theorem that relates the pair $\left(h_{\mathfrak{A}}, V_{\mathfrak{A}}\right)$ defined above with some combinations of the spaces and representations used in Subsection 2.8. Concretely, using the definitions $^{5}$ (35), (36), (40) and (41) we consider the following Hilbert space, scalar product and unitary representation w.r.t. it

$$
\left.\begin{array}{rl}
h_{\mathfrak{F}} & :=h_{+} \oplus h_{-} \\
\langle\cdot, \cdot\rangle_{\mathfrak{F}} & :=\langle\cdot, \cdot\rangle_{+} \oplus\langle\cdot, \cdot\rangle_{-} \\
V_{\mathfrak{F}} & :=V_{+} \oplus V_{-}
\end{array}\right\}
$$

Then we have the following equivalence between $\left(V_{\mathfrak{F}}, h_{\mathfrak{F}}\right)$ and $\left(V_{\mathfrak{A}}, h_{\mathfrak{A}}\right)$ :

2.31 Theorem The mapping $\Phi_{\mathfrak{A} \mathfrak{F}}: h_{\mathfrak{F}} \longrightarrow h_{\mathfrak{A}}$, defined by

$$
\Phi_{\mathfrak{A F}}\left(D^{(1,0)}\left(H_{(\cdot)}\right)\left(\begin{array}{l}
1 \\
0
\end{array}\right) \otimes\left(\begin{array}{l}
1 \\
0
\end{array}\right) \chi_{+}(p) \oplus D^{(0,1)}\left(H_{(\cdot)}\right)\left(\begin{array}{l}
1 \\
0
\end{array}\right) \otimes\left(\begin{array}{l}
1 \\
0
\end{array}\right) \chi_{-}(p)\right)(p):=\left[D^{\left(\frac{1}{2}, \frac{1}{2}\right)}\left(H_{p}\right)\left(\begin{array}{c}
0 \\
\chi_{+}(p) \\
\chi_{-}(p) \\
0
\end{array}\right)\right]_{0}
$$

with $\varphi_{+}(p):=D^{(1,0)}\left(H_{p}\right)\left(\begin{array}{l}1 \\ 0\end{array}\right) \otimes\left(\begin{array}{l}1 \\ 0\end{array}\right) \chi_{+}(p) \in h_{+}$and $\varphi_{-}(p):=D^{(0,1)}\left(H_{p}\right)\left(\begin{array}{l}1 \\ 0\end{array}\right) \otimes\left(\begin{array}{l}1 \\ 0\end{array}\right) \chi_{-}(p) \in h_{-}$, is an isometric isomorphism that commutes with the corresponding representations, i.e. $\Phi_{\mathfrak{A F}} V_{\mathfrak{F}}(g)=$ $V_{\mathfrak{A}}(g) \Phi_{\mathfrak{A} \mathfrak{F}}, g \in \widetilde{\mathcal{P}_{+}^{\uparrow}}$.

Proof: The isometry property follows from the equations,

$$
\begin{aligned}
\left\langle\Phi_{\mathfrak{A F}}\left(\varphi_{+} \oplus \varphi_{-}\right), \Phi_{\mathfrak{A F}}\left(\varphi_{+} \oplus \varphi_{-}\right)\right\rangle_{\mathfrak{A}} & =\int_{\mathcal{C}_{+}}\left(\left|\chi_{+}(p)\right|^{2}+\left|\chi_{-}(p)\right|^{2}\right) \mu_{0}(\mathrm{~d} p) \\
& =\left\langle\varphi_{+} \oplus \varphi_{-}, \varphi_{+} \oplus \varphi_{-}\right\rangle_{\mathfrak{F}}
\end{aligned}
$$

The intertwining property is a consequence of Proposition 2.24] (and the corresponding result for the opposite helicity) and of Proposition 2.30. Indeed, note that $\Phi_{\mathfrak{A F}}=\Phi_{\mathfrak{A}}^{-1} \Phi_{\mathfrak{F}}$ and, therefore,

$$
\Phi_{\mathfrak{A} \mathfrak{F}} V_{\mathfrak{F}}(g)=\Phi_{\mathfrak{A}}^{-1} \Phi_{\mathfrak{F}} V_{\mathfrak{F}}(g)=\Phi_{\mathfrak{A}}^{-1}\left(U_{+}(g) \oplus U_{-}(g)\right) \Phi_{\mathfrak{F}}=V_{\mathfrak{A}}(g) \Phi_{\mathfrak{A}}^{-1} \Phi_{\mathfrak{F}}=V_{\mathfrak{A}}(g) \Phi_{\mathfrak{A F}}, \quad g \in \widetilde{\mathcal{P}_{+}^{\uparrow}},
$$

and the proof is concluded.

2.32 Remark Landsman and Wiedemann [45, Theorem 1] interpret the space $\left(h_{\mathfrak{A}},\langle\cdot, \cdot\rangle_{\mathfrak{A}}, V_{\mathfrak{A}}\right)$ (written in tensorial language) in the context of Marsden-Weinstein reduction theory. They also mention its equivalence to the triplet $\left(h_{\mathfrak{F}},\langle\cdot, \cdot\rangle_{\mathfrak{F}}, V_{\mathfrak{F}}\right)$. For the relation between the two preceding spaces in terms of tensors see also [15] or [37, Section V.B].

\footnotetext{
${ }^{5}$ Recall that in Subsection $\left[2.8 D(\cdot)\right.$ means $D^{(1,0)}(\cdot)$. We have also written $D^{(0,1)}(A)$ instead of using the notation $D^{(1,0)}(\bar{A}), A \in \mathrm{SL}(2, \mathbb{C})$.
} 


\subsection{Summary}

In the present section we have described covariant and massive/massless canonical representations of the Poincaré group in the general frame of induced representation theory. Relativistic wave equations appear in the context of canonical representations if one considers reducible representations of the corresponding little groups. Due to the different nature of the massive resp. massless little groups, the corresponding relativistic wave equations play also a different role and are characterised by reducing resp. invariant projections. We will summerise in the following table some of the results concerning massive and massless relativistic wave equations.

\begin{tabular}{|c|c|c|}
\hline \multicolumn{3}{|c|}{ massive, $m>0$} \\
\hline REL. WAVE EQUATION & $\begin{array}{l}\text { INDUCING REP. OF } \mathrm{SU}(2) \text { : } \\
\text { Unitary and fully decomposable }\end{array}$ & REDUCING PROJECTION \\
\hline Dirac & $\tau(U):=U \oplus U$ & $\pi^{\text {(Dirac) }}:=\frac{1}{2}\left(\begin{array}{cccc}1 & 0 & 1 & 0 \\
0 & 1 & 0 & 1 \\
1 & 0 & 1 & 0 \\
0 & 1 & 0 & 1\end{array}\right)$ \\
\hline Proca & $D^{\left(\frac{1}{2}, \frac{1}{2}\right)}(U)=U \otimes \bar{U}$ & $\pi^{\text {(Proca) }}:=\frac{1}{2}\left(\begin{array}{cccc}1 & 0 & 0 & -1 \\
0 & 2 & 0 & 0 \\
0 & 0 & 2 & 0 \\
-1 & 0 & 0 & 1\end{array}\right)$ \\
\hline \multicolumn{3}{|c|}{ massless, $m=0$} \\
\hline REL. WAVE EQUATION & $\begin{array}{l}\text { INDUCING REP. OF } \mathcal{E}(2) \text { : } \\
\text { Nonunitary and non } \\
\text { fully decomposable }\end{array}$ & INVARIANT PROJECTION \\
\hline Weyl & $D^{\left(\frac{1}{2}, 0\right)}(L):=L$ & $\pi_{\mathfrak{W}}:=\left(\begin{array}{ll}1 & 0 \\
0 & 0\end{array}\right)$ \\
\hline Maxwell: $\mathfrak{F}$-Eq. & $D^{(1,0)}(L):=L \otimes L$ & $\pi_{\mathfrak{F}}:=\left(\begin{array}{ll}1 & 0 \\
0 & 0\end{array}\right) \otimes\left(\begin{array}{ll}1 & 0 \\
0 & 0\end{array}\right)$ \\
\hline Maxwell: $\mathfrak{A}$-Eq. & $D^{\left(\frac{1}{2}, \frac{1}{2}\right)}(L)=L \otimes \bar{L}$ & $\pi_{\mathfrak{A}}:=\left(\begin{array}{cccc}1 & 0 & 0 & 0 \\
0 & 1 & 0 & 0 \\
0 & 0 & 1 & 0 \\
0 & 0 & 0 & 0\end{array}\right)$ \\
\hline $\begin{array}{l}\text { General massless } \\
\text { (helicity }: \frac{n}{2}, n \geq 1 \text { ) }\end{array}$ & $D^{\left(\frac{n}{2}, 0\right)}(L)=\stackrel{n}{\otimes} L$ & $\pi_{n}:=\stackrel{n}{\otimes}\left(\begin{array}{ll}1 & 0 \\
0 & 0\end{array}\right)$ \\
\hline
\end{tabular}




\section{Massless free nets and relativistic wave equations}

We begin this section stating some core axioms of local quantum physics [34, 35, 11, 14, 159, 28. Here the point of view is that the correspondence $\mathcal{O} \mapsto \mathcal{A}(\mathcal{O})$ between Minkowski space regions $\mathcal{O}$ and local algebras of observables $\mathcal{A}(\mathcal{O})$ characterises intrinsically the theory. Following Haag's suggestive idea, quantum fields (which are the central objects in other formulations of QFT) can be seen in the present setting just as 'coordinates' of the preceding net, in the sense that one may use different quantum fields to describe the same abstract net. In order to avoid any concrete representation of the $\mathrm{C}^{*}$-algebra we will construct the net directly following the strategy presented in [12] (see also 60]), i.e. basing the construction on group-theoretical arguments and standard CAR or CCR-theory (see for the latter algebras [14, Chapter 8] and references cited therein; in the Fermi case we will use Araki's self-dual approach to the CAR-algebra). We will call the result of this construction a free net and the fundamental object that characterises it is the so-called embedding that reduces the covariant representation in terms of the corresponding canonical ones (cf. Remark 2.11). As reference spaces of the CAR- and CCR-algebras we will use in this paper the space of solutions of the Weyl- and the $\mathfrak{F}$-Equation introduced in Subsections 2.7 and 2.8. respectively, and will therefore call the corresponding already typical nets of local $\mathrm{C}^{*}$ algebras Weyl- resp. F-net. Some relations of the latter with the vector potential will also be mentioned. We will also relate the present construction to the nets specified in 48, since in this reference the nets were given without mentioning explicitly the corresponding relativistic wave equations. Concretely, we will show that the nets associated to the Weyl- and the $\mathfrak{F}$ Equation are isomorphic to the nets constructed in [48, cases $n=1$ and $n=2$ ], respectively. This isomorphy may then be easily generalised to arbitrary $n$. Thus producing the same net of local $\mathrm{C}^{*}$-algebras the generalisation of the Weyl- and the $\mathfrak{F}$-net constructed in the following will present a new methodological aspect w.r.t. [12, 48, namely we will show explicitly the relation to the corresponding relativistic wave equations. In particular the embeddings used here are different from those in [12, 48, and to construct them we will make essential use of distinguished elements of certain intertwiner spaces associated to representations of the little group $\mathcal{E}(2)$. By means of these elements the embedding will map any (vector-valued) test function into the space of solutions of the corresponding massless relativistic wave equation. This procedure illuminates another aspect of the reduction of the (fibre) degrees of freedom that is necessary when considering massless representations of nontrivial helicity (cf. Remark 2.11).

Denote by $\mathcal{B}\left(\mathbb{R}^{4}\right)$ the family of open and bounded regions in Minkowski space partially ordered by inclusion ' $\subset$ '. $\left(\mathcal{B}\left(\mathbb{R}^{4}\right), \subset\right)$ is then a directed index set which is stable under the action of the Poincaré group [14, Sections 5.1 and 7.1].

3.1 Definition $A$ correspondence $\mathcal{B}\left(\mathbb{R}^{4}\right) \ni \mathcal{O} \mapsto \mathcal{A}(\mathcal{O})$ where the local algebras $\mathcal{A}(\mathcal{O})$ are (abstract) unital $\mathrm{C}^{*}$-algebras with common unit $\mathbb{1}$, is called a Haag-Kastler net (HK-net for short) if the following conditions are satisfied:

(i) (Isotony) If $\mathcal{O}_{1} \subseteq \mathcal{O}_{2}$, then $\mathcal{A}\left(\mathcal{O}_{1}\right) \subseteq \mathcal{A}\left(\mathcal{O}_{2}\right), \mathcal{O}_{1}, \mathcal{O}_{2} \in \mathcal{B}\left(\mathbb{R}^{4}\right)$. We denote by $\mathcal{A}:=\lim \mathcal{A}(\mathcal{O})$ the corresponding inductive limit which is called the quasi local algebra.

(ii) (Additivity) Let $\left\{\mathcal{O}_{\lambda}\right\}_{\lambda \in \Lambda} \subset \mathcal{B}\left(\mathbb{R}^{4}\right)$ with $\cup_{\lambda} \mathcal{O}_{\lambda} \in \mathcal{B}\left(\mathbb{R}^{4}\right)$. The net $\mathcal{O} \mapsto \mathcal{A}(\mathcal{O})$ satisfies additivity if for any such $\left\{\mathcal{O}_{\lambda}\right\}_{\lambda}$ the following equation holds in $\mathcal{A}: \mathcal{A}\left(\cup_{\lambda} \mathcal{O}_{\lambda}\right)=\mathrm{C}^{*}\left(\cup_{\lambda} \mathcal{A}\left(\mathcal{O}_{\lambda}\right)\right)$.

(iii) (Causality) For any $\mathcal{O}_{1} \in \mathcal{B}\left(\mathbb{R}^{4}\right)$ space-like separated w.r.t. $\mathcal{O}_{2} \in \mathcal{B}\left(\mathbb{R}^{4}\right)$ (we denote this by $\left.\mathcal{O}_{1} \perp \mathcal{O}_{2}\right)$, then $\mathcal{A}\left(\mathcal{O}_{1}\right)$ commutes elementwise with $\mathcal{A}\left(\mathcal{O}_{2}\right)$ in $\mathcal{A}$.

Suppose further that there exists a representation $\alpha_{(\cdot)}$ of the $\widetilde{\mathcal{P}_{+}^{\uparrow}}$ in terms of ${ }^{*}$-automorphisms of $\mathcal{A}$, i.e. $\widetilde{\mathcal{P}_{+}^{\uparrow}} \ni g \mapsto \alpha_{g} \in \operatorname{Aut} \mathcal{A}$. 
(iv) (Covariance) The net $\mathcal{O} \mapsto \mathcal{A}(\mathcal{O})$ transforms covariantly w.r.t. $\alpha$, if for every $\mathcal{O} \in \mathcal{B}\left(\mathbb{R}^{4}\right)$ we have $\alpha_{g}(\mathcal{A}(\mathcal{O}))=\mathcal{A}(g \mathcal{O}), g \in \widetilde{\mathcal{P}_{+}^{\uparrow}}$, where $g \mathcal{O}:=\{g x \mid x \in \mathcal{O}\}$.

Next we will introduce the notion of isomorphic HK-nets (cf. [22, Section 3]).

3.2 Definition Two HK-nets $\left(\mathcal{A}^{(\mathrm{i})}(\mathcal{O}), \alpha_{(\cdot)}^{(\mathrm{i})}\right)_{\mathcal{O} \in \mathcal{B}\left(\mathbb{R}^{4}\right)}$ with quasi local algebras $\mathcal{A}^{(i)}, i=1,2$, are called isomorphic if there exists a ${ }^{*}$-isomorphism $\Lambda: \mathcal{A}^{(1)} \rightarrow \mathcal{A}^{(2)}$ which preserves localisation, i.e. $\Lambda\left(\mathcal{A}^{(1)}(\mathcal{O})\right)=\mathcal{A}^{(2)}(\mathcal{O}), \mathcal{O} \in \mathcal{B}\left(\mathbb{R}^{4}\right)$, and intertwines between the corresponding actions of the Poincaré group, i.e. $\Lambda \alpha_{g}^{(1)}=\alpha_{g}^{(2)} \Lambda, g \in \widetilde{\mathcal{P}_{+}^{\uparrow}}$.

Following the strategy suggested in [14, Section 8.3] we will now study a subclass of HK-nets, namely those where the local $\mathrm{C}^{*}$-algebras are certain $\mathrm{C}^{*}$-subalgebras of the CAR- resp. CCRalgebras. Due to the nice functorial properties of these algebras it is possible to encode the axioms of isotony, additivity, causality and covariance of the HK-net at the level of the respective reference spaces. We will call the result of this construction a free net. (The indices $\mathrm{F} / \mathrm{B}$ below denote the Fermi/Bose cases.)

3.3 Definition We consider the following tuples associated to $\left(\mathbb{R}^{4}, \perp, \widetilde{\mathcal{P}_{+}^{\uparrow}}\right)$, where $\perp$ is the causal disjointness relation given by the Minkowski metric on $\mathbb{R}^{4}$.

(i) In the Fermi or CAR case we have $\left(h_{\mathrm{F}},\langle\cdot, \cdot\rangle, \Gamma, V_{\mathrm{F}}, \mathcal{T}_{\mathrm{F}}, T_{\mathrm{F}}, \mathfrak{I}_{\mathrm{F}}\right)$, where $\left(h_{\mathrm{F}},\langle\cdot, \cdot\rangle\right)$ is a complex Hilbert space and $\Gamma$ an anti-unitary involution on it. $V_{\mathrm{F}}$ denotes a unitary representation of $\widehat{\mathcal{P}_{+}^{\uparrow}}$ on $\left(h_{\mathrm{F}},\langle\cdot, \cdot\rangle\right)$. Further, $\mathcal{T}_{\mathrm{F}}$ is the set of test functions on $\mathbb{R}^{4}$ with compact support and $T_{\mathrm{F}}$ is a representation of $\widetilde{\mathcal{P}_{+}^{\uparrow}}$ on $\mathcal{T}_{\mathrm{F}}$ satisfying the following support property: if $f \in \mathcal{T}_{\mathrm{F}}$ with $\operatorname{supp} f \subset \mathcal{O} \in \mathcal{B}\left(\mathbb{R}^{4}\right)$, then $T_{\mathrm{F}}(g) f \in \mathcal{T}_{\mathrm{F}}$ with $\operatorname{supp} T_{\mathrm{F}}(g) f \subset g \mathcal{O}, g \in \widetilde{\mathcal{P}_{+}^{\uparrow}}$. Finally, we require for the linear embedding $\mathfrak{I}_{\mathrm{F}}: \mathcal{T}_{\mathrm{F}} \longrightarrow h_{\mathrm{F}}$ the following properties:

(F1) ( $\Gamma$-invariance.) For an arbitrary $f \in \mathcal{T}_{\mathrm{F}}$ with $\operatorname{supp} f \subset \mathcal{O} \in \mathcal{B}\left(\mathbb{R}^{4}\right)$, there exists a $k \in \mathcal{T}_{\mathrm{F}}$ such that $\Gamma \mathfrak{I}_{\mathrm{F}} f=\mathfrak{I}_{\mathrm{F}} k$ and $\operatorname{supp} k \subset \mathcal{O}$.

(F2) (Causality.) For all $f, k \in \mathcal{T}_{\mathrm{F}}$ such that $\operatorname{supp} f \perp \operatorname{supp} k$, we have $\left\langle\mathfrak{I}_{\mathrm{F}} f, \mathfrak{I}_{\mathrm{F}} k\right\rangle=0$.

(F3) (Covariance.) $\Gamma V_{\mathrm{F}}(g)=V_{\mathrm{F}}(g) \Gamma$ and $\mathfrak{I}_{\mathrm{F}} T_{\mathrm{F}}(g)=V_{\mathrm{F}}(g) \mathfrak{I}_{\mathrm{F}}$, for all $g \in \widetilde{\mathcal{P}_{+}^{\uparrow}}$.

(ii) In the Bose or CCR case we have $\left(h_{\mathrm{B}}, \sigma, V_{\mathrm{B}}, \mathcal{T}_{\mathrm{B}}, T_{\mathrm{B}}, \mathfrak{I}_{\mathrm{B}}\right)$, where $V_{\mathrm{B}}$ is a symplectic representation of $\widetilde{\mathcal{P}_{+}^{\uparrow}}$ on the real symplectic space $\left(h_{\mathrm{B}}, \sigma\right)$, i.e. $V_{\mathrm{B}}(g), g \in \widetilde{\mathcal{P}_{+}^{\uparrow}}$, is a bijection of $h_{\mathrm{B}}$ that leaves $\sigma$ invariant. $\mathcal{T}_{\mathrm{B}}$ is again the set of test functions on $\mathbb{R}^{4}$ with compact support and $T_{\mathrm{B}}$ is a representation of $\widetilde{\mathcal{P}_{+}^{\uparrow}}$ on $\mathcal{T}_{\mathrm{B}}$ satisfying the same support property as in the fermionic case. We require for the linear embedding $\mathfrak{I}_{\mathrm{B}}: \mathcal{T}_{\mathrm{B}} \longrightarrow h_{\mathrm{B}}$ the following properties:

(B1) (Causality.) For all $f, k \in \mathcal{T}_{\mathrm{B}}$ such that $\operatorname{supp} f \perp \operatorname{supp} k$, we have $\sigma\left(\mathfrak{I}_{\mathrm{B}} f, \mathfrak{I}_{\mathrm{B}} k\right)=0$.

(B2) (Covariance.) $\mathfrak{I}_{\mathrm{B}} T_{\mathrm{B}}(g)=V_{\mathrm{B}}(g) \mathfrak{I}_{\mathrm{B}}$, for all $g \in \widetilde{\mathcal{P}_{+}^{\uparrow}}$.

Next we will show that the tuples that were specified in the preceding definition characterise in a canonical way a HK-net.

3.4 Theorem Assume the notation given in Definition 3.3 and consider the following nets of local linear submanifolds of the corresponding reference spaces:

$$
\begin{aligned}
& \mathcal{B}\left(\mathbb{R}^{4}\right) \ni \mathcal{O} \longmapsto h_{\mathrm{F}}(\mathcal{O}):=\left\{\mathfrak{I}_{\mathrm{F}} f \mid f \in \mathcal{T}_{\mathrm{F}} \text { and } \quad \operatorname{supp} f \subset \mathcal{O}\right\} \subset h_{\mathrm{F}}, \\
& \mathcal{B}\left(\mathbb{R}^{4}\right) \ni \mathcal{O} \longmapsto h_{\mathrm{B}}(\mathcal{O}):=\left\{\mathfrak{I}_{\mathrm{B}} f \mid f \in \mathcal{T}_{\mathrm{B}} \text { and } \quad \operatorname{supp} f \subset \mathcal{O}\right\} \subset h_{\mathrm{B}} .
\end{aligned}
$$

Then we have 
(i) The net of local $\mathrm{C}^{*}$-algebras given by

$$
\mathcal{B}\left(\mathbb{R}^{4}\right) \ni \mathcal{O} \longmapsto \mathcal{A}_{\mathrm{F}}(\mathcal{O}):=\mathrm{C}^{*}\left(\left\{\mathfrak{a}(\varphi) \mid \varphi \in h_{\mathrm{F}}(\mathcal{O})\right\}\right)^{\mathbb{Z}_{2}} \subset \mathrm{CAR}\left(h_{\mathrm{F}}, \Gamma\right)
$$

is a HK-net. Here $\mathfrak{a}(\cdot)$ denote the generators of $\mathrm{CAR}\left(h_{\mathrm{F}}, \Gamma\right)$ and $\mathcal{A}^{\mathbb{Z}_{2}}$ means the fixed point subalgebra of the $C^{*}$-algebra $\mathcal{A}$ w.r.t. Bogoljubov automorphism associated to the unitarity $-\mathbb{1}$. The covariance of this net of local $C^{*}$-algebras is realised by the Bogoljubov automorphisms $\alpha_{g}$ associated to the Bogoljubov unitaries $V_{\mathrm{F}}(g), g \in \widetilde{\mathcal{P}_{+}^{\uparrow}}$.

(ii) The net of local $\mathrm{C}^{*}$-algebras given by

$$
\mathcal{B}\left(\mathbb{R}^{4}\right) \ni \mathcal{O} \longmapsto \mathcal{A}_{\mathrm{B}}(\mathcal{O}):=\mathrm{C}^{*}\left(\left\{W(\varphi) \mid \varphi \in h_{\mathrm{B}}(\mathcal{O})\right\}\right) \subset \operatorname{CCR}\left(h_{\mathrm{B}}, \sigma\right)
$$

is a HK-net. Here $W(\cdot)$ denote the Weyl elements (generators) of $\operatorname{CCR}\left(h_{\mathrm{B}}, \sigma\right)$. The covariance of the net is given by the Bogoljubov automorphisms $\alpha_{g}$ associated to $V_{\mathrm{B}}(g), g \in \widetilde{\mathcal{P}_{+}^{\uparrow}}$.

We call the nets of $C^{*}$-algebras given in (i) and (ii) above free nets.

Proof: First note that from the $\Gamma$-invariance property $(\mathrm{F} 1)$ we have that $\Gamma h_{\mathrm{F}}(\mathcal{O})=h_{\mathrm{F}}(\mathcal{O})$, $\mathcal{O} \in \mathcal{B}\left(\mathbb{R}^{4}\right)$, which implies $\mathcal{A}_{\mathrm{F}}(\mathcal{O})^{*}=\mathcal{A}_{\mathrm{F}}(\mathcal{O})$ (as a set). The isotony of the local $\mathrm{C}^{*}$-algebras in the CAR and the CCR case follows immediately from the isotony property of the corresponding nets of linear submanifolds $\mathcal{O} \mapsto h_{\mathrm{F} / \mathrm{B}}(\mathcal{O})$.

To prove additivity we will show first that for $\left\{\mathcal{O}_{\lambda}\right\}_{\lambda \in \Lambda} \subset \mathcal{B}\left(\mathbb{R}^{4}\right)$ as in Definition 3.1 (ii) we have for the nets of local linear submanifolds

$$
h_{\mathrm{F} / \mathrm{B}}\left(\cup_{\lambda} \mathcal{O}_{\lambda}\right)=\operatorname{span}\left\{h_{\mathrm{F} / \mathrm{B}}\left(\mathcal{O}_{\lambda}\right) \mid \lambda \in \Lambda\right\} .
$$

Indeed, the inclusion ' $\supseteq$ ' follows from the linearity of $\mathfrak{I}$ and the fact that if $\operatorname{supp} f_{\lambda_{l}} \subset \mathcal{O}_{\lambda_{l}}$, $l=1, \ldots, L$, then $\operatorname{supp}\left(\sum_{l} \mu_{\lambda_{l}} f_{\lambda_{l}}\right) \subset \cup_{\lambda_{l}} \mathcal{O}_{\lambda_{l}}$ for $f_{\lambda_{l}} \in \mathcal{T}_{\mathrm{F} / \mathrm{B}}$ and $\mu_{\lambda_{l}} \in \mathbb{C}$. To show the converse inclusion take $f \in \mathcal{T}_{\mathrm{F} / \mathrm{B}}$ with supp $f \subset \cup_{\lambda} \mathcal{O}_{\lambda}$. By compactness there exists a finite subcovering such that supp $f \subset \cup_{l=1}^{L} \mathcal{O}_{\lambda_{l}}$ and using a subordinate smooth partition of unity (which exists since $\mathbb{R}^{4}$ is paracompact) we can write $f=f_{\lambda_{1}}+\ldots+f_{\lambda_{L}}$, where $f_{\lambda_{l}} \in \mathcal{T}_{\mathrm{F} / \mathrm{B}}$ and $\operatorname{supp} f_{\lambda_{l}} \subset \mathcal{O}_{\lambda_{l}}$, $l=1, \ldots, L$. Therefore

$$
\mathfrak{I} f=\mathfrak{I} f_{\lambda_{1}}+\ldots+\Im f_{\lambda_{L}} \in \operatorname{span}\left\{h_{\mathrm{F} / \mathrm{B}}\left(\mathcal{O}_{\lambda}\right) \mid \lambda \in \Lambda\right\} .
$$

Now additivity follows from the properties of the generators of the CAR- and CCR-algebras, cf. [14, Section 8.3].

For the causality property take $\mathcal{O}_{1}, \mathcal{O}_{2} \in \mathcal{B}\left(\mathbb{R}^{4}\right)$ with $\mathcal{O}_{1} \perp \mathcal{O}_{2}$. Now in the CAR case define the sets $\mathfrak{P}_{i}$ of polynomials in the generators $\mathfrak{a}\left(\varphi_{i}\right), \varphi_{i} \in h_{\mathrm{F}}\left(\mathcal{O}_{i}\right), i=1,2$, such that the degree of the corresponding monomials is even. From property (F2) and the CAR's we have that $\mathfrak{a}\left(\varphi_{1}\right) \mathfrak{a}\left(\varphi_{2}\right)+\mathfrak{a}\left(\varphi_{2}\right) \mathfrak{a}\left(\varphi_{1}\right)=0$ for all $\varphi_{i} \in h_{\mathrm{F}}\left(\mathcal{O}_{i}\right), i=1,2$, and therefore $\left[\mathfrak{P}_{1}, \mathfrak{P}_{2}\right]=0$. Now, since $\mathfrak{P}_{i}$ is dense in $\mathcal{A}_{\mathrm{F}}\left(\mathcal{O}_{i}\right), i=1,2$, we obtain that $\left[\mathcal{A}_{\mathrm{F}}\left(\mathcal{O}_{1}\right), \mathcal{A}_{\mathrm{F}}\left(\mathcal{O}_{2}\right)\right]=0$ in $\mathcal{A}$. In the Bose case note that from (B2) and the Weyl relation we have $W\left(\varphi_{1}\right) W\left(\varphi_{2}\right)=W\left(\varphi_{2}\right) W\left(\varphi_{1}\right)$ for all $\varphi_{i} \in h_{\mathrm{B}}\left(\mathcal{O}_{i}\right)$ and since $\operatorname{span}\left\{W\left(\varphi_{i}\right) \mid \varphi_{i} \in h_{\mathrm{F}}\left(\mathcal{O}_{i}\right)\right\}$ is dense in $\mathcal{A}_{\mathrm{B}}\left(\mathcal{O}_{i}\right), i=1,2$, we also obtain in this case that $\left[\mathcal{A}_{\mathrm{B}}\left(\mathcal{O}_{1}\right), \mathcal{A}_{\mathrm{B}}\left(\mathcal{O}_{2}\right)\right]=0$ in $\mathcal{A}$.

Finally, to prove the covariance property denote by $\alpha_{g}^{(\mathrm{F})}$ resp. $\alpha_{g}^{(\mathrm{B})}$ the Bogoljubov automorphisms associated to $V_{\mathrm{F}}(g)$ resp. $V_{\mathrm{B}}(g), g \in \widetilde{\mathcal{P}_{+}^{\uparrow}}$. Now by the support properties of $T_{\mathrm{F}}(g)$ and $T_{\mathrm{B}}(g)$ as well as by $(\mathrm{F} 3)$ and $(\mathrm{B} 2)$ we have

$$
V_{\mathrm{F}}(g) h_{\mathrm{F}}(\mathcal{O})=h_{\mathrm{F}}(g \mathcal{O}) \quad \text { and } \quad V_{\mathrm{B}}(g) h_{\mathrm{B}}(\mathcal{O})=h_{\mathrm{B}}(g \mathcal{O}) .
$$


Now from the way the Bogoljubov automorphisms act on the corresponding generators of the CAR/CCR-algebras it follows from the preceding equations that

$$
\alpha_{g}^{(\mathrm{F})}\left(\mathcal{A}_{\mathrm{F}}(\mathcal{O})\right)=\mathcal{A}_{\mathrm{F}}(g \mathcal{O}) \quad \text { and } \quad \alpha_{g}^{(\mathrm{B})}\left(\mathcal{A}_{\mathrm{B}}(\mathcal{O})\right)=\mathcal{A}_{\mathrm{B}}(g \mathcal{O}), \quad g \in \widetilde{\mathcal{P}_{+}^{\uparrow}},
$$

which concludes the proof. (See further Theorem 3.6 in [48] for a detailed proof of the covariance property.)

We will need later on the notion of isomorphic free nets explicitly. The isomorphy can be transcribed in terms of the corresponding reference spaces:

3.5 Proposition (i) Consider two tuples $\left(h_{i}, \Gamma_{i},\langle\cdot, \cdot\rangle_{i}, V_{i}, \mathcal{T}, T, \mathfrak{I}_{i}\right), i=1,2$, over the same test function space and satisfying the properties of Definition 3.3 (i). Suppose that there exists a unitary linear mapping $\lambda_{\mathrm{F}}: h_{1} \rightarrow h_{2}$ (i.e. $\left\langle\lambda_{\mathrm{F}}(\varphi), \lambda_{\mathrm{F}}(\psi)\right\rangle_{2}=\langle\varphi, \psi\rangle_{1}, \varphi, \psi \in h_{1}$ ) satisfying

$$
\lambda_{\mathrm{F}} V_{1}(g)=V_{2}(g) \lambda_{\mathrm{F}}, \quad \lambda_{\mathrm{F}} \Gamma_{1}(g)=\Gamma_{2}(g) \lambda_{\mathrm{F}}, g \in \widetilde{\mathcal{P}_{+}^{\uparrow}}, \quad \text { and } \quad \lambda_{\mathrm{F}} \mathfrak{I}_{1}=\mathfrak{I}_{2} .
$$

Then the corresponding fermionic free nets are isomorphic.

(ii) Consider two tuples $\left(h_{i}, \sigma_{i}, V_{i}, \mathcal{T}, T, \mathfrak{I}_{i}\right), i=1,2$, over the same test function space and satisfying the properties of Definition 3.3 (ii). Suppose that there exists a (real) linear symplectic bijection $\lambda_{\mathrm{B}}: h_{1} \rightarrow h_{2}$ (i.e. $\left.\sigma_{2}\left(\lambda_{\mathrm{B}}(\varphi), \lambda_{\mathrm{B}}(\psi)\right)=\sigma_{1}(\varphi, \psi), \varphi, \psi \in h_{1}\right)$ satisfying

$$
\lambda_{\mathrm{B}} V_{1}(g)=V_{2}(g) \lambda_{\mathrm{B}}, g \in \widetilde{\mathcal{P}_{+}^{\uparrow}}, \quad \text { and } \quad \lambda_{\mathrm{B}} \mathfrak{I}_{1}=\mathfrak{I}_{2} .
$$

Then the corresponding bosonic free nets are isomorphic.

Proof: The proof of (ii) is typical: denote by $W\left(\varphi_{i}\right), \varphi_{i} \in h_{i}$, the Weyl elements of the corresponding $\mathrm{C}^{*}$-algebras $\operatorname{CCR}\left(h_{i}, \sigma_{i}\right), i=1,2$. Then the mapping $\Lambda\left(W\left(\varphi_{1}\right)\right):=W\left(\lambda\left(\varphi_{1}\right)\right), \varphi_{1} \in h_{1}$, extends uniquely to an isomorphism (also denoted by $\Lambda$ ) of the corresponding CCR-algebras. Further, the equation $\lambda_{\mathrm{B}} \mathfrak{I}_{1}=\mathfrak{I}_{2}$ implies $\lambda_{\mathrm{B}} h_{1}(\mathcal{O})=h_{2}(\mathcal{O})$, so that for the local $\mathrm{C}^{*}$-subalgebras we have $\Lambda\left(\mathcal{A}_{1}(\mathcal{O})\right)=\mathcal{A}_{2}(\mathcal{O}), \mathcal{O} \in \mathcal{B}\left(\mathbb{R}^{4}\right)$. Finally, the intertwining property of $\lambda$ in Eq. (55) implies that $\Lambda \alpha_{g}^{(1)}=\alpha_{g}^{(2)} \Lambda, g \in \widetilde{\mathcal{P}_{+}^{\uparrow}}$.

3.6 Remark Part (ii) of Definition 3.3 contains some aspects of Segal's notion of quantisation for bosonic systems (cf. 61, p. 750], [8, p. 106]). With Definition 3.3 and concretely through the requirements on the embedding $\mathfrak{I}$ we incorporate to this program the axioms of local quantum physics. Note nevertheless that since Haag-Kastler axioms are stated in terms of abstract $\mathrm{C}^{*}$ algebras we do not require initially (in contrast with Segal's approach) that the abstract CCRalgebra is represented in any Hilbert space nor the specification of any regular state. (For further reasons on this last point see also [31, 32.)

We consider next also the spectrality condition, which in the context of free nets can be stated in terms of the tuples considered in Definition 3.3 .

3.7 Definition With the notation of Definition 3.3 we require respectively:

(F4) There exists a basis projection $P$ on $h_{\mathrm{F}}$ (i.e. an orthoprojection satisfying $P+\Gamma P \Gamma=$ $\mathbb{1})$ reducing the representation $V_{\mathrm{F}}$, i.e. $P V_{\mathrm{F}}(g)=V_{\mathrm{F}}(g) P, g \in \widetilde{\mathcal{P}_{+}^{\uparrow}}$, and such that the corresponding representation on $P h_{\mathrm{F}}$ is strongly continuous and satisfies the spectrality condition (i.e. the spectrum of the corresponding generators of the space-time translations is contained in the forward light cone $\overline{\mathcal{V}}_{+}$). 
(B3) There exists a real scalar product $s$ on $h_{\mathrm{B}}$ and an internal complexification $J$ satisfying $J^{2}=-\mathbb{1}, \sigma(\varphi, J \psi)=-\sigma(J \varphi, \psi), \sigma(\varphi, J \varphi)=s(\varphi, \varphi)$ and $|\sigma(\varphi, \psi)|^{2} \leq s(\varphi, \varphi) s(\psi, \psi)$, $\varphi, \psi \in h_{\mathrm{B}}$. W.r.t. this complexification $V_{\mathrm{F}}$ is a strongly continuous unitary representation on the one particle Hilbert space $\left(h_{\mathrm{B}}, k_{J}\right)$ satisfying the spectrality condition. Here $k_{J}=s+i \sigma$ denotes the corresponding complex scalar product.

3.8 Remark Recall first that the basis projection $P$ resp. the complexification $J$ characterise Fock states of the CAR- resp. CCR-algebras. We will show in this remark that the preceding definition implies the existence of a covariant representation of the $\mathrm{C}^{*}$-dynamical systems $\left(\mathrm{CAR}\left(h_{\mathrm{F}}, \Gamma\right), \mathbb{R}^{4}, \alpha_{(\cdot)}\right)$ and $\left(\mathrm{CCR}\left(h_{\mathrm{B}}, \sigma\right), \mathbb{R}^{4}, \alpha_{(\cdot)}\right)$ satisfying the spectrality condition (cf. [16] and [12, Teorems A.4.2 and A.4.5]). Compare also with the notion of covariant representations introduced in [57, 43].

(i) From (F4) and from standard results of the CAR theory [5] it can be shown that the Bogoljubov automorphisms $\alpha_{g}$, corresponding to $V_{\mathrm{F}}(g), g \in \widehat{\mathcal{P}_{+}^{\uparrow}}$, are uniquely implemented by unitary operators $Q_{g}$ on $\mathcal{F}_{a}\left(P h_{\mathrm{F}}\right)$ (the antisymmetric Fock space over $\left.P h_{\mathrm{F}}\right)$ that leave the Fock vacuum $\Omega$ invariant. Now it is straightforward calculation to show that on the set of finite particle vectors (which is dense in $\mathcal{F}_{a}\left(P h_{\mathrm{F}}\right)$ ) the following equations hold for all $\varphi \in h_{\mathrm{F}}$ and $g \in \widetilde{\mathcal{P}_{+}^{\uparrow}}$ :

$$
\pi_{P}\left(\alpha_{g}(\mathfrak{a}(\varphi))\right)=Q\left(P V_{\mathrm{F}}(g)\right) \pi_{P}(\mathfrak{a}(\varphi)) Q\left(P V_{\mathrm{F}}(g)\right)^{-1} \quad \text { and } \quad Q\left(P V_{\mathrm{F}}(g)\right) \Omega=\Omega,
$$

where $\pi_{P}$ is the Fock representation characterised by $P$ and $Q\left(P V_{\mathrm{F}}(g)\right)$ denotes the second quantisation of the corresponding subrepresentation on $\mathcal{F}_{a}\left(P h_{\mathrm{F}}\right)$. This implies that $Q_{g}=$ $Q\left(P V_{\mathrm{F}}(g)\right)$ and since $P V_{\mathrm{F}}(g)$ satisfies the spectrality condition on $P h_{\mathrm{F}}, Q\left(P V_{\mathrm{F}}(g)\right)$ will also satisfy it on $\mathcal{F}_{a}\left(P h_{\mathrm{F}}\right)$ [5].

(ii) In the CCR-case we obtain from (B3) and from the definition of the generating functional $h_{\mathrm{F}} \ni \varphi \rightarrow e^{-\frac{1}{4} s(\varphi, \varphi)}$ that the Bogoljubov automorphisms $\alpha_{g}$, corresponding to $V_{\mathrm{B}}(g), g \in$ $\widetilde{\mathcal{P}_{+}^{\uparrow}}$, are uniquely implemented by unitary operators $Q_{g}$ on $\mathcal{F}_{s}\left(h_{\mathrm{B}}\right)$ (the symmetric Fock space over the one particle Hilbert space $h_{\mathrm{B}}$ ) that leave the Fock vacuum $\Omega$ invariant (see 14. Section 8.2]). But again a straightforward calculation shows that on the set of coherent vectors (which is total in $\mathcal{F}_{s}\left(h_{\mathrm{B}}\right)$ cf. [33. Chapter 2]) the following equations hold for all $\varphi \in h_{\mathrm{B}}$ and $g \in \widetilde{\mathcal{P}_{+}^{\uparrow}}$ :

$$
\pi_{J}\left(\alpha_{g}(W(\varphi))\right)=Q\left(V_{\mathrm{B}}(g)\right) \pi_{J}(W(\varphi)) Q\left(V_{\mathrm{B}}(g)\right)^{-1} \quad \text { and } \quad Q\left(V_{\mathrm{B}}(g)\right) \Omega=\Omega,
$$

where $\pi_{J}$ is the Fock representation characterised by $J$ and $Q\left(V_{\mathrm{B}}(g)\right)$ denotes the second quantisation of $V_{\mathrm{B}}$ on $\mathcal{F}_{s}\left(h_{\mathrm{B}}\right)$. This shows that $Q_{g}=Q\left(V_{\mathrm{B}}(g)\right)$. Now by the property of Fock states (cf. [14, Section 8.2.7]) that any positive operator on $\left(h, k_{J}\right)$ has a positive second quantisation on $\mathcal{F}_{s}\left(h_{\mathrm{B}}\right)$, we get finally that the spectrality condition of $V_{\mathrm{B}}(g)$ on the one particle Hilbert space implies the spectrality condition for $Q\left(V_{\mathrm{B}}(g)\right)$ on $\mathcal{F}_{s}\left(h_{\mathrm{B}}\right)$.

3.9 Remark The existence of the structures given in Definition 3.3(i) or (ii) satisfying (F1) - (F4) or (B1) - (B3) (and therefore the existence of free nets) is shown in the context of Minkowski space in [48, 12]. In this paper we construct free nets of local $\mathrm{C}^{*}$-algebras associated to massive (massless) systems with arbitrary spin (helicity) [77. The embedding, which is the central object of the free net construction, is given for example in the massive case of [12] as a direct sum of those mappings that reduce the covariant representation into the irreducible massive canonical representation (cf. also Remark 2.5). In other words the embedding selects from the 
algebraically reducible covariant representation two irreducible components. ${ }^{6}$ Summing up, we have transcribed Haag-Kastler's axioms in terms of the embeddings $\mathfrak{I}$ and given a neat group theoretical interpretation of it in the context of the Poincaré group. Note finally that the free net construction avoids (in the spirit of local quantum physics) any explicit use of the notion of quantum field.

We finish this section adapting Lemma A.1.4 in [12] to the present massless case. This result will be essential for proving the causality property of the following models of free nets.

3.10 Lemma Let $x \in \mathbb{R}^{4}$ be a spacelike vector and $\beta_{n}(\cdot)$ a matrix-valued function on $\mathcal{C}_{+}$such that at each point $p \in \mathcal{C}_{+}$the matrix elements of $\beta_{n}(p)$ are homogeneous polynomials of degree $n$ in $p_{\mu}, \mu=0,1,2,3$. Then we have

$$
\begin{aligned}
& \int_{\mathcal{C}_{+}}\left(e^{i p x}-e^{-i p x}\right) \beta_{n}(p) \mu_{0}(\mathrm{~d} p)=0, \quad n \text { even }, \\
& \int_{\mathcal{C}_{+}}\left(e^{i p x}+e^{-i p x}\right) \beta_{n}(p) \mu_{0}(\mathrm{~d} p)=0, \quad n \text { odd } .
\end{aligned}
$$

Proof: It is well-known that for $x^{2}<0$ the Pauli-Jordan function

$$
\Delta(x)=\int_{\mathcal{C}_{+}} e^{-i p x} \mu_{0}(\mathrm{~d} p)
$$

is an even $\mathrm{C}^{\infty}$ function, i.e. $\Delta(x)=\Delta(-x)$ (see [58, pgs. 71 and 107]). Let $\alpha$ be a multi-index and $|\alpha|:=\alpha_{0}+\alpha_{1}+\alpha_{2}+\alpha_{3}$. Then

$$
\left.\frac{\partial^{|\alpha|} \Delta}{\partial x^{\alpha}}\right|_{x}=(-i)^{|\alpha|} \int_{\mathcal{C}_{+}} p_{\alpha} e^{-i p x} \mu_{0}(\mathrm{~d} p) \text { is an }\left\{\begin{array}{l}
\text { even function, if }|\alpha| \text { is even. } \\
\text { odd function, if }|\alpha| \text { is odd. }
\end{array}\right.
$$

But from hypothesis the matrix elements of $\beta_{n}(p)$ are homogeneous polynomials in $p_{\mu}$ of degree $n$ and therefore the last expression implies Eqs. (56) and (57).

We will denote the objects of the constructions in the following Sections with the subindex $\mathfrak{W}$ or $\mathfrak{F}$ depending if the net is associated to the Weyl Equation or to the $\mathfrak{F}$-Equation, respectively. All the mentioned models will have a particular function $\beta(\cdot)$ (recall the preceding lemma) that characterises the corresponding scalar products and symplectic forms.

\subsection{Weyl net}

The following construction will illustrate the fermionic axioms (F1) - (F4) making explicit use of the Weyl equation. Further it will provide the simplest nontrivial example where certain intertwiner spaces are explicitly introduced in order to define the corresponding embeddings that satisfy the conditions already stated in Subsection 2.6. Indeed, making use of the notation and results of Subsection 2.7 and of the particular structure of the intertwiner space associated to the finite-dimensional representations $D^{\left(\frac{1}{2}, 0\right)}$ and $D^{\left(0, \frac{1}{2}\right)}$ restricted to the massless little group $\mathcal{E}(2)$, we will construct the free net associated to the Weyl equation. The free net resulting from this construction is isomorphic to the one given in [4] for $n=1$ (cf. Remark 3.17 (i)).

\footnotetext{
${ }^{6}$ These types of embeddings play also an important role in the (rigorous) context of quantised fields, defined mathematically as operator-valued distributions (see e.g. [58] Theorem X.42] or [8] Appendix B] in the example of the Klein-Gordon field).
} 
We will see later on in the section that this construction procedure can be easily adapted to the bosonic case. Recall that given two representations $V, V^{\prime}$ of a group $\mathcal{G}$ on finite dimensional Hilbert spaces $\mathcal{H}, \mathcal{H}^{\prime}$ the corresponding intertwiner space is defined as

$$
\left(V(\mathcal{G}), V^{\prime}(\mathcal{G})\right):=\left\{\Psi: \mathcal{H} \rightarrow \mathcal{H}^{\prime} \mid \psi \text { is linear and } \Psi V(g)=V^{\prime}(g) \Psi, g \in \mathcal{G}\right\} .
$$

3.11 Lemma With the notion above we compute the following intertwiner spaces:

$$
\begin{aligned}
& \left(D^{\left(\frac{1}{2}, 0\right)}(\mathcal{E}(2)), D^{\left(0, \frac{1}{2}\right)}(\mathcal{E}(2))\right)=\left\{\left(\begin{array}{ll}
0 & s \\
0 & 0
\end{array}\right) \mid s \in \mathbb{R}\right\}, \\
& \left(D^{\left(\frac{1}{2}, 0\right)}(\mathcal{E}(2)), D^{\left(\frac{1}{2}, 0\right)}(\mathcal{E}(2))\right)=\mathbb{C} \mathbb{1} .
\end{aligned}
$$

Proof: The first intertwiner space consists of all $M \in \operatorname{Mat}_{2}(\mathbb{R})$ such that $M L=\bar{L} M$ for all $L \in \mathcal{E}(2)$. It is now immediate to check that $M=\left(\begin{array}{ll}0 & s \\ 0 & 0\end{array}\right), s \in \mathbb{R}$. The triviality of the second intertwiner space is a straightforward computation.

\subsubsection{CAR-algebra}

First recall the definitions associated to the Weyl Equation given in Subsection 2.7 and the form of the $H_{p}$-matrices, $p \in \mathcal{C}_{+}^{\circ}$, given in Eq. (21). We consider the complex Hilbert space $h_{\mathfrak{W}}:=h_{+} \oplus h_{-} \oplus h_{+} \oplus h_{-}$with the scalar product given by $\langle\cdot, \cdot\rangle_{\mathfrak{W}}:=\langle\cdot, \cdot\rangle_{+} \oplus\langle\cdot, \cdot\rangle_{-} \oplus\langle\cdot, \cdot\rangle_{+} \oplus\langle\cdot, \cdot\rangle_{-}$. To define the anti-linear involution on $h_{\mathfrak{w}}$ consider first the mapping $\Gamma_{1}: h_{+} \longrightarrow h_{-}$given by

$$
\left(\Gamma_{1} \varphi_{+}\right)(p):=\overline{H_{p}} \Gamma_{0} H_{p}^{-1} \varphi_{+}(p)=\overline{H_{p}} \Gamma_{0}\left(\begin{array}{c}
\chi_{+}(p) \\
0
\end{array}\right),
$$

where $\varphi_{+} \in h_{+}$and $\Gamma_{0}: \mathcal{H}^{\left(\frac{1}{2}, 0\right)} \rightarrow \mathcal{H}^{\left(0, \frac{1}{2}\right)}$ is an anti-unitary involution (conjugation). It can be easily shown that $\Gamma_{1}$ is anti-linear and that it satisfies the equation $\left\langle\Gamma_{1} \varphi_{+}, \Gamma_{1} \psi_{+}\right\rangle_{-}=\left\langle\psi_{+}, \varphi_{+}\right\rangle_{+}$ for all $\varphi_{+}, \psi_{+} \in h_{+}$.

Finally, define in terms of $\Gamma_{1}$ the anti-unitary involution on $h_{\mathfrak{W}}$ as

$$
\Gamma_{\mathfrak{W}}\left(\varphi_{+} \oplus \varphi_{-} \oplus \psi_{+} \oplus \psi_{-}\right):=\Gamma_{1}^{-1} \psi_{-} \oplus \Gamma_{1} \psi_{+} \oplus \Gamma_{1}^{-1} \varphi_{-} \oplus \Gamma_{1} \varphi_{+} .
$$

$\Gamma_{\mathfrak{W}}$ is anti-linear and it can easily be checked that,

$$
\Gamma_{\mathfrak{W}}^{2}=\mathbb{1} \quad \text { and } \quad\left\langle\Gamma_{\mathfrak{W}} \varphi^{(1)}, \Gamma_{\mathfrak{W}} \varphi^{(2)}\right\rangle_{\mathfrak{W}}=\left\langle\varphi^{(2)}, \varphi^{(1)}\right\rangle_{\mathfrak{W}}
$$

$\varphi^{(i)} \in h_{\mathfrak{W}}, i=1,2$. The $\mathrm{C}^{*}$-algebra $\operatorname{CAR}\left(h_{\mathfrak{W}}, \Gamma_{\mathfrak{W}}\right)$ is therefore uniquely given.

\subsubsection{Existence theorem for the local algebras}

We consider here on the test function space spaces $\mathcal{T}_{\mathfrak{W}}:=\mathcal{S}\left(\mathbb{R}^{4}, \mathcal{H}^{\left(0, \frac{1}{2}\right)}\right), h_{+}$and $h_{-}$the following covariant and canonical representations of $\widetilde{\mathcal{P}_{+}^{\uparrow}}=\operatorname{SL}(2, \mathbb{C}) \ltimes \mathbb{R}^{4} \ni g=(A, a)$ : for $f \in \mathcal{T}_{\mathfrak{W}}, \varphi_{ \pm} \in h_{ \pm}$

$$
\begin{aligned}
& \left(T_{\mathfrak{W}}(g) f\right)(x):=\bar{A} f\left(\Lambda_{A}^{-1}(x-a)\right), \\
& \left(V_{1}(g) \varphi_{+}\right)(p):=e^{-i p a} A \varphi_{+}\left(\Lambda_{A}^{-1} p\right), \quad\left(V_{3}(g) \varphi_{+}\right)(p):=e^{i p a} A \varphi_{+}\left(\Lambda_{A}^{-1} p\right), \\
& \left(V_{2}(g) \varphi_{-}\right)(p):=e^{-i p a} \bar{A} \varphi_{-}\left(\Lambda_{A}^{-1} p\right), \quad\left(V_{4}(g) \varphi_{-}\right)(p):=e^{i p a} \bar{A} \varphi_{-}\left(\Lambda_{A}^{-1} p\right) .
\end{aligned}
$$

Note that the covariant representation $T_{\mathfrak{W}}$, satisfies the support property mentioned in part (i) of Definition 3.3. We consider next the following reducible representation of $\widetilde{\mathcal{P}_{+}^{\uparrow}}$ over $h_{\mathfrak{W}}$ :

$$
V_{\mathfrak{W}}:=V_{1} \oplus V_{2} \oplus V_{3} \oplus V_{4}
$$


3.12 Lemma The equation $\Gamma_{\mathfrak{W}} V_{\mathfrak{W}}(g)=V_{\mathfrak{W}}(g) \Gamma_{\mathfrak{W}}$ holds for all $g \in \widetilde{\mathcal{P}_{+}^{\uparrow}}$.

Proof: The equation is based on the following intertwining properties of $\Gamma_{1}$ :

$$
\left.\begin{array}{l}
\Gamma_{1} V_{1}(g)=V_{4}(g) \Gamma_{1} \\
\Gamma_{1} V_{3}(g)=V_{2}(g) \Gamma_{1}
\end{array}\right\}
$$

which are a direct consequence of the definitions.

Further we consider the embeddings $\mathfrak{I}_{1,3}: \mathcal{T}_{\mathfrak{W}} \longrightarrow h_{+}$and $\mathfrak{I}_{2,4}: \mathcal{T}_{\mathfrak{W}} \longrightarrow h_{-}$defined for all $f \in \mathcal{T}_{\mathfrak{W}}$ by,

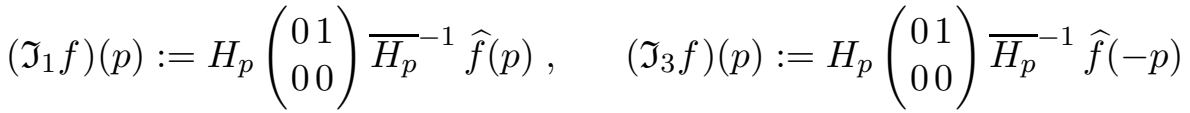

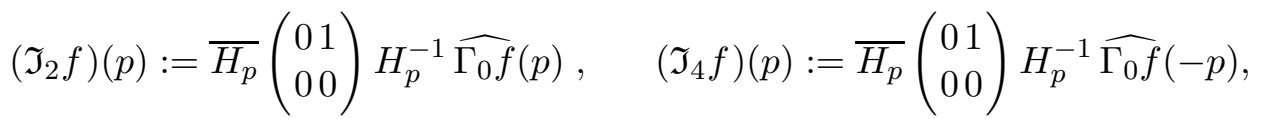

where the 'hat' $\widehat{f}$ means the Fourier transformation and $p$ is restricted to $\mathcal{C}_{+}^{\circ}$. Note that since $\left(D^{\left(0, \frac{1}{2}\right)}(\mathcal{E}(2)), D^{\left(\frac{1}{2}, 0\right)}(\mathcal{E}(2))\right) \ni\left(\begin{array}{l}0 \\ 0 \\ 0\end{array}\right) \in\left(D^{\left(\frac{1}{2}, 0\right)}(\mathcal{E}(2)), D^{\left(0, \frac{1}{2}\right)}(\mathcal{E}(2))\right)$ the above definitions are consistent.

Finally, the embedding that specifies the net structure is given by

$$
\mathfrak{I}_{\mathfrak{W}}: \mathcal{T}_{\mathfrak{W}} \longrightarrow h_{\mathfrak{W}}, \quad \mathfrak{I}_{\mathfrak{W}} f:=\mathfrak{I}_{1} f \oplus \mathfrak{I}_{2} f \oplus \mathfrak{I}_{3} f \oplus \mathfrak{I}_{4} f .
$$

3.13 Remark Note for instance that

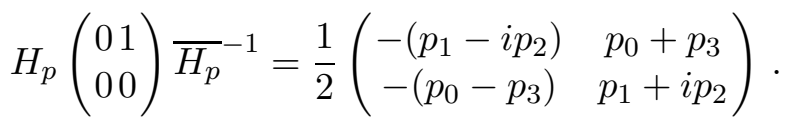

Therefore, the matrix elements of the previous expression correspond on position space to differential operators and $\mathfrak{I}_{\mathfrak{W}}$ will not change the localisation properties of $f$. Further, the embeddings (say $\mathfrak{I}_{1}$ ) that specify $\mathfrak{I}_{\mathfrak{W}}$ can be written in components as

$$
\left(\Im_{1} f\right)^{C}(p)=\left(H_{p}\right)_{B}^{C} Q^{B C^{\prime}} \varepsilon_{C^{\prime} B^{\prime}}\left({\overline{H_{p}}}^{-1}\right)_{E^{\prime}}^{B^{\prime}} \widehat{f^{E^{\prime}}}(p) \quad \text { (sum over repeated indices ), }
$$

where $\left(\varepsilon_{C^{\prime} B^{\prime}}\right):=\left(\begin{array}{rr}0 & 1 \\ -1 & 0\end{array}\right)$ and $\left(Q^{B C^{\prime}}\right):=\left(\begin{array}{ll}1 & 0 \\ 0 & 0\end{array}\right)$ is the matrix corresponding to the point $\frac{1}{2}(1,0,0,1)$ in the positive light cone (recall Subsection 2.4). Moreover, $Q$ can be seen as the 1-dimensional projection characterising the Weyl equation (see Subsection 2.7).

The covariance property of the net characterised by the preceding embedding is guaranteed by the following result

3.14 Lemma The equation, $\mathfrak{I}_{\mathfrak{W}} T_{\mathfrak{W}}(g)=V_{\mathfrak{W}}(g) \mathfrak{I}_{\mathfrak{W}}$, holds for all $g \in \widetilde{\mathcal{P}_{+}^{\uparrow}}$.

Proof: First recall that for $g=(A, a) \in \mathrm{SL}(2, \mathbb{C}) \ltimes \mathbb{R}^{4}, p \in \mathcal{C}_{+}^{\circ}$ and $q:=\Lambda_{A}^{-1} p$ the matrix $H_{p}^{-1} A H_{q} \in \mathcal{E}(2)$. Thus by Lemma 3.11 we have

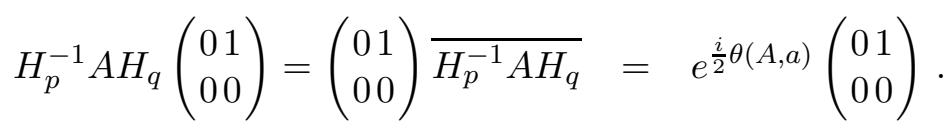


From this, the relations

$$
\left.\begin{array}{rl}
\mathfrak{I}_{1,3} T_{\mathfrak{W}}(g) & =V_{1,3}(g) \mathfrak{I}_{1,3} \\
\mathfrak{I}_{2,4} \Gamma_{0} T_{\mathfrak{W}}(g) & =V_{2,4}(g) \mathfrak{I}_{2,4} \Gamma_{0},
\end{array}\right\}
$$

can be easily shown and the intertwining equation of the statement is proved.

The next result will ensure causality for the net characterised by the embedding $\mathfrak{I}_{\mathfrak{W}}$.

3.15 Lemma If $\operatorname{supp} f \perp \operatorname{supp} k$ for $f, k \in \mathcal{T}_{\mathfrak{W}}$, then the equation $\left\langle\mathfrak{I}_{\mathfrak{W}} f, \mathfrak{I}_{\mathfrak{W}} k\right\rangle_{\mathfrak{W}}=0$ holds.

Proof: First put $\widetilde{\beta_{+}}(p):=\left(H_{p}^{-1}\right)^{*} H_{p}^{-1}$ and $\widetilde{\beta_{-}}(p)=\Gamma_{0} \widetilde{\beta_{+}}(p) \Gamma_{0}, p \in \mathcal{C}_{+}^{\circ}$ (see also Remark 2.12). Then we compute

$$
\begin{aligned}
\left\langle\mathfrak{I}_{\mathfrak{W}} f, \mathfrak{I}_{\mathfrak{W}} k\right\rangle_{\mathfrak{W}} & \\
= & \int_{\mathcal{C}_{+}}\left\langle\left(\mathfrak{I}_{1} f\right)(p), \widetilde{\beta_{+}}(p)\left(\mathfrak{I}_{1} k\right)(p)\right\rangle_{\mathbb{C}^{2}} \mu_{0}(\mathrm{~d} p)+\int_{\mathcal{C}_{+}}\left\langle\left(\mathfrak{I}_{2} \Gamma_{0} f\right)(p), \widetilde{\beta_{-}}(p)\left(\mathfrak{I}_{2} \Gamma_{0} k\right)(p)\right\rangle_{\mathbb{C}^{2}} \mu_{0}(\mathrm{~d} p) \\
& +\int_{\mathcal{C}_{+}}\left\langle\left(\mathfrak{I}_{3} f\right)(p), \widetilde{\beta_{+}}(p)\left(\mathfrak{I}_{3} k\right)(p)\right\rangle_{\mathbb{C}^{2}} \mu_{0}(\mathrm{~d} p)+\int_{+}\left\langle\left(\mathfrak{I}_{4} \Gamma_{0} f\right)(p), \widetilde{\beta_{-}}(p)\left(\mathfrak{I}_{4} \Gamma_{0} k\right)(p)\right\rangle_{\mathbb{C}^{2}} \mu_{0}(\mathrm{~d} p) \\
= & \int_{\mathcal{C}_{+}}\left\langle\widehat{f}(p), \beta_{+}(p) \widehat{k}(p)\right\rangle_{\mathbb{C}^{2}} \mu_{0}(\mathrm{~d} p)+\int_{+}\left\langle\widehat{\Gamma_{0} f}(p), \beta_{-}(p) \widehat{\Gamma_{0} k}(p)\right\rangle_{\mathbb{C}^{2}} \mu_{0}(\mathrm{~d} p) \\
& +\int_{\mathcal{C}_{+}}\left\langle\widehat{f}(-p), \beta_{+}(p) \widehat{k}(-p)\right\rangle_{\mathbb{C}^{2}} \mu_{0}(\mathrm{~d} p)+\int_{\mathcal{C}_{+}}\left\langle\widehat{\Gamma_{0} f}(-p), \beta_{-}(p) \widehat{\Gamma_{0} k}(-p)\right\rangle_{\mathbb{C}^{2}} \mu_{0}(\mathrm{~d} p) \\
= & \int_{\mathcal{C}_{+}}\left\langle\widehat{f}(p), \beta_{+}(p) \widehat{k}(p)\right\rangle_{\mathbb{C}^{2}} \mu_{0}(\mathrm{~d} p)+\int_{\mathcal{C}_{+}}\left\langle\widehat{k}(-p), \beta_{+}(p) \widehat{f}(-p)\right\rangle_{\mathbb{C}^{2}} \mu_{0}(\mathrm{~d} p) \\
& +\int_{\mathcal{C}_{+}}\left\langle\widehat{f}(-p), \beta_{+}(p) \widehat{k}(-p)\right\rangle_{\mathbb{C}^{2}} \mu_{0}(\mathrm{~d} p)+\int_{\mathcal{C}_{+}}\left\langle\widehat{k}(p), \beta_{+}(p) \widehat{f}(p)\right\rangle_{\mathbb{C}^{2}} \mu_{0}(\mathrm{~d} p) \\
= & 0,
\end{aligned}
$$

where $\beta_{+}(p):=\left({\overline{H_{p}}}^{-1}\right)^{*}\left(\begin{array}{ll}0 & 0 \\ 0 & 1\end{array}\right){\overline{H_{p}}}^{-1}=\overline{P^{\dagger}}$ and $\beta_{-}(p):=\Gamma_{0} \beta_{+}(p) \Gamma_{0}$ (recall Remark 2.13). The last equation follows from Lemma 3.10 and the fact that the matrix elements of $P^{\dagger}$ are homogeneous polynomials of degree 1 .

We can now prove the existence of a free net associated to the Weyl Equation, which we call Weyl net for short.

3.16 Theorem Consider the net of local linear submanifolds of $h_{\mathfrak{W}}$ given for $\mathcal{O} \in \mathcal{B}\left(\mathbb{R}^{4}\right)$ by

$$
\mathbb{R}^{4} \supset \mathcal{O} \longmapsto h_{\mathfrak{W}}(\mathcal{O}):=\left\{\mathfrak{I}_{\mathfrak{W}} f \mid f \in \mathrm{C}_{0}^{\infty}\left(\mathbb{R}^{4}, \mathcal{H}^{\left(0, \frac{1}{2}\right)}\right), \operatorname{supp} f \subset \mathcal{O}\right\}
$$

Then the net of local $C^{*}$-algebras defined by

$$
\mathbb{R}^{4} \supset \mathcal{O} \longmapsto \mathcal{A}_{\mathfrak{W}}(\mathcal{O}):=\mathrm{C}^{*}\left(\left\{\mathfrak{a}(\varphi) \mid \varphi \in h_{\mathfrak{W}}(\mathcal{O})\right\}\right)^{\mathbb{Z}_{2}},
$$

where the $\mathfrak{a}(\cdot)$ 's denote the generators of the $C^{*}$-algebra $\mathrm{CAR}\left(h_{\mathfrak{W}}, \Gamma_{\mathfrak{W}}\right)$, is a HK-net. 
Proof: First note that the local linear submanifolds satisfy the $\Gamma_{\mathfrak{W}^{-}}$-invariance property (F1) in Definition 3.3. Indeed, from the relations $\Gamma_{1}\left(\mathfrak{I}_{1} f\right)=\mathfrak{I}_{4}\left(\Gamma_{0} f\right)$ and $\Gamma_{1}\left(\mathfrak{I}_{3} f\right)=\mathfrak{I}_{2}\left(\Gamma_{0} f\right), f \in T_{\mathfrak{W}}$, it follows that $\Gamma_{\mathfrak{W}} \mathfrak{I}_{\mathfrak{W}} f=\mathfrak{I}_{\mathfrak{W}} f$ (which for the generators implies $\mathfrak{a}\left(\mathfrak{I}_{\mathfrak{W}} f\right)^{*}=\mathfrak{a}\left(\mathfrak{I}_{\mathfrak{W}} f\right)$ ).

Now from Lemmas 3.12 , 3.14 and 3.15 we have that $\left(h_{\mathfrak{W}},\langle\cdot, \cdot\rangle_{\mathfrak{W}}, \Gamma_{\mathfrak{W}}, V_{\mathfrak{W}}, \mathcal{T}_{\mathfrak{W}}, T_{\mathfrak{W}}, \mathfrak{I}_{\mathfrak{W}}\right)$ satisfies all conditions stated in Definition 3.3 (i) and by Theorem 3.4 (i) we get that the net of local $\mathrm{C}^{*}$-algebras above is a HK-net.

3.17 Remark (i) We will show next that the net constructed above is isomorphic to the fermionic net defined in [48, Section 3, case $n=1$ ]. Using the notation and results of the latter reference we specify the unitary $\lambda_{\mathfrak{W}}: h_{\mathfrak{W}} \rightarrow h_{1}$ (recall Proposition [3.5] (i)): for $\chi_{ \pm}, \omega_{ \pm} \in \mathrm{L}^{2}\left(\mathcal{C}_{+}, \mathbb{C}, \mu_{0}(\mathrm{~d} p)\right)$ put

$$
\begin{aligned}
\lambda_{\mathrm{F}}\left(H_{(\cdot)}\left(\begin{array}{c}
\chi_{+}(\cdot) \\
0
\end{array}\right) \oplus \overline{H_{(\cdot)}}\left(\begin{array}{c}
\chi_{-}(\cdot) \\
0
\end{array}\right) \oplus H_{(\cdot)}\left(\begin{array}{c}
\omega_{+}(\cdot) \\
0
\end{array}\right) \oplus \overline{H_{(\cdot)}}\left(\begin{array}{c}
\omega_{-}(\cdot) \\
0
\end{array}\right)\right)(p) \\
:=\left[\overline{H_{p}}\left(\begin{array}{c}
0 \\
\chi_{+}(p)
\end{array}\right)\right]_{+} \oplus\left[H_{p}\left(\begin{array}{c}
0 \\
\chi_{-}(p)
\end{array}\right)\right]_{-} \\
\oplus\left[\overline{H_{p}}\left(\begin{array}{c}
0 \\
\omega_{+}(p)
\end{array}\right)\right]_{+} \oplus\left[H_{p}\left(\begin{array}{c}
0 \\
\omega_{-}(p)
\end{array}\right)\right]_{-}
\end{aligned}
$$

where $[\cdot]_{ \pm}$denote the classes of the factor spaces $\mathfrak{H}_{ \pm}^{\prime}$ defined in [48, Section 3]. Using the statements in the proof of [48, Lemma 3.2] it is straightforward to show that $\lambda_{F}$ satisfies the properties required in Proposition 3.5 (i).

(ii) From the construction given in Remark 3.8 it can be easily shown that $P:=\left(\begin{array}{llll}\mathbb{1} & 0 & 0 & 0 \\ 0 & \mathbb{1} & 0 & 0 \\ 0 & 0 & 0 & 0 \\ 0 & 0 & 0 & 0\end{array}\right)$ is a basis projection on $h_{\mathfrak{W}}$ (i.e. $P+\Gamma_{\mathfrak{W}} P \Gamma_{\mathfrak{W}}=\mathbb{1}$ ) that characterises a Fock state on $\operatorname{CAR}\left(h_{\mathfrak{W}}, \Gamma_{\mathfrak{W}}\right)$ satisfying the spectrality condition (recall Definition 3.7 (F4)). Note that $V_{1}(a) \oplus V_{2}(a), a \in \mathbb{R}^{4}$, satisfies the spectrality condition on the one particle Hilbert space $P h_{\mathfrak{W}}=h_{+} \oplus h_{-}$.

(iii) It is also straightforward to generalise the present construction to higher (half-integer) helicity values, just replacing in the preceding construction the indeces $\left(\frac{1}{2}, 0\right)$ by $\left(\frac{n}{2}, 0\right)$ and $\left(0, \frac{1}{2}\right)$ by $\left(0, \frac{n}{2}\right)$ with $n \geq 3$ and odd. Adapting part (i) above we get the isomorphy to the corresponding nets in [48.

From the isomorphy given in (i) of the previous remark we can assume the structural results of [48, Section 5] (see also [19]). For example we have:

3.18 Corollary The net of von Neumann algebras $\mathcal{O} \mapsto \mathcal{M}_{\mathfrak{W}}(\mathcal{O})$ obtained from the Weyl net using the canonical Fock space given in Remark 3.17 (ii) transforms in addition covariantly w.r.t. the (fourthfold covering) of the conformal group. Moreover it satisfies essential duality as well as timelike duality for the forward/backward cones.

3.19 Remark The formulas for the graph of the modular operator and the modular conjugation associated to double cones given for fermionic models in [13, Theorem 5.10] can be also applied to the present construction. 


\section{$3.2 \mathfrak{F}$-net}

The construction bellow will illustrate the bosonic axioms (B1) - (B3) of Section 3 making now use of the $\mathfrak{F}$-Equation (34) (recall also the definitions and results in Subsection 2.8). As in the Weyl case the following computation of intertwiner spaces will be essential for the construction of the corresponding embedding. The proof of the following result is simmilar as in Lemma 3.11]

3.20 Lemma Recalling the notion of intertwiner space in Subsection 3.1] we have:

$$
\begin{aligned}
\left(D^{(1,0)}(\mathcal{E}(2)), D^{(0,1)}(\mathcal{E}(2))\right) & =\left\{s\left(\begin{array}{ll}
0 & 1 \\
0 & 0
\end{array}\right) \otimes\left(\begin{array}{ll}
0 & 1 \\
0 & 0
\end{array}\right) \mid s \in \mathbb{R}\right\}, \\
\left(D^{(1,0)}(\mathcal{E}(2)), D^{(1,0)}(\mathcal{E}(2))\right) & =\mathbb{C} \mathbb{1} .
\end{aligned}
$$

Next, consider the space

$$
h_{\mathfrak{F}}:=h_{+} \oplus h_{-},
$$

as a real space with nondegenerate symplectic form given by

$$
\sigma_{\mathfrak{F}}(\varphi, \psi):=\operatorname{Im}\langle\varphi, \psi\rangle_{\mathfrak{F}}=\frac{1}{2 i}\left(\langle\varphi, \psi\rangle_{\mathfrak{F}}-\langle\psi, \varphi\rangle_{\mathfrak{F}}\right),
$$

where $\langle\cdot, \cdot\rangle_{\mathfrak{F}}:=\langle\cdot, \cdot\rangle_{+} \oplus\langle\cdot, \cdot\rangle_{-}$and $\varphi, \psi \in h_{\mathfrak{F}}$. The $\mathrm{C}^{*}$-algebra $\operatorname{CCR}\left(h_{\mathfrak{F}}, \sigma_{\mathfrak{F}}\right)$ is simple and uniquely given by [51].

The reducible representation

$$
V_{\mathfrak{F}}:=V_{+} \oplus V_{-},
$$

where for $g=(A, a) \in \mathrm{SL}(2, \mathbb{C}) \ltimes \mathbb{R}^{4}, \varphi \in h_{+}$and $\psi \in h_{-}$we define

$$
\left(V_{+}(g) \varphi\right)(p):=e^{-i p a} D^{(1,0)}(A) \varphi\left(\Lambda_{A}^{-1} p\right) \text { and }\left(V_{-}(g) \psi\right)(p):=e^{-i p a} D^{(0,1)}(A) \psi\left(\Lambda_{A}^{-1} p\right),
$$

leaves the real-bilinear form $\langle\cdot, \cdot\rangle_{\mathfrak{F}}$ invariant and, therefore, the symplectic form $\sigma_{\mathfrak{F}}$ is also $V_{\mathfrak{F}^{-}}$ invariant. In the rest of the section we will also write the finite-dimensional representation $D^{(1,0)}(A)$ simply as $D(A)$ and $D^{(0,1)}(A)$ as $D(\bar{A}), A \in \mathrm{SL}(2, \mathbb{C})$.

Define also the covariant representation for the present model (which satisfies the support properties required in Definition 3.3 (i)):

$$
\left(T_{\mathfrak{F}}(g) f\right)(x):=D(\bar{A}) f\left(\Lambda_{A}^{-1}(x-a)\right), \quad g=(A, a) \in \mathrm{SL}(2, \mathbb{C}) \ltimes \mathbb{R}^{4}, f \in \mathcal{S}\left(\mathbb{R}^{4}, \mathcal{H}^{(0,1)}\right)=: \mathcal{T}_{\mathfrak{F}} .
$$

In analogy to the Weyl case we introduce the following embeddings $\mathfrak{I}_{1}: \mathcal{S}\left(\mathbb{R}^{4}, \mathcal{H}\right) \longrightarrow h_{+}$and $\mathfrak{I}_{2}: \mathcal{S}\left(\mathbb{R}^{4}, \mathcal{H}\right) \longrightarrow h_{-}$defined for all $f \in \mathcal{S}\left(\mathbb{R}^{4}, \mathcal{H}\right)$ by

$$
\begin{aligned}
& \left(\Im_{1} f\right)(p):=D\left(H_{p}\right) D\left(\left(\begin{array}{l}
01 \\
0
\end{array}\right)\right) D\left(\overline{H_{p}}\right)^{-1} \widehat{f}(p), \quad p \in \mathcal{C}_{+}^{\circ},
\end{aligned}
$$

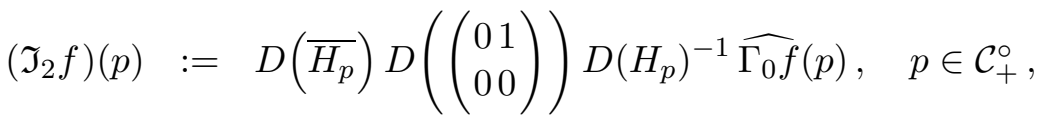

where $D\left(\left(\begin{array}{l}0 \\ 0 \\ 0\end{array}\right)\right) \in\left(D^{(1,0)}(\mathcal{E}(2)), D^{(0,1)}(\mathcal{E}(2))\right)$ and where the 'hat' ^ means the Fourier transformation which is restricted to $\mathcal{C}_{+}^{\circ}$ as in the Weyl case. Further $\Gamma_{0}: \mathcal{H}^{(0,1)} \rightarrow \mathcal{H}^{(1,0)}$ is again an anti-unitary involution (conjugation).

Finally, the embedding that specifies the net structure and satisfies the conditions stated in Subsection 2.6 is given by

$$
\mathfrak{I}_{\mathfrak{F}}: \mathcal{T}_{\mathfrak{F}} \longrightarrow h_{\mathfrak{F}}, \text { with } \mathfrak{I}_{\mathfrak{F}} f:=\mathfrak{I}_{1} f \oplus \mathfrak{I}_{2} f \text {. }
$$

The covariance property of the net characterised by the preceding embedding is guaranteed by the following result: 
3.21 Lemma Using the notation introduced above the equation $\mathfrak{I}_{\mathfrak{F}} T_{\mathfrak{F}}(g)=V_{\mathfrak{F}}(g) \mathfrak{I}_{\mathfrak{F}}$ holds for all $g \in \widetilde{\mathcal{P}_{+}^{\uparrow}}$.

Proof: The proof is done similarly as in Lemma 3.14. The intertwining equation is now based on the relations,

$$
\left.\begin{array}{rl}
\mathfrak{I}_{1} T_{\mathfrak{F}}(g) & =V_{+}(g) \mathfrak{I}_{1} \\
\mathfrak{I}_{2} \Gamma_{0} T_{\mathfrak{F}}(g) & =V_{-}(g) \mathfrak{I}_{2} \Gamma_{0},
\end{array}\right\}
$$

for any $g \in \widetilde{\mathcal{P}_{+}^{\uparrow}}$.

The next result will ensure the causality property of the net associated to embedding $\mathfrak{I}_{\mathfrak{F}}$.

3.22 Lemma Suppose that $\operatorname{supp} f \perp \operatorname{supp} k$ for $f, k \in \mathcal{T}_{\mathfrak{F}}$. Then $\sigma_{\mathfrak{F}}\left(\mathfrak{I}_{\mathfrak{F}} f, \mathfrak{I}_{\mathfrak{F}} k\right)=0$ holds.

Proof: First note that $\mathfrak{I}_{2}\left(\Gamma_{0} f\right)(p)=\Gamma_{0}\left(\mathfrak{I}_{1} f\right)(-p)$ for all $f \in \mathcal{S}\left(\mathbb{R}^{4}, \mathcal{H}\right)$. Then, computing similarly as in Lemma 3.15 (putting now $\widetilde{\beta_{+}}(p):=D\left(H_{p}^{-1}\right)^{*} D\left(H_{p}^{-1}\right)$ and $\left.\beta_{-}(p):=\Gamma_{0} \beta_{+}(p) \Gamma_{0}\right)$, we get

$$
\begin{aligned}
\sigma_{\mathfrak{F}}\left(\mathfrak{I}_{\mathfrak{F}} f, \mathfrak{I}_{\mathfrak{F}} k\right)=\sigma_{\mathfrak{F}}\left(\mathfrak{I}_{1} f \oplus \mathfrak{I}_{2}\left(\Gamma_{0} f\right), \mathfrak{I}_{1} k \oplus \mathfrak{I}_{2}\left(\Gamma_{0} k\right)\right) \\
=\frac{1}{2 i}\left(\int_{\mathcal{C}_{+}}\left\langle\widehat{f}(p), \beta_{+}(p) \widehat{k}(p)\right\rangle_{\mathbb{C}^{4}} \mu_{0}(\mathrm{~d} p)+\int_{\mathcal{C}_{+}}\left\langle\widehat{k}(-p), \beta_{+}(p) \widehat{f}(-p)\right\rangle_{\mathbb{C}^{4}} \mu_{0}(\mathrm{~d} p)\right. \\
\left.\quad-\int_{\mathcal{C}_{+}}\left\langle\widehat{k}(p), \beta_{+}(p) \widehat{f}(p)\right\rangle_{\mathbb{C}^{4}} \mu_{0}(\mathrm{~d} p)-\int_{\mathcal{C}_{+}}\left\langle\widehat{f}(-p), \beta_{+}(p) \widehat{k}(-p)\right\rangle_{\mathbb{C}^{4}} \mu_{0}(\mathrm{~d} p)\right) \\
=0,
\end{aligned}
$$

where the last equation follows from the fact that the matrix elements of the operator-valued function (recall Remark 2.13)

$$
\begin{aligned}
\beta_{+}(p) & :=D\left(\left({\overline{H_{p}^{-1}}}^{*}\left(\begin{array}{ll}
0 & 0 \\
0 & 1
\end{array}\right){\overline{H_{p}}}^{-1}\right)\right. \\
& \cong \frac{1}{4}\left(\begin{array}{crrr}
\left(p_{0}-p_{3}\right)^{2} & -\left(p_{0}-p_{3}\right)\left(p_{1}+i p_{2}\right) & -\left(p_{0}-p_{3}\right)\left(p_{1}+i p_{2}\right) & \left(p_{1}+i p_{2}\right)^{2} \\
-\left(p_{0}-p_{3}\right)\left(p_{1}+i p_{2}\right) & \left(p_{0}+p_{3}\right)\left(p_{0}-p_{3}\right) & \left(p_{0}+p_{3}\right)\left(p_{0}-p_{3}\right) & -\left(p_{0}+p_{3}\right)\left(p_{1}+i p_{2}\right) \\
-\left(p_{0}-p_{3}\right)\left(p_{1}+i p_{2}\right) & \left(p_{0}+p_{3}\right)\left(p_{0}-p_{3}\right) & \left(p_{0}+p_{3}\right)\left(p_{0}-p_{3}\right) & -\left(p_{0}+p_{3}\right)\left(p_{1}+i p_{2}\right) \\
\left(p_{1}-i p_{2}\right)^{2} & -\left(p_{0}+p_{3}\right)\left(p_{1}-i p_{2}\right) & -\left(p_{0}+p_{3}\right)\left(p_{1}-i p_{2}\right) & \left(p_{0}+p_{3}\right)^{2}
\end{array}\right)
\end{aligned}
$$

are homogeneous polynomials of degree 2 (see Lemma 3.10).

We will show the existence of a free net associated to the $\mathfrak{F}$-Equation, which we call $\mathfrak{F}$-net for short.

3.23 Theorem Consider the net of local linear submanifolds of $h_{\mathfrak{F}}$ given for $\mathcal{O} \in \mathcal{B}\left(\mathbb{R}^{4}\right)$ by

$$
\mathbb{R}^{4} \supset \mathcal{O} \longmapsto h_{\mathfrak{F}}(\mathcal{O}):=\left\{\mathfrak{I}_{\mathfrak{F}} f \mid f \in \mathrm{C}_{0}^{\infty}\left(\mathbb{R}^{4}, \mathcal{H}\right), \operatorname{supp} f \subset \mathcal{O}\right\}
$$

Then the net of local $C^{*}$-algebra defined by

$$
\mathbb{R}^{4} \supset \mathcal{O} \longmapsto \mathcal{A}_{\mathfrak{F}}(\mathcal{O}):=\mathrm{C}^{*}\left(\left\{W(\varphi) \mid \varphi \in h_{\mathfrak{F}}(\mathcal{O})\right\}\right),
$$

where the $W(\cdot)$ 's denote the generators of the $C^{*}$-algebra $\mathrm{CCR}\left(h_{\mathfrak{F}}, \sigma_{\mathfrak{F}}\right)$, is a HK-net. 
Proof: By Lemmas 3.21 and 3.22 we have that the tuple $\left(h_{\mathfrak{F}}, \sigma_{\mathfrak{F}}, V_{\mathfrak{F}}, \mathcal{T}_{\mathfrak{F}}, T_{\mathfrak{F}}, \mathfrak{I}_{\mathfrak{F}}\right)$ satisfies all conditions stated in Definition [3.3 (ii) and again by Theorem [3.4 (ii) we get that the net of local $\mathrm{C}^{*}$-algebras above is a HK-net.

3.24 Remark (i) We will show next that the net constructed above is isomorphic to the bosonic net defined in [48, Section 3, case $n=2$ ]. Using the notation and results of the latter reference we specify the symplectic bijection $\lambda_{\mathrm{B}}: h_{\mathfrak{F}} \rightarrow h_{2}$ (recall Proposition [3.5 (ii)): for $\chi_{ \pm} \in \mathrm{L}^{2}\left(\mathcal{C}_{+}, \mathbb{C}, \mu_{0}(\mathrm{~d} p)\right)$ put

$$
\begin{aligned}
\lambda_{\mathrm{B}}\left(D\left(H_{(\cdot)}\right)\left(\begin{array}{l}
1 \\
0
\end{array}\right) \otimes\left(\begin{array}{l}
1 \\
0
\end{array}\right) \chi_{+}(\cdot) \oplus D\left(\overline{H_{(\cdot)}}\right)\left(\begin{array}{l}
1 \\
0
\end{array}\right) \otimes\left(\begin{array}{l}
1 \\
0
\end{array}\right) \chi_{-}(\cdot)\right)(p) \\
:=\left[D\left(H_{p}\right)\left(\begin{array}{l}
0 \\
1
\end{array}\right) \otimes\left(\begin{array}{l}
0 \\
1
\end{array}\right) \chi_{+}(p)\right]_{+} \oplus\left[D\left(H_{p}\right)\left(\begin{array}{l}
0 \\
1
\end{array}\right) \otimes\left(\begin{array}{l}
0 \\
1
\end{array}\right) \chi_{-}(p)\right]_{-},
\end{aligned}
$$

where $[\cdot]_{ \pm}$denote the clases of the factor spaces $\mathfrak{H}_{ \pm}^{\prime}$ defined in [48, Section 3]. Using again the statements in the proof of [48. Lemma 3.2] it is straightforward to prove that $\lambda_{\mathrm{F}}$ satisfies the properties required in Proposition 3.5 (ii). (An isometry to the free net constructed in [12, Part B] is given in [47, Remark 3.3.5].)

(ii) The natural complexification of $h_{\mathfrak{F}}$ given by $J\left(\varphi_{+} \oplus \varphi_{-}\right):=i \varphi_{+} \oplus i \varphi_{-}, \varphi_{+} \oplus \varphi_{-} \in h_{\mathfrak{F}}$, already defines a Fock state satisfying the spectrum condition (cf. Definition 3.7 (B3) and 14. Subsection 8.2.3]) and where the one-particle Hilbert space carries the representation usually considered in the literature for describing photons with both helicities [40, Section 2].

(iii) It is now obvious that as in the Fermi case we may generalise the preceding construction to arbitrary values of the integer helicity parameter $n \in \mathbb{N}$. Replace the index $(1,0)$ by $(n, 0)$ and $(0,1)$ by $(0, n)$ etc. Thus we have produced (considering Remark 3.17(i)) isomorphic nets to the ones given in [48. Note also that the use of the direct sum of 4 reference spaces in the Fermi case was forced by the self-dual approach to the CAR-algebra. Nevertheless in the Bose and Fermi cases the corresponding one-particle Hilbert spaces given by the canonical Fock states (cf. Remark 3.17 (ii)) are of the form $h_{+} \oplus h_{-}$.

3.25 Remark From Theorem 2.31 we can show the equivalence of the $\mathrm{C}^{*}$-dynamical systems $\left(\operatorname{CCR}\left(h_{\mathfrak{F}}, \sigma_{\mathfrak{F}}\right), \alpha_{g}^{\mathfrak{F}}, \widetilde{\mathcal{P}_{+}^{\uparrow}}\right)$ and $\left(\operatorname{CCR}\left(h_{\mathfrak{A}}, \sigma_{\mathfrak{A}}\right), \alpha_{g}^{\mathfrak{A}}, \widetilde{\mathcal{P}_{+}^{\uparrow}}\right)$, where $\sigma_{\mathfrak{A}}:=\operatorname{Im}\langle\cdot, \cdot\rangle_{\mathfrak{A}}$ and $\alpha_{g}^{\mathfrak{A}}$ is the Bogoljubov automorphism associated to $V_{\mathfrak{A}}$ (recall Subsection 2.9). But due to the specific form of the factor space $h_{\mathfrak{A}}$ and the corresponding covariant representation $T_{\mathfrak{A}}$ there does not exist a nontrivial embedding $\mathfrak{I}_{\mathfrak{A}}$ satisfying the corresponding intertwining property with $V_{\mathfrak{A}}$ (cf. axiom (B2)). The impossibility of constructing the free net associated to the vector potential is the analogue in our context of the well-known Strocchi no-go theorems, that are formulated in the quantum field theoretical context (cf. 67]). For a detailed treatment of the nets associated to the electromagnetic vector potential (including a general analysis of the localised constraints) see 32 .

\section{Massless quantum fields}

In the previous section we have seen that the embeddings that characterise the massless free nets naturally reduce the degrees of freedom in the fibre (cf. Remark 2.11]and Eqs. (60), (62)) by using elements $\left(\begin{array}{l}0 \\ 0\end{array}\right)$ of intertwiner space between the little group $\mathcal{E}(2)$ and its conjugate $\overline{\mathcal{E}(2)}$. This choice shows explicitly that the embeddings map the test functions into the space of solutions of massless relativistic wave equations. Now using the canonical Fock states associated to the CAR- 
and CCR-algebras (recall Remarks 3.17 and 3.24 (ii)) we will obtain in a natural way quantum fields that satisfy in the distributional sense the Weyl and Maxwell equations. Since these two cases are typical (see Remarks [2.26 (ii), 3.17] and 3.24) the following procedure establishes a neat way to define massless fields for any helicity value. This construction is considerably simpler than what is done usually in QFT, where so-called $2 j+1$ quantum fields are introduced (a clear reminiscence of the massive case) and then constrained by imposing suitable equations on them [74, 40, Section 2].

If one considers the canonical Fock states mentioned before, then one can also interpret the embeddings $\mathfrak{I}$, that where used to completely characterise the free nets in Theorems 3.16 and 3.23 as a one particle Hilbert structure. Indeed, $\mathfrak{I}$ can be seen as a real linear map from $\mathcal{T}:=\mathcal{S}\left(\mathbb{R}^{4}, \mathcal{H}^{\left(0, \frac{n}{2}\right)}\right)$ into the (complex) one-particle Hilbert space

$$
\mathfrak{H}_{1} \subset h_{+} \oplus h_{-}
$$

(with scalar product $\langle\cdot, \cdot\rangle:=\langle\cdot, \cdot\rangle_{+} \oplus\langle\cdot, \cdot\rangle_{-}$). By Propositions 2.21, 2.24 and Remark 2.26 (ii) the one particle Hilbert space $\mathfrak{H}_{1}$ carries representations of the Poincaré group equivalent to the massless, positive energy, Wigner representations with helicities $\pm \frac{n}{2}$. Thus we can use $\mathfrak{I}$ to construct canonically massless free quantum fields. We will treat the Weyl (fermionic) and the Maxwell (bosonic) case separately. The fermionic/bosonic fields are defined on the antisymmetric/symmetric Fock space $\mathcal{F}_{a}\left(\mathfrak{H}_{1}\right) / \mathcal{F}_{s}\left(\mathfrak{H}_{1}\right)$ over the corresponding one-particle Hilbert spaces $\mathfrak{H}_{1}$. We denote the corresponding vacua simply by $\Omega$ and the scalar products by $\langle\cdot, \cdot\rangle_{a / s}$.

Free Weyl quantum field: Consider the $\mathrm{C}^{*}$-algebra $\mathrm{CAR}\left(h_{\mathfrak{W}}, \Gamma_{\mathfrak{W}}\right)$ defined in Section 3.1 and the basis projection $P:=\left(\begin{array}{llll}\mathbb{1} & 0 & 0 & 0 \\ 0 & \mathbb{1} & 0 & 0 \\ 0 & 0 & 0 & 0 \\ 0 & 0 & 0 & 0\end{array}\right)$ specified in Remark 3.17 (ii) (see also Remark 3.8 (i)). Recall that in this context the creation and annihilation operators are given as follows: for $\psi, \psi_{1}, \ldots, \psi_{n} \in \mathfrak{H}_{1}:=P\left(\overline{\mathfrak{I}_{\mathfrak{W}}\left(\mathcal{T}_{\mathfrak{W}}\right)}\right) \subset h_{+} \oplus h_{-}$we put

$$
\begin{aligned}
c(\psi) \Omega & :=0 \\
c(\psi)\left(\psi_{1} \wedge \ldots \wedge \psi_{n}\right) & :=\sqrt{n} \sum_{l=1}^{n}(-1)^{l-1}\left\langle\psi, \psi_{l}\right\rangle \psi_{1} \wedge \ldots \hat{\psi}_{l} \ldots \wedge \psi_{n} \\
c(\psi)^{*} \Omega & =\psi \\
c(\psi)^{*}\left(\psi_{1} \wedge \ldots \wedge \psi_{n}\right) & =\frac{1}{\sqrt{n+1}} \psi \wedge \psi_{1} \wedge \ldots \wedge \psi_{n},
\end{aligned}
$$

where the wedges mean the antisymmetrised tensor product

$$
\psi_{1} \wedge \ldots \wedge \psi_{n}:=\sum_{\sigma \in \mathbb{P}_{n}} \operatorname{sgn}(\sigma) \psi_{\sigma\left(a_{1}\right)} \otimes \ldots \otimes \psi_{\sigma\left(a_{n}\right)} .
$$

The previous creation and annihilation operators are mutually adjoint w.r.t. $\langle\cdot, \cdot\rangle_{a}$ and satisfy the usual anticommutation relations: for $\psi, \psi^{\prime} \in \mathfrak{H}_{1}$ one has

$$
\left[c(\psi), c\left(\psi^{\prime}\right)^{*}\right]_{+}=\left\langle\psi, \psi^{\prime}\right\rangle_{a} \mathbb{1}
$$

where $[\cdot, \cdot]_{+}$denotes the anticommutator.

In this context we may define the free Weyl quantum field as follows (recall that $\Gamma_{\mathfrak{W}} \mathfrak{I}_{\mathfrak{W}}=\mathfrak{I}_{\mathfrak{W}}$ ): 
4.1 Definition Let $\omega_{P}$ be the Fock state corresponding to the basis projection $P$ and denote by $\left(\mathcal{F}_{a}\left(\mathfrak{H}_{1}\right), \Pi_{P}, \Omega\right)$ the corresponding GNS-data. We define the free Weyl quantum field acting on $\mathcal{F}_{a}\left(\mathfrak{H}_{1}\right)$ by

$$
\phi_{\mathfrak{W}}(f):=\frac{1}{\sqrt{2}}\left(\Pi_{P}\left(\mathfrak{a}\left(\mathfrak{I}_{\mathfrak{W}} f\right)\right)\right)=\frac{1}{\sqrt{2}}\left(c\left(P\left(\mathfrak{I}_{\mathfrak{W}} f\right)\right)^{*}+c\left(P\left(\mathfrak{I}_{\mathfrak{W}} f\right)\right)\right),
$$

where $f \in \mathcal{T}_{\mathfrak{W}}:=\mathcal{S}\left(\mathbb{R}^{4}, \mathcal{H}^{\left(0, \frac{1}{2}\right)}\right)$ and $\mathfrak{a}(\cdot)$ denote the (abstract) generators of $\operatorname{CAR}\left(h_{\mathfrak{W}}, \Gamma_{\mathfrak{W}}\right)$.

4.2 Theorem The embedding $\mathfrak{I}_{\mathfrak{W}}(c f . E q .(60)$ ) is continuous w.r.t. the corresponding Schwartz and Hilbert space topologies.

Proof: It is enough to show the continuity of $\mathfrak{I}_{1}$. Recall that for $p \in \mathcal{C}_{+}$the scalar product is characterised by the positive matrix-valued function $\beta_{+}(p)=\frac{1}{2}\left(\begin{array}{cc}p_{0}-p_{3} & -p_{1}+i p_{2} \\ -p_{1}-i p_{2} & p_{0}+p_{3}\end{array}\right)$. Then we have the estimates

$$
\begin{aligned}
\left\|\mathfrak{I}_{1} f\right\|_{+}^{2} & =\int_{\mathcal{C}_{+}}\left\langle\widehat{f}(p), \beta_{+}(p) \widehat{f}(p)\right\rangle_{\mathbb{C}^{2}} \mu_{0}(\mathrm{~d} p) \\
& \leq \sum_{C^{\prime}, C=0}^{1} \int_{\mathcal{C}_{+}}\left|\beta_{+, C^{\prime} C}(p)\right| \cdot\left|\widehat{f^{C^{\prime}}}(p)\right| \cdot\left|\widehat{f^{C}}(p)\right| \mu_{0}(\mathrm{~d} p) \\
& \leq \sum_{C^{\prime}, C=0}^{1} \int_{\mathbb{R}^{3} \backslash\{0\}}|\mathbf{p}| \cdot\left|\widehat{f^{C^{\prime}}}(|\mathbf{p}|, \mathbf{p})\right| \cdot\left|\widehat{f^{C}}(|\mathbf{p}|, \mathbf{p})\right| \frac{\mathrm{d}^{3} \mathbf{p}}{|\mathbf{p}|} \\
& =\sum_{C^{\prime}, C=0}^{1} \int_{\mathbb{R}^{3} \backslash\{0\}}\left|\widehat{f^{C^{\prime}}}(|\mathbf{p}|, \mathbf{p})\right| \cdot|\widehat{f C}(|\mathbf{p}|, \mathbf{p})| \cdot \frac{\left(1+|\mathbf{p}|^{2}\right)^{4}}{\left(1+|\mathbf{p}|^{2}\right)^{4}} \mathrm{~d}^{3} \mathbf{p} \\
& \leq M\left(\sum_{C^{\prime}, C=0}^{1}\left\|\widehat{f^{C^{\prime}}}\right\|_{4,0} \cdot\left\|\widehat{f^{C}}\right\|_{4,0}\right),
\end{aligned}
$$

where $M=\int_{\mathbb{R}^{3}} \frac{1}{\left(1+|\mathbf{p}|^{2}\right)^{4}} \mathrm{~d}^{3} \mathbf{p}$ and $\left\|\widehat{f^{C}}\right\|_{4,0}:=\sup _{p \in \mathbb{R}^{4}}\left\{\left(1+|p|^{2}\right)^{2}\left|\widehat{f^{C}}(p)\right|\right\}$ is a particular seminorm corresponding to the Schwartz space topology. Suppose now that $f_{n} \rightarrow 0$ in the topology of $\mathcal{T}_{\mathfrak{W}}:=\mathcal{S}\left(\mathbb{R}^{4}, \mathcal{H}^{\left(0, \frac{1}{2}\right)}\right)$. Then by the continuity of Fourier transformation and the previous estimates we conclude that $\mathfrak{I}_{1}\left(f_{n}\right) \rightarrow 0$ in $h_{+}$and the proof is concluded.

In the following theorem we will show that the quantum field defined previously satisfies the Wightman axioms as well as the Weyl equation in the distributional sense.

4.3 Theorem The Weyl quantum field $\phi_{\mathfrak{W}}(f), f \in \mathcal{T}_{\mathfrak{W}}$, defined on $\mathcal{F}_{a}\left(\mathfrak{H}_{1}\right)$ is a bounded, selfadjoint operator. Moreover we have

(i) (Weyl equation) $\phi_{\mathfrak{W}}(\cdot)$ satisfies the Weyl equation in the distributional sense:

$$
\phi_{\mathfrak{W}}\left(\partial^{C C^{\prime}} f_{C}\right)=0, \quad f \in \mathcal{S}\left(\mathbb{R}^{4}, \mathcal{H}^{\left(\frac{1}{2}, 0\right)}\right) .
$$

(ii) (Poincaré invariance and spectral condition) $\phi_{\mathfrak{W}}(\cdot)$ transforms covariantly under the Poincaré group: Let $T_{\mathfrak{W}}$ and $Q$ be the covariant and the second quantisation of the canonical representation $P V_{\mathfrak{W}}=V_{1} \oplus V_{2}$ on $\mathcal{F}_{a}\left(\mathfrak{H}_{1}\right)$, respectively (recall Section [3.1). Then

$$
Q(g) \phi_{\mathfrak{W}}(f) Q(g)^{-1}=\phi_{\mathfrak{W}}\left(T_{\mathfrak{W}} f\right), \quad f \in \mathcal{T}_{\mathfrak{W}}, \quad g \in \widetilde{\mathcal{P}_{+}^{\uparrow}} .
$$

Further, the representation $V_{1} \oplus V_{2}$ satisfies the spectral condition on $\mathfrak{H}_{1}$ and $Q(g) \Omega=\Omega$, $g \in \widetilde{\mathcal{P}_{+}^{\uparrow}}$. 
(iii) (Anticommutation relations) For $f, k \in \mathcal{T}_{\mathfrak{W}}$ such that $\operatorname{supp} f$ and $\operatorname{supp} k$ are space-like separated, the anticommutator of the corresponding smeard fields vanishes:

$$
\left[\phi_{\mathfrak{W}}(f), \phi_{\mathfrak{W}}(k)\right]_{+}=0 .
$$

(iv) (Regularity) The map

$$
\mathcal{T}_{\mathfrak{W}} \ni f \mapsto\left\langle\psi_{1}, \phi_{\mathfrak{W}}(f) \psi_{2}\right\rangle_{a}, \quad \psi_{1}, \psi_{2} \in \mathcal{F}_{a}\left(\mathfrak{H}_{1}\right),
$$

is a tempered distribution.

Proof: The boundedness and self-adjointness of the field follows from the same properties of the generator $\mathfrak{a}\left(\mathfrak{I}_{\mathfrak{w}} f\right)$ of the CAR-algebra.

To show (i) recall that e.g. the embedding $\mathfrak{I}_{1}$ used to specify $\mathfrak{I}_{\mathfrak{W}}$ maps into the space of solutions of Weyl equation (cf. ([60) $)$. Indeed, we will show that $\mathfrak{I}_{1}\left(\partial^{C C^{\prime}} f_{C}\right)=0, f \in \mathcal{S}\left(\mathbb{R}^{4}, \mathcal{H}^{\left(\frac{1}{2}, 0\right)}\right)$ : for $B \in\{0,1\}$ and summing over repeated indices we have

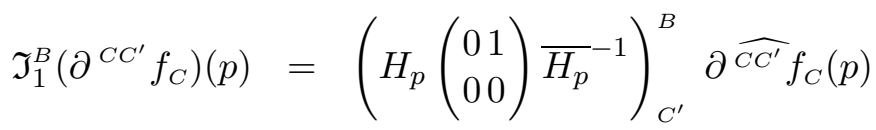

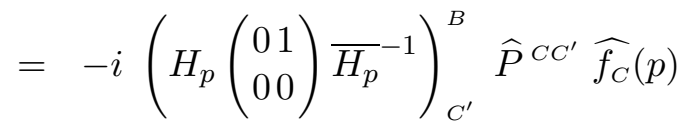

$$
\begin{aligned}
& =0 \text {, }
\end{aligned}
$$

where the last equation follows from the fact that

$$
\left(\widehat{P}^{C C^{\prime}}\right)=\left(\begin{array}{cc}
p_{0}+p_{3} & p_{1}+i p_{2} \\
p_{1}-i p_{2} & p_{0}-p_{3}
\end{array}\right)=\overline{H_{p}}\left(\begin{array}{l}
20 \\
00
\end{array}\right){\overline{H_{p}}}^{*} .
$$

Similarly we obtain $\mathfrak{I}_{2}\left(\partial^{C C^{\prime}} f_{C}\right)=0$, hence $P\left(\mathfrak{I}_{\mathfrak{W}}\left(\partial^{C C^{\prime}} f_{C}\right)\right)=0$ and the field satisfies the Weyl equation as required:

$$
\phi_{\mathfrak{W}}\left(\partial^{C C^{\prime}} f_{C}\right)=c\left(P\left(\mathfrak{I}_{\mathfrak{W}}\left(\partial^{C C^{\prime}} f_{C}\right)\right)\right)^{*}+c\left(P\left(\mathfrak{I}_{\mathfrak{W}}\left(\partial^{C C^{\prime}} f_{C}\right)\right)\right)=0 .
$$

The property (ii) follows from Remark 3.8 (i). The anticommutation of the field in (iii) is a consequence of the anticommutation of the generators $\mathfrak{a}\left(\mathfrak{I}_{\mathfrak{W}} f\right)$ and $\mathfrak{a}\left(\mathfrak{I}_{\mathfrak{W}} k\right)$ of the CAR-algebra (cf. Lemma 3.15 and Theorem 3.16).

The regularity (iv) of the field follows from the continuity of the embedding $\mathfrak{I}_{\mathfrak{W}}$ (see Theorem 4.2). Indeed, let $f_{n} \rightarrow 0$ in the Schwartz topology of $\mathcal{T}_{\mathfrak{2}}$. Then by Theorem 4.2 we have $\mathfrak{I}_{\mathfrak{W}} f_{n} \rightarrow 0$ in the Hilbert space topology. Now for any $\psi \in \mathcal{F}_{a}\left(\mathfrak{H}_{1}\right)$

$$
\left\|\phi_{\mathfrak{W}}\left(f_{n}\right) \psi\right\| \leq\left\|\mathfrak{a}\left(\mathfrak{I}_{\mathfrak{W}} f_{n}\right)\right\|_{\mathrm{C}^{*}}\|\psi\| \leq\left\|\mathfrak{I}_{\mathfrak{W}} f_{n}\right\|\|\psi\|
$$

and therefore $\mathrm{s}-\lim _{n \rightarrow \infty} \phi_{\mathfrak{W}}\left(f_{n}\right)=0$. The strong continuity of the field implies finally the temperedness of the distribution.

We show in the next theorem some additional properties satisfied by the Weyl quantum field.

4.4 Theorem The field $\phi_{\mathfrak{W}}$ transforms in addition covariantly w.r.t. the (fourfold cover of) conformal group in Minkowski space $\mathrm{SU}(2,2)$.

Proof: The extension of the covariance property to the conformal group follows from the results in [48, Section 5] (recall also the isomorphy between the massless free nets of Section 3.1 and those constructed in 48] given Remark 3.17 (i)). 
4.5 Remark As mentioned above, the Weyl case is typical for fermionic models with nontrivial (half-integer) helicity. Hence replacing for example $\left(0, \frac{1}{2}\right)$ by $\left(0, \frac{n}{2}\right)$ with $n \geq 3$ and odd, one can similarly define the massless fermionic free quantum field with helicity $\frac{n}{2}$ by

$$
\phi_{n}(f):=\frac{1}{\sqrt{2}}\left(\Pi_{P}\left(\mathfrak{a}\left(\mathfrak{I}_{n} f\right)\right)\right)=\frac{1}{\sqrt{2}}\left(c\left(P\left(\mathfrak{I}_{\mathfrak{W}} f\right)\right)^{*}+c\left(P\left(\mathfrak{I}_{\mathfrak{W}} f\right)\right)\right), \quad f \in \mathcal{T}_{n}:=\mathcal{S}\left(\mathbb{R}^{4}, \mathcal{H}^{\left(0, \frac{n}{0}\right)}\right) .
$$

These fields also satisfy Wightman axioms and the adapted version of Theorem 4.4. In particular, it satisfies the corresponding massless relativistic wave equation in the weak sense:

$$
\phi_{n}\left(\partial^{C C^{\prime}} f_{C}{ }^{\prime} \ldots C_{n-1}^{\prime}\right)=0
$$

(cf. Corollary 2.25).

Free Maxwell quantum field: Consider the simple $\mathrm{C}^{*}$-algebra $\operatorname{CCR}\left(h_{\mathfrak{F}}, \sigma_{\mathfrak{F}}\right)$ given in Section 3.2 as well as the canonical Fock state $\omega_{J}$ on $\operatorname{CCR}\left(h_{\mathfrak{F}}, \sigma_{\mathfrak{F}}\right)$ specified by the internal complexification $J\left(\varphi_{+} \oplus \varphi_{-}\right):=i \varphi_{+} \oplus i \varphi_{-}, \varphi_{+} \oplus \varphi_{-} \in h_{\mathfrak{F}}$ (recall Remarks 3.8 and 3.24 (ii)). Putting $\mathfrak{H}_{1}:=\overline{\mathfrak{I}_{\mathfrak{F}}\left(\mathcal{T}_{\mathfrak{F}}\right)} \subset h_{+} \oplus h_{-}$the generating functional is given by

$$
\mathfrak{H}_{1} \ni \psi \mapsto e^{-\frac{1}{4}\|\psi\|^{2}} .
$$

4.6 Definition Let $\omega_{J}$ be the Fock state associated to the internal complexification $J$ given above and denote by $\left(\mathcal{F}_{s}\left(\mathfrak{H}_{1}\right), \Pi_{J}, \Omega\right)$ the corresponding GNS-data. $\overline{\Phi_{J}\left(\mathfrak{I}_{\mathfrak{F}} f\right)}, f \in \mathcal{T}_{\mathfrak{F}}:=\mathcal{S}\left(\mathbb{R}^{4}, \mathcal{H}^{(0,1)}\right)$, is the infinitesimal generator of the strongly continuous unitary group

$$
\mathbb{R} \ni t \mapsto \Pi_{J}\left(W\left(t \mathfrak{I}_{\mathfrak{F}} f\right)\right)=e^{-i t \overline{\Phi_{J}\left(\mathfrak{I}_{\mathfrak{F}}^{f)}\right.}},
$$

where $W(\cdot)$ are the (abstract) generators of $\operatorname{CCR}\left(h_{\mathfrak{F}}, \sigma_{\mathfrak{F}}\right)$. Then we define the free Maxwell quantum field acting on $\mathcal{F}_{s}\left(\mathfrak{H}_{1}\right)$ by

$$
\phi_{\mathfrak{F}}(f):=\overline{\Phi_{J}\left(\mathfrak{I}_{\mathfrak{F}} f\right)}, \quad f \in \mathcal{T}_{\mathfrak{F}} .
$$

Notice that the free Maxwell quantum field is, as a consequence of the uniqueness of the GNS representation and Nelson's analytic vector theorem, the closure of the essentially self-adjoint operator

$$
\Phi_{J}\left(\mathfrak{I}_{\mathfrak{F}} f\right)=\frac{1}{\sqrt{2}}\left(a\left(\left(\mathfrak{I}_{\mathfrak{F}} f\right)\right)^{*}+a\left(\left(\mathfrak{I}_{\mathfrak{F}} f\right)\right)\right),
$$

on the set $\mathcal{F}_{\text {fin }} \subset \mathcal{F}_{s}\left(\mathfrak{H}_{1}\right)$ of finite particle vectors (cf. [58, Theorem X.41]). The creation and annihilation operators on the symmetric Fock space over the one-particle Hilbert space $\mathfrak{H}_{1}$ are defined as usual: For $\varphi, \varphi_{1}, \ldots, \varphi_{n} \in \mathfrak{H}_{1}$ we put

$$
\begin{aligned}
a(\varphi) \Omega & :=0 \\
a(\varphi) \mathcal{S}_{n}\left(\varphi_{1} \otimes \ldots \otimes \varphi_{n}\right) & :=\sqrt{n} \sum_{l=1}^{n}\left\langle\varphi, \varphi_{l}\right\rangle \mathcal{S}_{n-1}\left(\varphi_{1} \otimes \ldots \hat{\varphi}_{l} \ldots \otimes \varphi_{n}\right) \\
a(\varphi)^{*} \Omega & =\varphi \\
a(\varphi)^{*} \mathcal{S}_{n}\left(\varphi_{1} \otimes \ldots \otimes \varphi_{n}\right) & =\frac{1}{\sqrt{n+1}} \mathcal{S}_{n+1}\left(\varphi \otimes \varphi_{1} \otimes \ldots \otimes \varphi_{n}\right),
\end{aligned}
$$

where the hat means omission and $\mathcal{S}_{n}$ is the symmetrisation operator $\mathcal{S}_{n}\left(\varphi_{1} \otimes \ldots \otimes \varphi_{n}\right):=$ $\sum_{\sigma \in \mathbb{P}_{n}} \varphi_{\sigma(1)} \otimes \ldots \otimes \varphi_{\sigma(n)}$ on the $n$-tensor product space over $h_{+} \oplus h_{-}$. The previous creation 
and annihilation operators are mutually adjoint w.r.t. $\langle\cdot, \cdot\rangle_{s}$ and satisfy the usual commutation relations: for $\varphi, \varphi^{\prime} \in \mathfrak{H}_{1}$ one has

$$
\left[a(\varphi), a\left(\varphi^{\prime}\right)^{*}\right]=\left\langle\varphi, \varphi^{\prime}\right\rangle_{s} \mathbb{1}
$$

where $[\cdot, \cdot]$ denotes the commutator.

Similarly as in Theorem 4.2 we can show the following continuity statement for the embedding:

4.7 Theorem The embedding $\mathfrak{I}_{\mathfrak{F}}($ recall Eq. (62) ) is continuous w.r.t. the corresponding Schwartz and Hilbert space topologies.

We will show next that the Maxwell quantum field also satisfies the Wightman axioms as well as the Maxwell equation in a distributional sense.

4.8 Theorem The Maxwell quantum field $\phi_{\mathfrak{F}}(f), f \in \mathcal{T}_{\mathfrak{F}}$, defined on $\mathcal{F}_{s}\left(\mathfrak{H}_{1}\right)$ is an unbounded, self-adjoint operator that leaves the dense subspace $\mathcal{F}_{\text {fin }}$ invariant. Moreover we have

(i) (Maxwell equation) $\phi_{\mathfrak{F}}(\cdot)$ satisfies the following equation in the distributional sense:

$$
\phi_{\mathfrak{F}}\left(\partial^{C C^{\prime}} f_{C}^{B^{\prime}}\right)=0, \quad f \in \mathcal{S}\left(\mathbb{R}^{4}, \mathcal{H}^{\left(\frac{1}{2}, \frac{1}{2}\right)}\right) .
$$

(ii) (Poincaré invariance and spectral condition) $\phi_{\mathfrak{F}}(\cdot)$ transforms covariantly under the Poincaré group: Let $T_{\mathfrak{F}}$ and $Q$ be the covariant and the second quantisation of the canonical representation $V_{\mathfrak{F}}:=V_{1} \oplus V_{2}$ on $\mathcal{F}_{s}\left(\mathfrak{H}_{1}\right)$, respectively (recall Section [3.2). Then

$$
Q(g) \phi_{\mathfrak{F}}(f) Q(g)^{-1}=\phi_{\mathfrak{F}}\left(T_{\mathfrak{F}} f\right), \quad f \in \mathcal{T}_{\mathfrak{F}}, g \in \widetilde{\mathcal{P}_{+}^{\uparrow}} .
$$

Further, the representation $V_{1} \oplus V_{2}$ satisfies the spectral condition on $\mathfrak{H}_{1}$ and $Q(g) \Omega=\Omega$, $g \in \widetilde{\mathcal{P}_{+}^{\uparrow}}$.

(iii) (Causality) For $f, k \in \mathcal{T}_{\mathfrak{F}}$ such that $\operatorname{supp} f$ and $\operatorname{supp} k$ are space-like separated, the commutator of the corresponding smeard fields vanishes:

$$
\left[\phi_{\mathfrak{F}}(f), \phi_{\mathfrak{F}}(k)\right]=0 \quad\left(\text { on } \mathcal{F}_{\text {fin }}\right) .
$$

(iv) (Regularity) The map

$$
\mathcal{T}_{\mathfrak{F}} \ni f \mapsto\left\langle\psi_{1}, \phi_{\mathfrak{F}}(f) \psi_{2}\right\rangle_{s}, \quad \psi_{1}, \psi_{2} \in \mathcal{F}_{\text {fin }},
$$

is a tempered distribution.

Proof: The self-adjointness of the field follows from its definition as generator of a strongly continuous unitary group and the invariance of $\mathcal{F}_{\text {fin }}$ is a consequence of the remarks after Definition 4.6.

To show (i) recall that e.g. the embeddings $\mathfrak{I}_{1 / 2}$ used to specify $\mathfrak{I}_{\mathfrak{F}}$ map into the space of solutions of Maxwell equation (cf. (60) ). Reasoning as in the proof of Theorem 4.3 on obtains $\mathfrak{I}_{\mathfrak{F}}\left(\partial^{C C^{\prime}} f_{C}^{B^{\prime}}\right)=0$ and again this implies

$$
\phi\left(\partial^{C C^{\prime}} f_{C}^{B^{\prime}}\right)=\frac{1}{\sqrt{2}}\left(a\left(\left(\mathfrak{I}_{\mathfrak{F}}\left(\partial^{C C^{\prime}} f_{C}^{B^{\prime}}\right)\right)\right)^{*}+a\left(\left(\mathfrak{I}_{\mathfrak{F}}\left(\partial^{C C^{\prime}} f_{C}^{B^{\prime}}\right)\right)\right)\right)=0
$$

where $f \in \mathcal{S}\left(\mathbb{R}^{4}, \mathcal{H}^{\left(\frac{1}{2}, \frac{1}{2}\right)}\right)$. 
To prove property (ii) note that the Fock state $\omega_{J}$ is invariant w.r.t. the Bogoljubov automorphism $\alpha_{g}$ generated by $V_{\mathfrak{F}}(g)$, i.e. $\omega_{J} \circ \alpha_{g}=\omega_{J}, g \in \widetilde{\mathcal{P}_{+}^{\uparrow}}$, hence by Remark 3.8 (ii) we have $Q(g) \Omega=\Omega$. Further, $Q(g)$ also leaves $\mathcal{F}_{\text {fin }}$ invariant and for $\psi \in \mathcal{F}_{\text {fin }}$ we have

$$
Q(g) \Phi_{J}\left(\mathfrak{I}_{\mathfrak{F}} f\right) Q(g)^{-1} \psi=\Phi_{J}\left(\mathfrak{I}_{\mathfrak{F}} T_{\mathfrak{F}} f\right) \psi, \quad g \in \widetilde{\mathcal{P}_{+}^{\uparrow}} .
$$

Since both sides of the previous equation are essentially self-adjoint operators we finally obtain the covariance relation:

$$
Q(g) \phi_{\mathfrak{F}}(f) Q(g)^{-1}=\phi_{\mathfrak{F}}\left(T_{\mathfrak{F}} f\right), \quad f \in \mathcal{T}_{\mathfrak{F}} .
$$

The commutation of the field in (iii) is again a consequence of the commutation of the generators $W\left(\mathfrak{I}_{\mathfrak{F}} f\right)$ and $W\left(\mathfrak{I}_{\mathfrak{F}} k\right)$ of the CCR-algebra (cf. Lemma 3.22 and Theorem [3.23).

The regularity (iv) of the field follows from the continuity of the embedding $\mathfrak{I}_{\mathfrak{F}}$ (see Theorem 4.2). Indeed, let $f_{n} \rightarrow 0$ in the Schwartz topology of $\mathcal{T}_{\mathfrak{F}}$. Then by Theorem 4.2 we have $\mathfrak{I}_{\mathfrak{F}} f_{n} \rightarrow 0$ in the Hilbert space topology. Now for any $k$-th particle vector $\psi \in \mathcal{F}_{\text {fin }}$ we have

$$
\left\|\phi_{\mathfrak{F}}\left(f_{n}\right) \psi\right\| \leq \sqrt{2} \sqrt{k+1}\left\|\mathfrak{I}_{\mathfrak{F}} f_{n}\right\|\|\psi\|
$$

and therefore $\phi_{\mathfrak{F}}\left(f_{n}\right) \rightarrow 0$ strongly on $\mathcal{F}_{\text {fin }}$. The strong continuity of the field implies finally the temperedness of the distribution.

We show in the next theorem some additional properties satisfied by the Maxwell quantum field.

4.9 Theorem The field $\phi_{\mathfrak{F}}$ transforms in addition covariantly w.r.t. the (fourfold cover of) conformal group in Minkowski space $\mathrm{SU}(2,2)$.

Proof: The extension of the covariance property to the conformal group follows from the results in [48, Section 5] (recall also the isomorphy between the massless free nets of Section 3.1 and those constructed in [4] given Remark 3.17(i)).

4.10 Remark The present construction can also be generalised to produce massless bosonic free fields with nontrivial (integer) helicity. Hence replacing for example $(0,1)$ by $(0, n)$ with $n \geq 2$ and even, one can similarly define the massless fermionic free quantum field with helicity $\frac{n}{2}$ by

$$
\phi_{n}(f):=\frac{1}{\sqrt{2}}\left(a\left(\left(\mathfrak{I}_{n} f\right)\right)^{*}+a\left(\left(\mathfrak{I}_{n} f\right)\right)\right), \quad f \in \mathcal{T}_{n}:=\mathcal{S}\left(\mathbb{R}^{4}, \mathcal{H}^{\left(0, \frac{n}{2}\right)}\right) .
$$

These fields also satisfy Wightman axioms and the adapted version of Theorem4.9. In particular, it satisfies the corresponding massless relativistic wave equation in the weak sense:

$$
\phi_{n}\left(\partial^{C C^{\prime}} f_{C}{ }^{\prime \prime} \ldots C_{n-1}^{\prime}\right)=0
$$

(cf. Corollary 2.25).

\section{Conclusions}

In a recent paper Brunetti, Guido and Longo proposed a construction procedure for a bosonic net of von Neumann algebras canonically associated to a positive energy strongly continuous (anti-) unitary Hilbert space representation of the proper Poincaré group $\mathcal{P}_{+}$(cf. [20]). They also used the suggestive name of free net as in [14, Example 8.3.1] (see also [12, 48]), since the construction 
avoids the use of quantum fields as 'coordinates' of the corresponding net. The construction of bosonic free nets in Section 3 and the one in [20] are similar in that both use Wigner's cornerstone analysis of the unitary irreducible representations of the universal cover of the Poincare group, as well as the CCR-algebra. Nevertheless, in Section 3 we prefer to work initially with abstract $\mathrm{C}^{*}$-algebras, while in [20] concrete von Neumann algebras in a Fock representation are used. The crucial difference relies in the choice of the localisation prescription. We use $\mathcal{H}$-valued Schwartz functions on Minkowski space on which the (algebraically reducible) covariant representation $T$ of the Poincaré group acts and, in fact, we can also canonically construct the corresponding free massless quantum fields that satisfy Wightman axioms. Brunetti, Guido and Longo use the relatively recent notion of modular localisation (see also [27]) which does not need test functions on configuration space. There is also no obvious candidate for covariant representation in this frame. Recall that the covariance of free nets is expressed at the level of local reference spaces $h(\mathcal{O})$ of the CAR resp. CCR-algebras by means of the equation

$$
V(g) h(\mathcal{O})=h(g \mathcal{O}), \quad g \in \widetilde{\mathcal{P}_{+}^{\uparrow}},
$$

where $V$ is the Wigner representation (see Eq. (54)). The proof of the previous equation is based on the intertwining equation $V(g) \mathfrak{I}=\mathfrak{I} T(g)$, where $\mathfrak{I}$ is the embedding characterising the free net (for details see the proof of Theorem 3.6 in [48). The modular localisation approach uses, instead, the Bisognano-Wichmann relations as an essential input to introduce modularlike objects at the level of the one-particle Hilbert space $\mathfrak{H}$ and associated to any wedge $W$ in a suitable family of wedges $\mathcal{W}$. This family is compatible with the action of a one-parameter group of boosts and a time-reversing reflection assigned to each $W \in \mathcal{W}$. The 'Tomita operator' on $\mathfrak{H}$ naturally selects a family $K_{W}, W \in \mathcal{W}$, of $\mathbb{R}$-linear, closed, standard subspaces of $\mathfrak{H}$ that transform covariantly under the chosen Wigner representation. By means of suitable intersections of $K_{W}$ 's one defines a net of subspaces localised in e.g. causally complete convex regions which also transforms covariantly.

A remarkable aspect of the modular localisation approach is that one can also naturally associate a free net $\mathcal{O} \rightarrow \mathcal{M}_{\text {cont }}(\mathcal{O})$ to the 'continuous spin' massless representations. These types of representations are typically excluded by hand from further considerations. It is conjectured in [20, p. 761] that this net should not satisfy the Reeh-Schlieder property for double cones. If so, this would be conceptually a much more satisfactory explanation of the singular character that these representations play in nature. A natural question that arises in this context is the relation of the net $\mathcal{O} \rightarrow \mathcal{M}_{\text {cont }}(\mathcal{O})$ with the one associated to discrete helicity representations. In particular, if it is possible to describe at the $\mathrm{C}^{*}$-algebraic level in $\mathcal{M}_{\text {cont }}$ the choice of nonfaithful representation of $\mathcal{E}(2)$ needed to define discrete helicity. Techniques of local quantum constraints (see [30, 32]) may possibly be applied to $\mathcal{O} \rightarrow \mathcal{M}_{\text {cont }}(\mathcal{O})$ in order to consider this question. (Here, the use of abstract $\mathrm{C}^{*}$-algebras in a first step can be relevant.) Recall also that the use of nonfaithful representations of $\mathcal{E}(2)$ (hence discrete helicity) is crucial for the extension to the conformal group of the covariance of the corresponding net (see 1, 2, 3, 48, for further points on this subject).

Acknowledgments The present paper is a revised and considerably extended version of some parts of the authors PHD at the University of Potsdam. It is a pleasure to thank Hellmut Baumgärtel for supervision and many useful remarks. I would also like to thank Wolfgang Junker for helpful conversations.

\section{References}

[1] E. Angelopoulos and M. Flato, On the unitary implementability of conformal transformations, Lett. Math. Phys. 2 (1978), 405-412. 
[2] E. Angelopoulos, M. Flato, C. Fronsdal, and D. Sternheimer, Massless particles, conformal group and De Sitter universe, Phys. Rev. D 23 (1981), 1278-1289.

[3] E. Angelopoulos and M. Laoues, Masslessness in n-dimensions, Rev. Math. Phys. 10 (1998), 271-299.

[4] H. Araki, Indecomposable representations with invariant inner product. A theory of GuptaBleuler triplet, Commun. Math. Phys. 97 (1985), 149-159.

[5] H. Araki, Bogoljubov automorphisms and Fock representations of canonical anticommutation relations, In Operator Algebras and Mathematical Physics, (Proceedings of the summer conference held at the University of Iowa, 1985), P.E.T. Jorgensen and P.S. Muhly (eds.), American Mathematical Society, Providence, 1987.

[6] M. Asorey, L.J. Boya, and J.F. Cariñena, Covariant representations in a fibre bundle framework, Rep. Math. Phys. 21 (1985), 391-404.

[7] H. Bacry, A set of wave equations for massless fields which generalize Weyl and Maxwell equations, Il Nuovo Cimento 32 (1976), 448-460.

[8] J.C. Baez, I.E. Segal, and Z. Zhou, Introduction to Algebraic and Constructive Quantum Field Theory, Princeton University Press, Princeton, 1992.

[9] A.O. Barut and R. Rączka, Properties of non-unitary zero mass induced representations of the Poincaré group on the space of tensor-valued functions, Ann. Inst. H. Poincaré 17 (1972), 111-118.

[10] A.O. Barut and R. Rączka, Theory of Group Representations and Applications, Polish Scientific Publishers, Warszawa, 1980.

[11] H. Baumgärtel, Operatoralgebraic Methods in Quantum Field Theory. A Series of Lectures, Akademie Verlag, Berlin, 1995.

[12] H. Baumgärtel, M. Jurke, and F. Lledó, On free nets over Minkowski space, Rep. Math. Phys. 35 (1995), 101-127.

[13] H. Baumgärtel, M. Jurke, and F. Lledó, Twisted duality of the CAR-Algebra, J. Math. Phys. 43 (2002), 4158-4179.

[14] H. Baumgärtel and M. Wollenberg, Causal Nets of Operator Algebras. Mathematical Aspects of Algebraic Quantum Field Theory, Akademie Verlag, Berlin, 1992.

[15] J. Bertrand, Poincaré covariance and quantization of zero-mass fields. I. The electromagnetic field, Il Nuovo Cimento 1 A (1971), 1-12.

[16] H.J. Borchers, On the interplay between spectrum condition and locality in quantum field theory, In Operator Algebras and Mathematical Physics, (Proceedings of a summer conference held at the University of Iowa, 1985), P.E.T. Jorgensen and P.S. Muhly (eds.), American Mathematical Society, Providence, 1987.

[17] L.J. Boya, J.F. Cariñena, and M. Santander, On Proca and Wigner realizations for spin one, J. Phys. A: Math, Nucl. Gen. 7 (1974), 352-355.

[18] L.J. Boya, J.F. Cariñena, and M. Santander, On the continuity of the boosts for each orbit, Commun. Math. Phys. 37 (1974), 331-334. 
[19] R. Brunetti, D. Guido, and R. Longo, Modular structure and duality in conformal quantum field theory, Commun. Math. Phys. 156 (1993), 201-219.

[20] R. Brunetti, D. Guido, and R. Longo, Modular localization and Wigner particles, Rev. Math. Phys. 14 (2002), 759-785.

[21] D. Buchholz and R. Haag, The quest for understanding in relativistic quantum physics, J. Math. Phys. 41 (2000), 3674-3697.

[22] D. Buchholz and R. Verch, Scaling algebras and renormalization group in algebraic quantum field theory, Rev. Math. Phys. 7 (1995), 1195-1239.

[23] A. Diaz Miranda, Quantizable forms, J. Geom. Phys. 19 (1994), 47-76.

[24] A. Diaz Miranda, Wave functions in geometric quantization, Int. J. Theor. Phys. 35 (1996), 2139-2168.

[25] P.A.M. Dirac, Relativistic wave equations, Proc. Roy. Soc. London A 155 (1927), 447-459.

[26] H.P. Dürr and E. Rudolph, Indefinite metric in massless quantum field theories of arbitrary spin, Nuovo Cimento 65 A (1970), 423-466.

[27] L. Fassarella and B. Schroer, Wigner particle theory and local quantum physics, J. Phys. A 35 (2002), 9123-9164.

[28] K. Fredenhagen, On the general theory of quantized fields, In Mathematical Physics X (Proceedings, Leipzig, 1991), K. Schmüdgen (ed.), Springer Verlag, Berlin, 1992.

[29] A. Gersten, Quantum equations for massless particles of any spin, Foundations of Phys. Lett. 13 (2000), 185-192.

[30] H. Grundling and C.A. Hurst, Algebraic quantization of systems with a gauge degeneracy, Commun. Math. Phys. 98 (1985), 369-390.

[31] H. Grundling and C.A. Hurst, A note on regular states and supplementary conditions, Lett. Math. Phys. 15 (1988), 205-212, [Erratum: ibid. 17, 173-174 (1989)].

[32] H. Grundling and F. Lledó, Local quantum constraints, Rev. Math. Phys. 12 (2000), 11591218.

[33] A. Guichardet, Symmetric Hilbert Spaces and Related Topics (LNM 261), Springer Verlag, Berlin, 1972.

[34] R. Haag, Local Quantum Physics, Springer Verlag, Berlin, 1992.

[35] R. Haag and D. Kastler, An algebraic approach to quantum field theory, J. Math. Phys. 5 (1964), 848-861.

[36] D. Han and Y.S. Kim, Little group for photons and gauge transformations, Am. J. Phys. 49 (1981), 348-351.

[37] W.F. Heidenreich, On the solution space of massless field equations with arbitrary spin, J. Math. Phys. 27 (1986), 2154-2159.

[38] S. Helgason, Differential Geometry, Lie Groups, and Symmetric Spaces, Academic Press, New York, 1978. 
[39] K. Hewitt and K.A. Ross, Abstract Harmonic Analysis II, Springer Verlag, Berlin, 1994.

[40] P.D. Hislop, Conformal covariance, modular structure, and duality for local algebras in free massless quantum field theories, Ann. Phys. 185 (1988), 193-230.

[41] Y.S. Kim and E.P. Wigner, Cylindrical group and massless particles, J. Math. Phys. 28 (1987), 1175-1179.

[42] S. Kobayashi and K. Nomizu, Foundations of Differential Geometry, Interscience Publishers, New York, 1963.

[43] K. Kraus and R.F. Streater, Some covariant representations of massless Fermi fields, J. Phys. A 14 (1981), 2467-2478.

[44] S.I. Kruglov, Pair production and solutions of the wave equation for particles with arbitrary spin, Int. J. Theor. Phys. 40 (2001), 515-536.

[45] N.P. Landsman and U.A. Wiedemann, Massless particles, electromagnetism and Rieffel induction, Rev. Math. Phys. 7 (1995), 923-958.

[46] F. Lledó, A family of examples with quantum constraints, Lett. Math. Phys. 40 (1997), 223-234.

[47] F. Lledó, Algebraic properties of massless free nets, Ph.D. thesis, University of Potsdam, 1999.

[48] F. Lledó, Conformal covariance of massless free nets, Rev. Math. Phys. 13 (2001), 11351161.

[49] J.T. Łopuszański, The representations of the Poincaré group in the framework of free quantum fields, Fortschr. Phys. 28 (1978), 261-288.

[50] G.W. Mackey, The Theory of Unitary Group Representations, The University of Chicago Press, Chicago, 1976.

[51] J. Manuceau, M. Sirugue, D. Testard, and A. Verbeure, The smallest $\mathrm{C}^{*}$-algebra for canonical commutations relations, Commun. Math. Phys. 32 (1973), 231-243.

[52] M. Navarro, M. Calixto, and V. Aldaya, Relativistic field equations from higher-order polarizations of the Poincaré group, Rep. Math. Phys. 41 (1998), 193-202.

[53] U. Niederer, Massless fields as unitary representations of the Poincaré group, Fortschr. Phys. 27 (1979), 191-207.

[54] U.H. Niederer and L. O'Raifeartaigh, Realizations of the unitary representations of the inhomogeneous space-time groups II, Fortschr. Phys. 22 (1974), 131-157.

[55] R. Penrose, Zero rest-mass fields including gravitation: asymptotic behaviour, Proc. Roy. Soc. London A 284 (1965), 159-203.

[56] R. Penrose and W. Rindler, Spinors and Space-Time. Vol. 1. Two-Spinor Calculus and Relativistic Fields, Cambridge University Press, Cambridge, 1986.

[57] L. Polley, G. Reents, and R.F. Streater, Some covariant representations of massless boson fields, J. Phys. A 14 (1981), 2479-2488. 
[58] M. Reed and B. Simon, Methods of Modern Mathematical Physics II. Fourier Analysis, Self-Adjointness, Academic Press, New York, 1975.

[59] B. Schroer, The pivotal role of causality in local quantum physics, J. Phys. A, Math. Gen. 32 (1999), 5937-5974.

[60] I.E. Segal, Mathematical problems of relativistic physics, American Mathematical Society, Providence, Rhode Island, 1963.

[61] I.E. Segal, Quantization of symplectic transformations, In Mathematical Analysis and Applications, Part B, L. Nachbin (ed.), Academic Press, New York, 1981.

[62] R. Shaw, Zero-mass and nondecomposable representations, Nuovo Cimento 37 (1965), 10861099.

[63] N. Shnerb and L.P. Horwitz, Gauge and group properties of massless fields in any dimension, J. Phys. A: Math. Gen. 27 (1994), 3565-3574.

[64] D.J. Simms, Lie Groups and Quantum Mechanics, Springer Verlag, Berlin, 1968.

[65] S. Sternberg, Group Theory and Physics, Cambridge University Press, Cambridge, 1995.

[66] R.F. Streater and A.S. Wightman, PCT, Spin and Statistics, and All That, Addison-Wesley, Redwood City, 1989.

[67] F. Strocchi, Locality and covariance in QED and gravitation. General proof of Gupta-Bleuler type formulations, In Mathematical Methods in Theoretical Physics, W.E. Brittin (ed.), Colorado Ass. Univ. Press, Boulder, 1973.

[68] B. Thaller, The Dirac Equation, Springer Verlag, Berlin, 1992.

[69] V.S. Varadarajan, Geometry of Quantum Theory (2nd Ed.), Springer Verlag, Berlin, 1985.

[70] R.M. Wald, General Relativity, University of Chicago Press, Chicago, 1984.

[71] G. Warner, Harmonic Analysis on Semi-Simple Lie Groups I, Springer-Verlag, Berlin, 1972.

[72] S. Weinberg, Feynman rules for any spin. II. Massless particles, Phys. Rev. 134 (1964), B882-B896.

[73] S. Weinberg, The Quantum Theory of Fields. Vol. I, Cambridge University Press, Cambridge, 2000 .

[74] S. Weinberg, The quantum theory of massless particles, In Lectures on Particle and Field Theory (Brandais Summer Institute in Theoretical Physics 1964, vol. II ), S. Deser and K.W. Ford (eds.), Prentice Hall Inc., Englewood Cliffs, 1965.

[75] A.S. Wightman, The choice of test functions in quantum field theory, In Mathematical Analysis and Applications, Part B, L. Nachbin (ed.), Academic Press, New York, 1981.

[76] A.S. Wightman, Invariant wave equations, In Proceedings of the Ettore Majorana International School of Mathematical Physics (1977), G. Velo and A.S. Wightman (eds.), Srpinger Verlag, Berlin, 1978.

[77] E.P. Wigner, On unitary representations of the inhomogeneous Lorentz group, Ann. Math. 40 (1939), 149-204.

[78] E.P. Wigner, Relativistische Wellengleichungen, Z. Phys. 124 (1947), 665-684. 